\title{
Adiabatic regularization and Green's function of a scalar field in de Sitter space: Positive energy spectrum and no trace anomaly
}

\author{
Yang Zhang, Xuan Ye, and Bo Wang \\ Department of Astronomy, Key Laboratory for Researches in Galaxies and Cosmology, \\ School of Astronomy and Space Sciences, \\ University of Science and Technology of China, Hefei, Anhui, 230026, China
}

\begin{abstract}
In the conventional adiabatic regularization the vacuum ultraviolet divergences of a quantum field in curved spacetime are removed by subtracting the $k$-mode of the stress tensor to the 4th-order. For a scalar field in de Sitter space, we find that the 4th-order regularized spectral energy density is negative. Moreover, the 2nd-order regularization for minimal coupling $(\xi=0)$ and the 0th-order regularization for conformal coupling $\left(\xi=\frac{1}{6}\right)$ yield a positive and UV-convergent spectral energy density and power spectrum. The regularized stress tensor in the vacuum is maximally symmetric and can drive inflation, while its $k$-modes representing the primordial fluctuations are nonuniformly distributed. Conventional regularization of a Green's function in position space is generally plagued by a log IR divergence. Only in the massless case with $\xi=0$ or $\frac{1}{6}$, we can directly regularize the Green's functions and obtain vanishing results that agree with the adiabatic regularization results. In this case, the regularized power spectrum and stress tensor are both zero, and no trace anomaly exists. To overcome the log IR divergence problem in the massive cases with $\xi=0$ and $\frac{1}{6}$, we perform Fourier transformation of the regularized power spectra and obtain the regularized analytical Green's functions which are IR- and UV-convergent.
\end{abstract}

\section{inflationary universe, mathematical and relativistic aspects of cosmology, quantum fields in curved spacetimes \\ PACS numbers: $98.80 . \mathrm{Cq}, 98.80 . \mathrm{Jk}, 04.62 .+\mathrm{v}$}

\section{Introduction}

To combine quantum field theory with general relativity, a quantum field must first be place in classical curved spacetime. Quantum fluctuations in the vacuum state contain ultraviolet (UV) divergences arising from sums of the high $k$-modes $[1,2]$. These divergences manifest in physical quantities such as the stress tensor and power spectrum, and must be removed. In curved spacetime, the UV divergences cannot simply be omitted as in Minkowski spacetime, because the finite part of the fluctuations has important gravitational effects. For example, vacuum fluctuations in the perturbed inflaton field and perturbed metric were the origins of large-scale structures [3,4]. They also induced CMB anisotropies and polarization in the cosmic microwave background [5,6].

Proper removal of UV divergences is the most important priority when introducing quantum fields in classical curved spacetimes. Among the several existing regularization approaches are dimensional regularization [7-10], point-splitting [11-16], and the zeta function $[8,17]$ that can be also written as a path-integral [18]. These methods are applied to the Green's function, and the stress tensor can be constructed from derivatives of the Green's function. In these methods the

\footnotetext{
*yzh@ustc.edu.cn
} 
Green's function of a massive field is often expressed in terms of the DeWitt-Schwinger proper-time integration $[19,20]$, and they are virtually equivalent [8]. In particular, dimensional regularization is known to agree with the zeta function regularization $[8,17]$. UV divergences are isolated by expanding the Green's function in position space over a small distance $\sigma$. However, the $\ln \sigma$ term in this process is both UV- and infrared (IR)-divergent. Subtracting the $\ln \sigma$ term achieves a UVconvergent Green's function, but also introduces an IR divergence in the originally IR-convergent Green's function (as demonstrated below). This difficulty plagues all regularization methods based on the Green's function in position space. Previous researchers have selected a particular term (of 4th adiabatic order) from the series expansion of the DeWitt-Schwinger proper-time integration, and taken its massless limit $m=0$ while neglecting other terms. The result is the so-called trace anomaly $[8,9,11-13,15,17]$ of a conformally-coupling scalar field. However, the series expansion of the DeWitt-Schwinger proper-time integration is defined only at $m \neq 0$ and is actually undefined at $m=0[11,12,20]$.

The UV-divergence problem can also be solved by adiabatic regularization [21-33] which directly handles the $k$-modes of a quantum field. The spirit of this method is analogous to Einstein's equivalence principle, that is, the leading high $k$ modes regard curved spacetime as a flat spacetime. These high $k$ modes are UV divergent and should be subtracted like the UV-divergent term in quantum field theories of flat spacetime. The UV divergences of the $k$-modes next to the leading mode are subtracted by the adiabatic subtraction terms formed from the Wentzel-Kramers-Brillouin (WKB) approximations. For this purpose, a minimal subtraction rule that minimizes the adiabatic subtraction and retains the convergent terms is required. This method removes UV divergences more effectively than the Green's function-based methods; in particular, it avoids the log-term difficulty of the Green's function in position space, without causing IR divergence of a massive scalar field. In conventional adiabatic regularization, the order of the adiabatic expansion is the order of the time derivatives of the scale factor $a(\tau)$ in Robertson-Walker (RW) spacetime. Meanwhile, the minimal subtraction rule [21] commonly prescribes the 4th-order and the 2nd-order subtractions for the stress tensor and the power spectrum of a scalar field, respectively. In principle these prescriptions will sufficiently remove the UV divergences. Parker [32] explicitly obtained a 2nd-order regularized power spectrum for a minimally-coupling massive scalar field during de Sitter inflation. However, an IR- and UV-convergent regularized spectral stress tensor of a massive scalar field during de Sitter inflation remains elusive.

For a massless field, the adiabatic order matches the power of $k$, such as $\left(\frac{1}{k}\right)^{n} \rightarrow\left(a^{\prime} / a\right)^{n}$. In our previous work on relic gravitational wave (RGW) [35], we implemented adiabatic regularization in which the 4th-order regularization is both sufficient and necessary for removing the $k^{4}, k^{2}, k^{0}$ divergences from the stress tensor, whereas the 2nd-order regularization is sufficient and necessary for removing the $k^{2}, k^{0}$ divergences from the power spectrum. The slight IR distortions at $k=0$ caused by the subtraction terms can be avoided by the inside-horizon scheme [36].

The adiabatic subtraction terms of a massive field contain powers of $\omega=\left(k^{2}+m^{2} a^{2}\right)^{1 / 2}$, rather than powers of $k$. The 4th-order subtraction term for the stress tensor is constructed from time-derivatives of $a(\tau)$ up to fourth-order. Therefore, it contains powers of $\omega^{1}, \omega^{-1}, \omega^{-3}, \ldots$ $\omega^{-13}$ [28-31]. Similarly, the 2nd-order subtraction term for the power spectrum is constructed from time-derivatives of $a(\tau)$ up to second order and thus contains powers of $\omega^{-1}, \ldots \omega^{-7}[32]$. When the (positive or negative) powers of $\omega$ are further expanded to high-order terms of $k$, the subtraction terms involve infinitely many terms in powers of $k^{-1}$. Two consequences arise from this property. First, the 4th-order subtraction term will cancel not only the $k^{4}, k^{2}, k^{0}$ divergences, but also some of the convergent $k^{-2}, k^{-4}$ terms in $\left\langle T^{\mu}{ }_{\nu}\right\rangle_{k}$ for a massive field. Therefore, the regularized spectral energy density becomes negative (non-positive-definite). Second, the lower-order (0th- and 2ndorder) subtraction terms also contain $k^{4}, k^{2}, k^{0}$ terms, whose coefficients might be exactly poised to cancel the respective divergences and yield a positive, UV-convergent spectral energy density. 
Both consequences are observed in actuality. Specifically, for a minimal coupling $(\xi=0)$ massive scalar field in the vacuum state in de Sitter space, the 2nd-order adiabatic regularization yields simultaneously yields a positive UV-convergent power spectrum and a positive UV-convergent spectral energy density which respects the covariant conservation. In a conformally-coupling $\left(\xi=\frac{1}{6}\right)$ massive scalar field, the 0th-order regularization achieves a similar result. These two prescriptions achieve the proper adiabatic regularization for their respective cases. In both cases, the 4th-order regularization is an incorrect prescription, because it gives a negative spectral energy density and a negative power spectrum. For a massless scalar field with $\xi=0$ and $\xi=\frac{1}{6}$, the regularized stress tensor and power spectrum are zero, and, no conformal trace anomaly appears in the $\xi=\frac{1}{6}$ case. In the literature $[28,29]$ a trace anomaly appeared in the 4th-order adiabatic regularization of a massive scalar field with $\xi=\frac{1}{6}$ in the limit $m=0$. The present paper will demonstrate the inappropriateness of the 4th-order regularization in a scalar field with $\xi=\frac{1}{6}$. The scalar field is assumed in de Sitter space, in which the exact modes and the adiabatic subtraction terms of various orders are explicitly given. Taking the difference yields the regularized spectral stress tensor and power spectrum, respectively, in each order.

For a general coupling $\xi \in\left(0, \frac{1}{6}\right)$, we show by trial and error that no regularization of a fixed adiabatic order can simultaneously provide a positive spectral energy density and a positive power spectrum with UV convergence.

To complement the adiabatic regularization, we also regularize the Green's function in position space. We first demonstrate the $\ln \sigma$ IR divergence in a coupling massive scalar field. Only when $m=0$ and $\xi=\frac{1}{6}$ or $\xi=0$, the Green's function involves only one (or two) UV-divergent term and is regularized to zero, confirming that the adiabatic regularization yields zero regularized spectra. We also examine the literature-reported procedures for obtaining the trace anomaly by the Green's function method, and show that the result is an artifact by invalid treatments of the massless limit of the 4th-order term. Furthermore, we perform Fourier transformations on the adiabatically regularized power spectra of the massive scalar field with $\xi=0$ and $\frac{1}{6}$, and obtain the respective regularized Green's functions with IR- and UV-convergence. This regularization overcomes the log IR-divergence difficulty in the Green's function method.

The remainder of this paper is organized as follows.

Section 2 briefly introduces the exact solution of a massive scalar field during de Sitter inflation, namely, the spectral stress tensor and the power spectrum in the vacuum state.

Section 3 explores the 4th-, 2nd-, and 0th-order regularization prescriptions for $\xi=0$. Only the 2nd-order regularization is proven successful, yields a zero stress tensor and a zero power spectrum in the massless case.

Section 4 explores the 4th-, 2nd-, and 0th-order regularization prescriptions for $\xi=\frac{1}{6}$. In this case, only the 0th-order regularization works successfully, and, in the massless case, yields a zero stress tensor and a zero power spectrum with no trace anomaly.

In Sect.5, we explore the possible regularizations for a general $\xi \in\left(0, \frac{1}{6}\right)$, and show that negative spectra always occur.

Section 6 regularizes the Green's function, and pinpoints the log IR difficulty and the invalid treatments leading to the trace anomaly. In this section, we also perform Fourier transformations of the regularized power spectra for $\xi=0$ and $\frac{1}{6}$, thereby obtaining the IR- and UV-convergent Green's functions.

Section 7 discusses the results and concludes the paper.

Appendix A lists the high $k$ expansions of the exact modes. Appendix B lists the 0th-, 2nd-, and 4th-order adiabatic modes used in the context of this paper. Finally, C lists the 0th-, 2ndand 4th-order subtraction terms for the stress tensor, and demonstrates the zero four-divergence of each order. In this paper, we set $c=\hbar=1$. 


\section{The power spectrum and stress tensor of scalar field during de Sitter inflation}

The metric of a flat Robertson-Walker spacetime is

$$
d s^{2}=a^{2}(\tau)\left[d \tau^{2}-\delta_{i j} d x^{i} d x^{j}\right]
$$

with the conformal time $\tau$. The Lagrangian density of a scalar field $\phi$ is

$$
\mathcal{L}=\frac{1}{2} \sqrt{-g}\left(g^{\mu \nu} \phi_{, \mu} \phi_{, \nu}-m^{2} \phi^{2}-\xi R \phi^{2}\right)
$$

and the field equation is

$$
\left(\square+m^{2}+\xi R\right) \phi=0
$$

where $\square=\frac{1}{a^{4}} \frac{\partial}{\partial \tau}\left(a^{2} \frac{\partial}{\partial \tau}\right)-\frac{1}{a^{2}} \nabla^{2}$ is the generalized D'Alembertian operator, $m$ is the mass, $\xi$ is a coupling constant, $R=6 a^{\prime \prime} / a^{3}$ is the scalar curvature. Write

$$
\phi(\mathbf{r}, \tau)=\int \frac{d^{3} k}{(2 \pi)^{3 / 2}}\left[a_{\mathbf{k}} \phi_{k}(\tau) e^{i \mathbf{k} \cdot \mathbf{r}}+a_{\mathbf{k}}^{\dagger} \phi_{k}^{*}(\tau) e^{-i \mathbf{k} \cdot \mathbf{r}}\right]
$$

where $a_{\mathbf{k}}, a_{\mathbf{k}^{\prime}}^{\dagger}$ are the annihilation and creation operators and satisfy the canonical commutation relations. Since the field equation is linear, the $k$-modes are independent of each other. Let $\phi_{k}(\tau)=v_{k}(\tau) / a(\tau)$. The equation of $k$-mode $v_{k}$ is

$$
v_{k}^{\prime \prime}+\left(k^{2}+m^{2} a^{2}+\left(\xi-\frac{1}{6}\right) a^{2} R\right) v_{k}=0 .
$$

It should be mentioned that the conformally-coupling massless case ( $\xi=\frac{1}{6}$ and $m=0$ ) is special in that Eq.(5) reduces to $v_{k}^{\prime \prime}+k^{2} v_{k}=0$ which is a wave equation in Minkowski spacetime. We consider the de Sitter inflation with a scale factor

$$
a(\tau)=\frac{1}{H|\tau|}, \quad-\infty<\tau \leq \tau_{1},
$$

and $R=12 H^{2}$, where $H$ is a constant, and $\tau_{1}$ is the ending time of inflation. (This de Sitter inflation can be driven by several possible alternative sources, such as the cosmological constant, the quantum effective Yang-Mills condensate [37], and some type of coherent scalar inflaton field. As we shall see later in Sects. 3 and 4, it can be also driven by the regularized stress tensor of the massive scalar field $\phi$ in the vacuum.) Then

$$
v_{k}^{\prime \prime}+\left[k^{2}+\left(\frac{m^{2}}{H^{2}}+12 \xi-2\right) \tau^{-2}\right] v_{k}=0
$$

The general solution of Eq. (7) is $\propto x^{1 / 2} H_{\nu}^{(1)}(x), x^{1 / 2} H_{\nu}^{(2)}(x)$, where $H_{\nu}^{(1)}(x)=H_{\nu}^{(2) *}(x)$ are the Hankel functions, the variable $x \equiv k|\tau|$, and

$$
\nu \equiv\left(\frac{9}{4}-\frac{m^{2}}{H^{2}}-12 \xi\right)^{1 / 2}
$$

For concreteness in this paper we consider $\nu \leq 3 / 2$ and $\xi \geq 0$. The normalized solution is taken to be

$$
v_{k}(\tau) \equiv \sqrt{\frac{\pi}{2}} \sqrt{\frac{x}{2 k}} e^{i \frac{\pi}{2}\left(\nu+\frac{1}{2}\right)} H_{\nu}^{(1)}(x)
$$


which approaches the positive-frequency mode $v_{k}(\tau) \rightarrow \frac{1}{\sqrt{2 k}} e^{-i k \tau}$ in the high $k$ limit. Corresponding to this, the Bunch-Davies (BD) vacuum state is defined as the state vector $|0\rangle$ such that

$$
a_{\mathbf{k}}|0\rangle=0, \quad \text { for all } \mathbf{k}
$$

The Green's function in the BD vacuum is defined as

$$
G\left(\mathbf{r}, \mathbf{r}^{\prime}\right)=\left\langle 0\left|\phi(\mathbf{r}, \tau) \phi\left(\mathbf{r}^{\prime}, \tau\right)\right| 0\right\rangle=\frac{1}{\left|r-r^{\prime}\right|} \int_{0}^{\infty} \frac{\sin \left(k\left|r-r^{\prime}\right|\right)}{k^{2}} \Delta_{k}^{2}(\tau) d k
$$

for the equal-time $\tau=\tau^{\prime}$ case, and the auto-correlation function is

$$
\langle 0|\phi(\mathbf{r}, \tau) \phi(\mathbf{r}, \tau)| 0\rangle=\frac{1}{(2 \pi)^{3}} \int d^{3} k\left|\phi_{k}(\tau)\right|^{2}=\int_{0}^{\infty} \Delta_{k}^{2}(\tau) \frac{d k}{k},
$$

and the power spectrum associated with the Green's function is

$$
\Delta_{k}^{2}(\tau)=\frac{k^{3}}{2 \pi^{2} a^{2}}\left|v_{k}(\tau)\right|^{2}=\frac{H^{2}}{8 \pi} x^{3}\left|H_{\nu}^{(1)}(x)\right|^{2}
$$

which is nonnegative by definition. At low $k$ the power spectrum is IR convergent for the massive field. At high $k$ (i.e., $x \gg 1$ ), by (A.2), $\Delta_{k}^{2} \propto k^{3}\left(\frac{1}{2 k}+\frac{4 \nu^{2}-1}{16 k^{3} \tau^{2}}\right)+O\left(k^{-2}\right)$ leads to quadratic and logarithmic UV divergences in the $k$-integration of the auto-correlation (12). The spectrum is shown by solid line in Figure 1(a). In this paper for illustration the plots are at a time $|\tau|=1$ and with $\frac{m^{2}}{H^{2}}=0.1$ except specified otherwise. These UV divergences are to be removed by the following subtraction

$$
\Delta_{k \text { reg }}^{2}=\Delta_{k}^{2}-\Delta_{k \text { sub }}^{2}
$$

where $\Delta_{k \text { sub }}^{2}$ is a subtraction term of certain adiabatic order, formed from the WKB approximate solution. (See Appendix B.) The conventional prescription [32] adopts the 2nd-order adiabatic subtraction to the power spectrum with $\xi=0$. For other values of coupling $\xi$, nevertheless, the 2nd-order subtraction will generally lead to a negative power spectrum, as we shall see in later sections. Therefore, we shall try different order of subtraction for different $\xi$. For this purpose, we propose the following criteria for an adequate regularized power spectrum: (1) UV convergent, (2) IR convergent, (3) nonnegative.

The energy momentum tensor is given by $[8,20,28,29]$

$$
\begin{aligned}
T_{\mu \nu}= & (1-2 \xi) \partial_{\mu} \phi \partial_{\nu} \phi+\left(2 \xi-\frac{1}{2}\right) g_{\mu \nu} \partial^{\sigma} \phi \partial_{\sigma} \phi-2 \xi \phi_{; \mu \nu} \phi \\
& +\frac{1}{2} \xi g_{\mu \nu} \phi \square \phi-\xi\left(R_{\mu \nu}-\frac{1}{2} g_{\mu \nu} R+\frac{3}{2} \xi R g_{\mu \nu}\right) \phi^{2}+\left(\frac{1}{2}-\frac{3}{2} \xi\right) g_{\mu \nu} m^{2} \phi^{2}
\end{aligned}
$$

which satisfies the conservation law $T_{; \nu}^{\mu \nu}=0$ using the field equation (3). The trace is

$$
T_{\mu}^{\mu}=(6 \xi-1) \partial^{\mu} \phi \partial_{\mu} \phi+\xi(1-6 \xi) R \phi^{2}+2(1-3 \xi) m^{2} \phi^{2}
$$

and, for $\xi=\frac{1}{6}$ it reduces to

$$
T_{\mu}^{\mu}=m^{2} \phi^{2} .
$$

This expression reveals that the trace of stress tensor of the conformally-coupling massless scalar field is zero by definition. The energy density in the BD vacuum state is given by the expectation value

$$
\rho=\left\langle T_{0}^{0}\right\rangle=\int_{0}^{\infty} \rho_{k} \frac{d k}{k}
$$


where the spectral energy density is

$$
\begin{aligned}
\rho_{k} & =\frac{k^{3}}{4 \pi^{2} a^{2}}\left(\left|\phi_{k}^{\prime}\right|^{2}+k^{2}\left|\phi_{k}\right|^{2}+m^{2} a^{2}\left|\phi_{k}\right|^{2}+6 \xi \frac{a^{\prime}}{a}\left(\phi_{k}^{*} \phi_{k}^{\prime}+\phi_{k} \phi_{k}^{* \prime}\right)+6 \xi \frac{a^{\prime 2}}{a^{2}}\left|\phi_{k}\right|^{2}\right) \\
& =\frac{k^{3}}{4 \pi^{2} a^{4}}\left[\left|v_{k}^{\prime}\right|^{2}+k^{2}\left|v_{k}\right|^{2}+m^{2} a^{2}\left|v_{k}\right|^{2}+(6 \xi-1)\left(\frac{a^{\prime}}{a}\left(v_{k}^{\prime} v_{k}^{*}+v_{k} v_{k}^{* \prime}\right)-\frac{a^{\prime 2}}{a^{2}}\left|v_{k}\right|^{2}\right)\right]
\end{aligned}
$$

which is nonnegative. The pressure in the vacuum is

$$
p=-\frac{1}{3}\left\langle T^{i}{ }_{i}\right\rangle=\int_{0}^{\infty} p_{k} \frac{d k}{k}
$$

where the spectral pressure is

$$
\begin{aligned}
p_{k}= & \frac{k^{3}}{4 \pi^{2} a^{2}}\left[\left|\phi_{k}^{\prime}\right|^{2}-\frac{1}{3} k^{2}\left|\phi_{k}\right|^{2}-a^{2} m^{2}\left|\phi_{k}\right|^{2}\right. \\
& \left.+2 \xi\left(\frac{a^{\prime}}{a}\left(\phi_{k} \phi_{k}^{*^{\prime}}+\phi_{k}^{\prime} \phi_{k}^{*}\right)+\frac{a^{\prime 2}}{a^{2}}\left|\phi_{k}\right|^{2}-2\left|\phi_{k}^{\prime}\right|^{2}+2\left(k^{2}+a^{2} m^{2}\right)\left|\phi_{k}\right|^{2}+6\left(\xi-\frac{1}{6}\right) \frac{a^{\prime \prime}}{a}\left|\phi_{k}\right|^{2}\right)\right] \\
= & \frac{k^{3}}{4 \pi^{2} a^{4}}\left[\frac{1}{3}\left|v_{k}^{\prime}\right|^{2}+\frac{1}{3} k^{2}\left|v_{k}\right|^{2}-\frac{1}{3} m^{2} a^{2}\left|v_{k}\right|^{2}+2\left(\xi-\frac{1}{6}\right)\left(-2\left|v_{k}^{\prime}\right|^{2}+3 \frac{a^{\prime}}{a}\left(v_{k}^{\prime} v_{k}^{*}+v_{k} v_{k}^{* \prime}\right)\right.\right. \\
& \left.\left.-3\left(\frac{a^{\prime}}{a}\right)^{2}\left|v_{k}\right|^{2}+2\left(k^{2}+m^{2} a^{2}\right)\left|v_{k}\right|^{2}+12 \xi \frac{a^{\prime \prime}}{a}\left|v_{k}\right|^{2}\right)\right] .
\end{aligned}
$$

The spectral pressure can take both positive and negative values, unlike the spectral energy density. The trace of stress tensor is

$$
\left\langle T_{\mu}^{\mu}\right\rangle=\int\left\langle T_{\mu}^{\mu}\right\rangle_{k} \frac{d k}{k}
$$

the spectral trace is

$$
\begin{aligned}
\left\langle T_{\mu}^{\mu}\right\rangle_{k}= & \frac{k^{3}}{2 \pi^{2} a^{2}}\left[(6 \xi-1)\left(\left|\phi_{k}^{\prime}\right|^{2}-k^{2}\left|\phi_{k}\right|^{2}\right)+6 \frac{a^{\prime \prime}}{a} \xi(1-6 \xi)\left|\phi_{k}\right|^{2}+2(1-3 \xi) a^{2} m^{2}\left|\phi_{k}\right|^{2}\right] \\
= & \frac{k^{3}}{2 \pi^{2} a^{4}}\left[m^{2} a^{2}\left|v_{k}\right|^{2}+(6 \xi-1)\left(\left|v_{k}^{\prime}\right|^{2}-\frac{a^{\prime}}{a}\left(v_{k}^{\prime} v_{k}^{*}+v_{k} v_{k}^{* \prime}\right)+\frac{a^{\prime 2}}{a^{2}}\left|v_{k}\right|^{2}\right.\right. \\
& \left.\left.-\left(k^{2}+m^{2} a^{2}\right)\left|v_{k}\right|^{2}-6 \xi \frac{a^{\prime \prime}}{a}\left|v_{k}\right|^{2}\right)\right] .
\end{aligned}
$$

In the above the sums of the $k$-modes contain no product terms between different $k$ since the scalar field is linear. The stress tensor is sensitive to the coupling $\xi$. For the minimal coupling $\xi=0$,

$$
\begin{aligned}
\rho_{k} & =\frac{k^{3}}{4 \pi^{2} a^{2}}\left(\left|\left(\frac{v_{k}}{a}\right)^{\prime}\right|^{2}+k^{2}\left|\frac{v_{k}}{a}\right|^{2}+m^{2} a^{2}\left|\frac{v_{k}}{a}\right|^{2}\right) \\
& =\frac{k^{3}}{4 \pi^{2} a^{4}}\left[\left|v_{k}^{\prime}\right|^{2}-\frac{a^{\prime}}{a}\left(v_{k}^{\prime} v_{k}^{*}+v_{k} v_{k}^{* \prime}\right)+\left(\frac{a^{\prime}}{a}\right)^{2}\left|v_{k}\right|^{2}+k^{2}\left|v_{k}\right|^{2}+m^{2} a^{2}\left|v_{k}\right|^{2}\right], \\
p_{k} & =\frac{k^{3}}{4 \pi^{2} a^{2}}\left[\left|\left(\frac{v_{k}}{a}\right)^{\prime}\right|^{2}-\frac{1}{3} k^{2}\left|\frac{v_{k}}{a}\right|^{2}-m^{2} a^{2}\left|\frac{v_{k}}{a}\right|^{2}\right] \\
& =\frac{k^{3}}{4 \pi^{2} a^{4}}\left[\left|v_{k}^{\prime}\right|^{2}-\frac{a^{\prime}}{a}\left(v_{k}^{\prime} v_{k}^{*}+v_{k} v_{k}^{* \prime}\right)+\left(\frac{a^{\prime}}{a}\right)^{2}\left|v_{k}\right|^{2}-\frac{1}{3} k^{2}\left|v_{k}\right|^{2}-m^{2} a^{2}\left|v_{k}\right|^{2}\right] .
\end{aligned}
$$


For the conformal coupling $\xi=\frac{1}{6}$,

$$
\begin{aligned}
& \rho_{k}=\frac{k^{3}}{4 \pi^{2} a^{4}}\left[\left|v_{k}^{\prime}\right|^{2}+k^{2}\left|v_{k}\right|^{2}+m^{2} a^{2}\left|v_{k}\right|^{2}\right], \\
& p_{k}=\frac{k^{3}}{12 \pi^{2} a^{4}}\left[\left|v_{k}^{\prime}\right|^{2}+k^{2}\left|v_{k}\right|^{2}-m^{2} a^{2}\left|v_{k}\right|^{2}\right] .
\end{aligned}
$$

The spectral energy density and pressure are plotted in Figure 1(b) for $\xi=0$ during de Sitter inflation, $\rho_{k}$ is positive in the whole range, $p_{k}$ is positive at high $k$ and negative at low $k$ for $k|\tau|<0.82$ (outside the horizon).

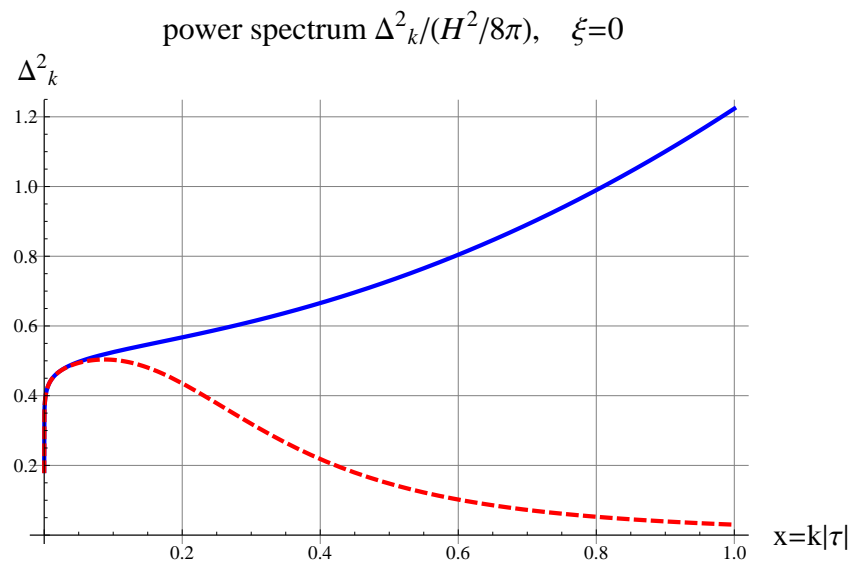

(a)

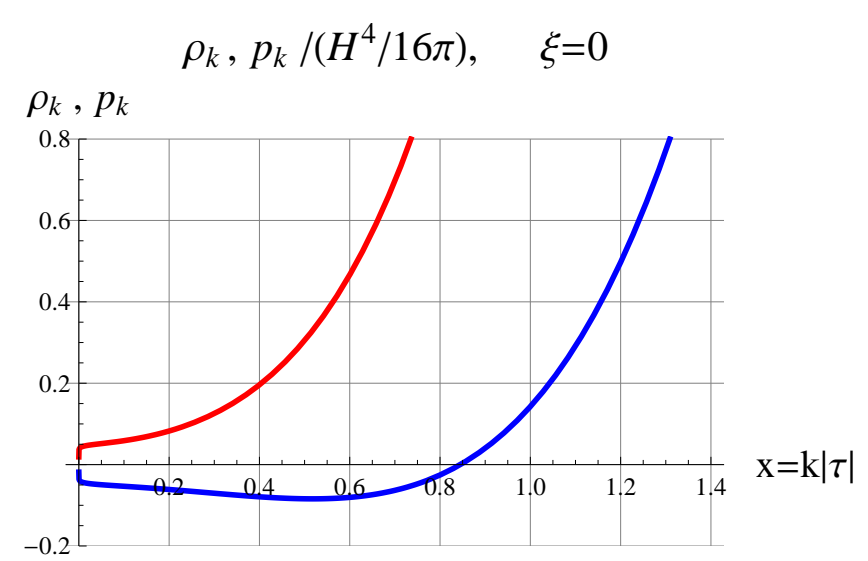

(b)

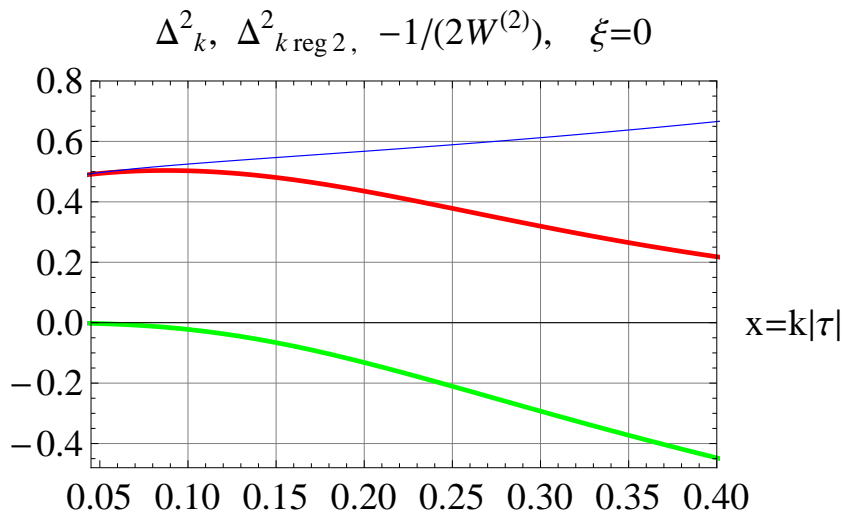

(c)

Figure 1: The plot is at a time $|\tau|=1$ in de Sitter space and for the model $\frac{m^{2}}{H^{2}}=0.1$ and $\xi=0$. (a) Blue Solid: the unregularized power spectrum $\Delta_{k}^{2}$ is IR-convergent at low $k$, and is $k^{2} \mathrm{UV}$-divergent at high $k$. Red Dash: the regularized $\Delta_{k \text { reg }}^{2}$ is $k^{-2} \mathrm{UV}$-convergent at high $k$, agreeing with that of Ref [32]. (b) Red: the unregularized spectral energy density. Blue: the unregularized spectral pressure. (c) Blue: the unregularized $\Delta_{k}^{2}$. Red: the regularized $\Delta_{k r e g}^{2}$. Green: the subtraction term, which is much lower than the unregularized.

At low $k, \rho_{k}$ and $p_{k}$ are IR convergent, dominant by the mass term. At high $k, \rho_{k}$ and $p_{k}$ contain quartic, quadratic, and logarithmic UV divergences which are to be removed by the following subtraction

$$
\left\langle T^{\mu}{ }_{\nu}\right\rangle_{k \text { reg }}=\left\langle T^{\mu}{ }_{\nu}\right\rangle_{k}-\left\langle T^{\mu}{ }_{\nu}\right\rangle_{k A}
$$

where $\left\langle T^{\mu}{ }_{\nu}\right\rangle_{k A}$ is a subtraction term constructed from the WKB solution (see Appendix C). The conventional prescription $[21,28,29]$ adopts the 4th-order adiabatic subtraction for the stress tensor. However, as we shall demonstrate in later sections, the 4th-order subtraction will generally lead 
to a negative spectral energy density. Therefore, we seek alternative prescriptions with different adiabatic orders which largely depend on the coupling $\xi$. For this, we propose the following criteria for an adequate regularized stress tensor of the scalar field: (1) UV convergent, (2) IR convergent, (3) respect the covariant conservation, (4) the spectral energy density be nonnegative. In Ref. [15] the five axioms for the regularized stress tensor did not include the positiveness of the spectral energy density. However, in frame work of general relativity, the energy of the cosmic matter must be nonnegative as a source of gravitational field. For instance, the Friedmann equation $\left(\frac{\dot{a}}{a}\right)^{2}=\frac{8 \pi G}{3} \rho$ requires that $\rho$ be nonnegative, otherwise it will lead to inconsistency. Thus we include the criterion (4). We shall show that these four criteria on the stress tensor and the three criteria on the power spectrum can be satisfied by proper regularization for $\xi=0$ and $\frac{1}{6}$ respectively.

\section{The regularization of scalar field with $\xi=0$}

We consider the massive scalar field with $\xi=0$, i.e., $\nu=\left(\frac{9}{4}-\frac{m^{2}}{H^{2}}\right)^{1 / 2}$, in de Sitter space. This important case includes a class of scalar inflaton fields in inflation models and has direct applications to cosmology of the early Universe. The power spectrum (13) is to be regularized to the adiabatic 2nd-order [32] as the following

$$
\Delta_{k \text { reg }}^{2}=\frac{k^{3}}{2 \pi^{2} a^{2}}\left(\left|v_{k}(\tau)\right|^{2}-\left|v_{k}^{(2)}(\tau)\right|^{2}\right)=\frac{k^{3}}{2 \pi^{2} a^{2}}\left(\left|v_{k}(\tau)\right|^{2}-\left(2 W_{k}^{(2)}\right)^{-1}\right)
$$

where $\left(W_{k}^{(2)}\right)^{-1}$ is given by (B.10). The resulting regularized power spectrum at $|\tau|=1$ is plotted in dashed line in Figure 1(a) for de Sitter inflation. The result agrees with that of Ref [32]. It is revealing to examine the behavior of spectrum at high $k$. By (A.2), in terms of $k$,

$$
\Delta_{k}^{2}=\frac{k^{2}}{4 \pi^{2} a^{2}}\left(1+\frac{4 \nu^{2}-1}{8 x^{2}}+\frac{3\left(4 \nu^{2}-1\right)\left(4 \nu^{2}-9\right)}{128 x^{4}}+\frac{5\left(4 \nu^{2}-1\right)\left(4 \nu^{2}-9\right)\left(4 \nu^{2}-25\right)}{1024 x^{6}}+\ldots\right),
$$

and, by (B.10) and using

$$
\begin{gathered}
\omega=\frac{1}{|\tau|}\left(x+\frac{9-4 \nu^{2}}{8 x}-\frac{\left(4 \nu^{2}-9\right)^{2}}{128 x^{3}}-\frac{\left(4 \nu^{2}-9\right)^{3}}{1024 x^{5}}-\frac{5\left(4 \nu^{2}-9\right)^{4}}{32768 x^{7}}+\ldots\right), \\
(2 \omega)^{-1} \simeq|\tau|\left(\frac{1}{2 x}+\frac{4 \nu^{2}-9}{16 x^{3}}+\frac{3\left(4 \nu^{2}-9\right)^{2}}{256 x^{5}}+\frac{5\left(4 \nu^{2}-9\right)^{3}}{2048 x^{7}}+\ldots\right),
\end{gathered}
$$

(In the following we shall set $|\tau|=1$ in the relevant expressions to avoid lengthy notations.)

$$
\left(2 W^{(2)}\right)^{-1}=\frac{1}{2 k}\left(1+\frac{4 \nu^{2}-1}{8 x^{2}}+\frac{3\left(4 \nu^{2}-1\right)\left(4 \nu^{2}-9\right)}{128 x^{4}}+\frac{5\left(4 \nu^{2}-9\right)^{2}\left(4 \nu^{2}-17\right)}{1024 x^{6}}+\ldots\right) .
$$

The first two terms in (33) just cancel the first two divergent terms of (30), so that the regularized spectrum becomes UV convergent. The third term $\frac{3\left(4 \nu^{2}-1\right)\left(4 \nu^{2}-9\right)}{128 x^{4}}$ of $(33)$ happens to cancel the convergent third term of (30), and, as a result, the regularized power spectrum is dominated by the fourth term at high $k$,

$$
\Delta_{k r e g}^{2} \simeq \frac{H^{2} x^{2}}{4 \pi^{2}} \frac{5\left(9-4 \nu^{2}\right)}{8 x^{6}}=\frac{5}{8 \pi^{2} x^{4}} \frac{m^{2}}{H^{2}} \propto k^{-4}
$$

which is positive and UV convergent. As a check, when $\Delta_{k r e g}^{2}$ is multiplied by $k^{2}$, it is found that $k^{2} \Delta_{k r e g}^{2}$ is UV convergent. This property has an important implication to a proper prescription for 
regularization of the stress tensor. Equivalently, the high $k$ behavior can be also given in terms of $\omega$, by (A.6) at $|\tau|=1$,

$$
\Delta_{k}^{2}=\frac{k^{3}}{2 \pi^{2} a^{2}}\left(\frac{1}{2 \bar{\omega}}+\frac{1}{2 \bar{\omega}^{3}}-\frac{3\left(4 \nu^{2}-9\right)}{32 \bar{\omega}^{5}}-\frac{5\left(4 \nu^{2}-9\right)\left(4 \nu^{2}+7\right)}{256 \bar{\omega}^{7}}+\frac{35\left(176 \nu^{4}-472 \nu^{2}+171\right)}{1024 \bar{\omega}^{9}}+\ldots\right)
$$

where $\bar{\omega} \equiv\left(k^{2}+\frac{m^{2}}{H^{2}}\right)^{1 / 2}$, and by $(\mathrm{B} .10)$,

$$
\left(2 W^{(2)}\right)^{-1}=\frac{1}{2 \bar{\omega}}+\frac{1}{2 \bar{\omega}^{3}}+\frac{3\left(\frac{9}{4}-\nu^{2}\right)}{8 \bar{\omega}^{5}}-\frac{5\left(\frac{9}{4}-\nu^{2}\right)^{2}}{16 \bar{\omega}^{7}}
$$

so the $\omega^{-1}, \omega^{-3}, \omega^{-5}$ terms are canceled, yielding

$$
\Delta_{k r e g}^{2} \simeq \frac{H^{2} x^{3}}{2 \pi^{2}} \frac{5\left(9-4 \nu^{2}\right)}{16 \bar{\omega}^{7}}
$$

which is equivalent to (34).

The several points should be mentioned. First, the regularized power spectrum has a factor $\left(9-4 \nu^{2}\right) \propto m^{2}$, so that it is zero at $m=0$. Second, for the massive field, $\omega=\frac{m}{H}$ at $k=0$, the subtraction term (36) based on $1 / \omega$ expansion is IR finite and smaller than $\left|v_{k}\right|^{2}$, so that regularization does not alter the convergence pattern of the power spectrum at low $k$, as shown in Figure 1(c). Third, in regard to inflation cosmology, the effects upon the power spectrum of massive field by regularization is small in low $k$ range of the observations of CMB anisotropies $\left(k\left|\tau_{1}\right| \simeq 10^{-28} \sim 10^{-25}\right)$. This is in contrast to a massless field, like RGW and scalar metric perturbations, for which the IR distortions by regularization fall into the range of cosmological observations $[35,36]$.

If we try to regularize the power spectrum for $\xi=0$ to the 0 th adiabatic order, we would get

$$
\frac{k^{3}}{2 \pi^{2} a^{2}}\left(\left|v_{k}\right|^{2}-\left|v_{k}^{(0)}\right|^{2}\right)=\frac{k^{3}}{2 \pi^{2} a^{2}}\left(\left|v_{k}\right|^{2}-\frac{1}{2 \omega}\right) \simeq \frac{H^{2} x^{3}}{2 \pi^{2}} \frac{1}{2 x^{3}}=\frac{H^{2}}{4 \pi^{2}} \propto k^{0},
$$

so that the logarithmic divergence still exists. If we try the 4 th order regularization, by (B.20) (B.22),

$$
\frac{k^{3}}{2 \pi^{2} a^{2}}\left(\left|v_{k}\right|^{2}-\left|v_{k}^{(4)}\right|^{2}\right)=\frac{k^{3}}{2 \pi^{2} a^{2}}\left(\left|v_{k}\right|^{2}-\left(2 W^{(4)}\right)^{-1}\right)
$$

the resulting spectrum will take negative values, as shown by the dashed line in Figure 2(a). This is also checked at high $k$ as follows. With $\left|v_{k}\right|^{2}$ in (A.2) and $\left(2 W^{(4)}\right)^{-1}$ in (B.20)

$$
\begin{aligned}
\left(2 W^{(4)}\right)^{-1} \simeq & \frac{1}{2 k}\left(1+\frac{4 \nu^{2}-9}{8 x^{2}}+\frac{3\left(4 \nu^{2}-9\right)\left(4 \nu^{2}-1\right)}{128 x^{4}}+\frac{5\left(4 \nu^{2}-9\right)\left(4 \nu^{2}-1\right)\left(4 \nu^{2}-25\right)}{1024 x^{6}}\right. \\
& \left.+\frac{35\left(4 \nu^{2}-9\right)^{2}\left(16 \nu^{4}-264 \nu^{2}+705\right)}{32768 x^{8}}+\ldots\right)
\end{aligned}
$$

the difference is

$$
\frac{k^{3}}{2 \pi^{2} a^{2}}\left(\left|v_{k}\right|^{2}-\left(2 W^{(4)}\right)^{-1}\right) \simeq \frac{k^{3}}{2 \pi^{2} a^{2}} \frac{1}{2 k} \frac{175\left(4 \nu^{2}-9\right)}{32 x^{8}}=-\frac{H^{2}}{2 \pi^{2}} \frac{175 \frac{m^{2}}{H^{2}}}{64 x^{6}} \propto-k^{-6}<0
$$

Not only the $k^{2}, k^{0}$ divergent terms, but also the $k^{-2}, k^{-4}$ convergent terms have been removed. Thus, the 4th order regularization of power spectrum is an incorrect prescription, as it subtracts more than necessary and violates the minimal subtraction rule. The negativeness will also occur for the 4th order regularization of stress tensor in the following. 
$\left(\Delta^{2}{ }_{k}-\Delta^{2}{ }_{k \text { A4 }}\right)$ takes negative values
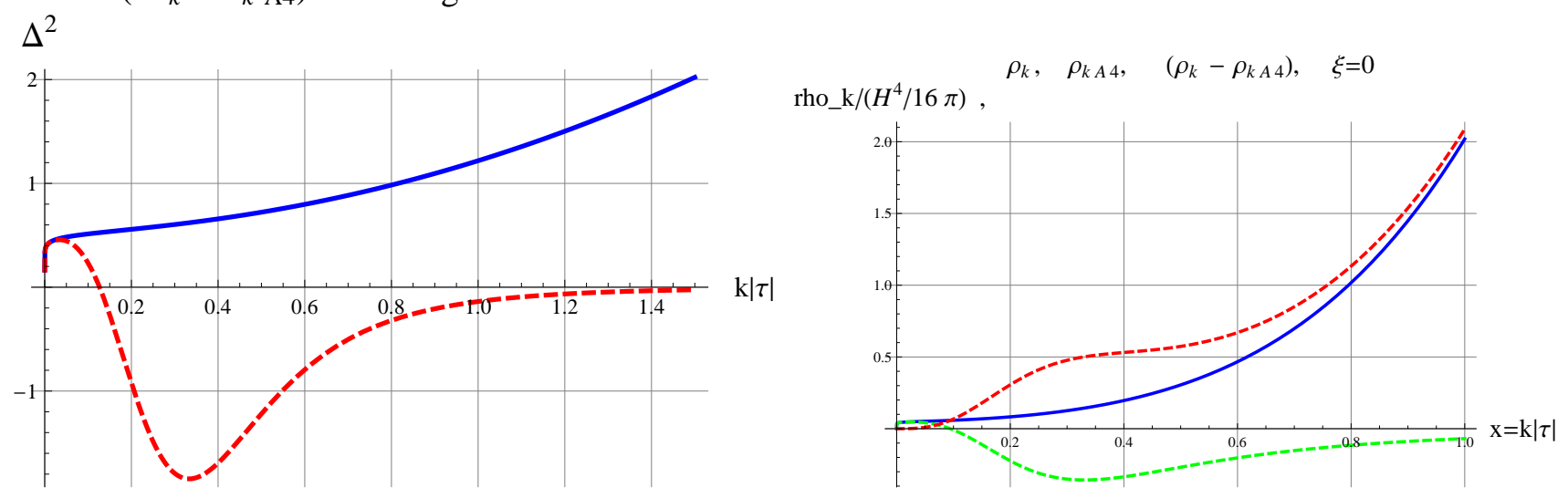

(a)

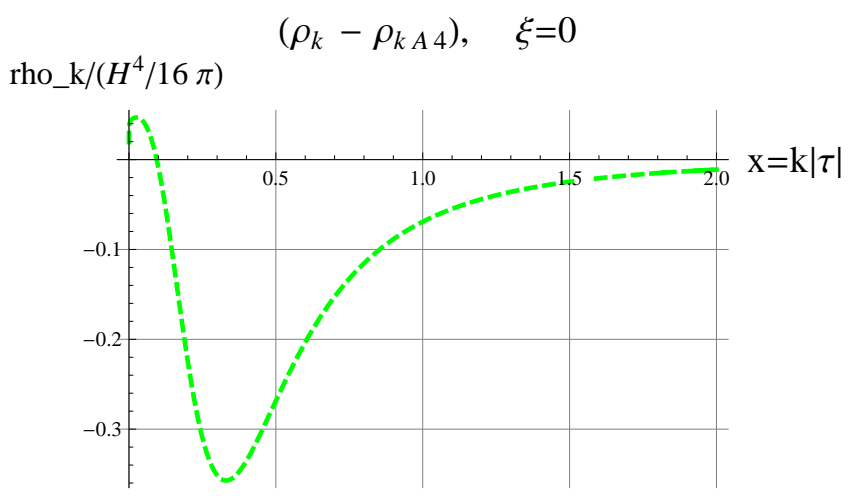

(c)

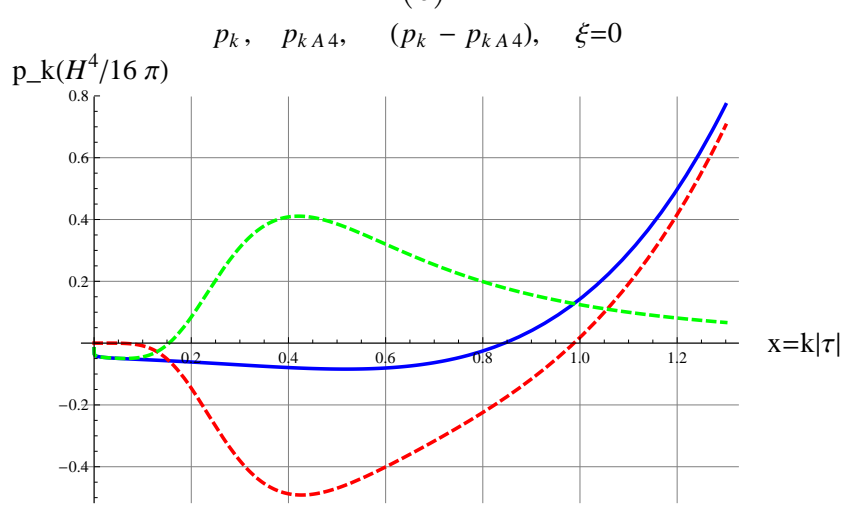

(d)

Figure 2: The model $\xi=0$. (a) Blue: the unregularized $\Delta_{k}^{2}$. Red Dash: the 4th-order regularized power spectrum is negative. (b) Blue: the unregularized $\rho_{k}$. Red: $\rho_{k A 4}$. Green: $\left(\rho_{k}-\rho_{k A 4}\right)$ which is negative for all $k|\tau| \gtrsim 0.1$ because $\rho_{k A 4}$ is higher than $\rho_{k}$. (c) $\left(\rho_{k}-\rho_{k A 4}\right)$, enlarged from (b). (d) Blue: the pressure $p_{k}$. Red: $p_{k A 4}$. Green: $\left(p_{k}-p_{k A 4}\right)$.

Now consider the adiabatic regularization of spectral energy density and spectral pressure. First we try the 4th-order regularization. The unregularized $\rho_{k}$ for $\xi=0$ during de Sitter inflation and its asymptotic high- $k$ and high- $\bar{\omega}$ expansions are the following

$$
\begin{aligned}
\rho_{k}= & \frac{H^{4}}{16 \pi}\left(x^{2}\left|\frac{d}{d x}\left(x^{3 / 2} H_{\nu}^{(2)}(x)\right)\right|^{2}+x^{5}\left|H_{\nu}^{(2)}(x)\right|^{2}+\left(\frac{9}{4}-\nu^{2}\right) x^{3}\left|H_{\nu}^{(2)}(x)\right|^{2}\right) \\
\simeq & \frac{H^{4}}{16 \pi}\left(\frac{4 x^{4}}{\pi}+\frac{\left(13-4 \nu^{2}\right) x^{2}}{2 \pi}-\frac{\left(4 \nu^{2}-9\right)\left(4 \nu^{2}-1\right)}{32 \pi}-\frac{\left(4 \nu^{2}-9\right)\left(4 \nu^{2}-1\right)\left(4 \nu^{2}+11\right)}{256 \pi x^{2}}\right. \\
& -\frac{5\left(4 \nu^{2}-9\right)\left(4 \nu^{2}-1\right)\left(4 \nu^{2}-25\right)\left(4 \nu^{2}+47\right)}{8192 \pi x^{4}} \\
& -\frac{7\left(4 \nu^{2}-9\right)\left(4 \nu^{2}-1\right)\left(4 \nu^{2}-25\right)\left(4 \nu^{2}-49\right)\left(4 \nu^{2}+99\right)}{65536 \pi x^{6}} \\
& \left.-\frac{21\left(4 \nu^{2}-9\right)\left(4 \nu^{2}-1\right)\left(4 \nu^{2}-25\right)\left(4 \nu^{2}-49\right)\left(4 \nu^{2}-81\right)\left(4 \nu^{2}+167\right)}{1048576 \pi x^{8}}+\ldots\right) \\
\simeq & \frac{H^{4}}{16 \pi} \frac{4 x^{3}}{\pi}\left[\bar{\omega}+\frac{1}{2 \bar{\omega}}+\frac{\frac{9}{4}-\nu^{2}}{2 \bar{\omega}^{3}}-\frac{\left(\frac{9}{4}-\nu^{2}\right)\left(4 \nu^{2}-29\right)}{32 \bar{\omega}^{5}}-\frac{5\left(\frac{9}{4}-\nu^{2}\right)\left(76 \nu^{2}-59\right)}{128 \bar{\omega}^{7}}\right.
\end{aligned}
$$




$$
\begin{aligned}
& -\frac{7\left(\frac{9}{4}-\nu^{2}\right)\left(32 \nu^{4}-1108 \nu^{2}+171\right)}{256 \bar{\omega}^{9}} \\
& \left.+\frac{21\left(\frac{9}{4}-\nu^{2}\right)\left(320 \nu^{6}+44176 \nu^{4}-366724 \nu^{2}+90963\right)}{32768 \bar{\omega}^{11}}+\ldots\right] .
\end{aligned}
$$

The first term, either in terms of $k$ or $\bar{\omega}$, is quartic divergent, analogous to that in the Minkowski spacetime. The first three terms, either in terms of $\bar{\omega}$ or in terms of $k$, are divergent, and the remaining are convergent. The subtraction term for spectral energy density with general $m$ and $\xi$ is listed in (C.6) in Appendix C, and, for the de Sitter inflation and $\xi=0$, it is

$$
\begin{aligned}
\rho_{k A 4}= & \frac{H^{4}}{16 \pi} \frac{4 x^{3}}{\pi}\left[\bar{\omega}+\frac{1}{2 \bar{\omega}}+\frac{\frac{9}{4}-\nu^{2}}{2 \bar{\omega}^{3}}+\frac{\left(\frac{9}{4}-\nu^{2}\right)\left(\frac{29}{4}-\nu^{2}\right)}{8 \bar{\omega}^{5}}+\frac{95\left(\frac{9}{4}-\nu^{2}\right)^{2}}{32 \bar{\omega}^{7}}\right. \\
& \left.\quad-\frac{7\left(\frac{9}{4}-\nu^{2}\right)^{3}}{8 \bar{\omega}^{9}}-\frac{105\left(\frac{9}{4}-\nu^{2}\right)^{4}}{128 \bar{\omega}^{11}}\right] \\
\simeq & \frac{H^{4}}{16 \pi}\left(\frac{4 x^{4}}{\pi}+\frac{\left(13-4 \nu^{2}\right) x^{2}}{2 \pi}-\frac{\left(4 \nu^{2}-9\right)\left(4 \nu^{2}-1\right)}{32 \pi}-\frac{\left(4 \nu^{2}-9\right)\left(4 \nu^{2}-1\right)\left(4 \nu^{2}+11\right)}{256 \pi x^{2}}\right. \\
& -\frac{5\left(4 \nu^{2}-9\right)^{2}\left(16 \nu^{4}+120 \nu^{2}-927\right)}{8192 \pi x^{4}}-\frac{7\left(4 \nu^{2}-9\right)^{3}\left(16 \nu^{4}+168 \nu^{2}-5451\right)}{65536 \pi x^{6}} \\
& \left.-\frac{21\left(4 \nu^{2}-9\right)^{4}\left(16 \nu^{4}+152 \nu^{2}-17895\right)}{1048576 \pi x^{8}}+\ldots\right) .
\end{aligned}
$$

The 4th-order regularized spectral energy density

$$
\rho_{k}-\rho_{k A 4}
$$

is negative at high $k$ as shown in Figure 2(b) and Figure 2(c) for $\frac{m^{2}}{H^{2}}=0.1$. We analytically check this at high $k$ as the following. By comparing (41) and (42), the first four terms of $\rho_{k}$ are canceled by $\rho_{k A 4}$, including its convergent $\bar{\omega}^{-5}$ term, and the remaining is dominated by the $\bar{\omega}^{-7}$ term,

$$
\rho_{k}-\rho_{k A 4} \simeq \frac{H^{4}}{16 \pi} \frac{4 x^{3}}{\pi} \frac{35\left(4 \nu^{2}-9\right)}{32 \bar{\omega}^{7}}<0
$$

which is negative. Or, equivalently, comparing (40) and (43) in terms of $k$, the first four terms $\left(\propto x^{4}, x^{2}, x^{0}, x^{-2}\right)$ of $\rho_{k}$ are canceled, leaving

$$
\rho_{k}-\rho_{k A 4} \simeq \frac{H^{4}}{16 \pi} \frac{35\left(4 \nu^{2}-9\right)}{8 \pi x^{4}}=-\frac{H^{4}}{16 \pi} \frac{35 \frac{m^{2}}{H^{2}}}{2 \pi x^{4}} \propto-k^{-4}<0
$$

which is negative, and equal to (45) up to the order $k^{-4}$. This negativeness is a difficulty of the conventional 4th-order regularization of spectral energy density for a massive scalar field. By the 4th-order prescription (42), the subtraction term $\rho_{k A 4}$ contains the terms from $\omega$ up to $\omega^{-11}$, which are more than necessary for removing the UV divergences. This is also seen in the $k$ expansion of $\rho_{k A 4}$ of (43), among which the $k^{4}, k^{2}, k^{0}$ terms are sufficient for removing the UV divergences. So the 4th-order subtraction removes too much and violates the minimal subtraction rule [21], causing a negative regularized spectral energy density.

We also give the 4th-order regularization of the spectral pressure for $\xi=0$,

$$
p_{k}=\frac{H^{4}}{16 \pi}\left(x^{2}\left|\frac{d}{d x}\left(x^{3 / 2} H_{\nu}^{(2)}(x)\right)\right|^{2}-\frac{1}{3} x^{5}\left|H_{\nu}^{(2)}(x)\right|^{2}-\left(\frac{9}{4}-\nu^{2}\right) x^{3}\left|H_{\nu}^{(2)}(x)\right|^{2}\right)
$$




$$
\begin{aligned}
= & \frac{H^{4}}{16 \pi}\left(\frac{4 x^{4}}{3 \pi}+\frac{\left(4 \nu^{2}-13\right) x^{2}}{6 \pi}+\frac{\left(4 \nu^{2}-9\right)\left(4 \nu^{2}-1\right)}{32 \pi}+\frac{5\left(4 \nu^{2}-9\right)\left(4 \nu^{2}-1\right)\left(4 \nu^{2}+11\right)}{768 \pi x^{2}}\right. \\
& +\frac{35\left(4 \nu^{2}-9\right)\left(4 \nu^{2}-1\right)\left(4 \nu^{2}-25\right)\left(4 \nu^{2}+47\right)}{24576 \pi x^{4}} \\
& +\frac{21\left(4 \nu^{2}-9\right)\left(4 \nu^{2}-1\right)\left(4 \nu^{2}-25\right)\left(4 \nu^{2}-49\right)\left(4 \nu^{2}+99\right)}{65536 \pi x^{6}} \\
& +\frac{77\left(4 \nu^{2}-9\right)\left(4 \nu^{2}-1\right)\left(4 \nu^{2}-25\right)\left(4 \nu^{2}-49\right)\left(4 \nu^{2}-81\right)\left(4 \nu^{2}+167\right)}{1048576 \pi x^{8}} \\
& \left.+\frac{143\left(4 \nu^{2}-1\right)\left(4 \nu^{2}-9\right)\left(4 \nu^{2}-25\right)\left(4 \nu^{2}-49\right)\left(4 \nu^{2}-81\right)\left(4 \nu^{2}-121\right)\left(4 \nu^{2}+251\right)}{8388608 \pi x^{10}}+\ldots\right)(47) \\
= & \frac{H^{4}}{16 \pi} \frac{4 x^{3}}{\pi} \frac{1}{3}\left[\bar{\omega}+\frac{\nu^{2}-\frac{11}{4}}{\bar{\omega}}+\frac{\nu^{2}-\frac{9}{4}}{\bar{\omega}^{3}}+\frac{\left(\nu^{2}-\frac{9}{4}\right)\left(28 \nu^{2}+37\right)}{32 \bar{\omega}^{5}}-\frac{5\left(4 \nu^{2}-9\right)\left(4 \nu^{4}+95 \nu^{2}-38\right)}{128 \bar{\omega}^{7}}\right. \\
& -\frac{7\left(4 \nu^{2}-9\right)\left(2096 \nu^{4}-24544 \nu^{2}+5733\right)}{2048 \bar{\omega}^{9}} \\
& +\frac{21\left(4 \nu^{2}-9\right)\left(448 \nu^{6}+599216 \nu^{4}-4289708 \nu^{2}+1037529\right)}{32768 \bar{\omega}^{11}} \\
& \left.+\frac{33\left(4 \nu^{2}-9\right)\left(8960 \nu^{8}+2471424 \nu^{6}-88933920 \nu^{4}+480721088 \nu^{2}-114648777\right)}{131072 \bar{\omega}^{13}}+\ldots\right],
\end{aligned}
$$

and by (C.7) the 4th-order subtraction term for pressure is

$$
\begin{aligned}
p_{k A 4}= & \frac{H^{4}}{16 \pi} \frac{4 x^{3}}{\pi} \frac{1}{3}\left[\bar{\omega}+\frac{4 \nu^{2}-11}{4 \bar{\omega}}+\frac{4 \nu^{2}-9}{4 \bar{\omega}^{3}}-\frac{-112 \nu^{4}+104 \nu^{2}+333}{128 \bar{\omega}^{5}}\right. \\
& \left.-\frac{5\left(9-4 \nu^{2}\right)^{2}\left(\nu^{2}+26\right)}{128 \bar{\omega}^{7}}-\frac{917\left(4 \nu^{2}-9\right)^{3}}{2048 \bar{\omega}^{9}}+\frac{147\left(9-4 \nu^{2}\right)^{4}}{32768 \bar{\omega}^{11}}+\frac{1155\left(4 \nu^{2}-9\right)^{5}}{131072 \bar{\omega}^{13}}\right] \\
= & \frac{H^{4}}{16 \pi}\left(\frac{4 x^{4}}{3 \pi}+\frac{\left(4 \nu^{2}-13\right) x^{2}}{6 \pi}+\frac{\left(4 \nu^{2}-9\right)\left(4 \nu^{2}-1\right)}{32 \pi}+\frac{5\left(4 \nu^{2}-1\right)\left(4 \nu^{2}-9\right)\left(4 \nu^{2}+11\right)}{768 \pi x^{2}}\right. \\
& +\frac{35\left(4 \nu^{2}-9\right)^{2}\left(16 \nu^{4}+120 \nu^{2}-927\right)}{24576 \pi x^{4}}+\frac{21\left(4 \nu^{2}-9\right)^{3}\left(16 \nu^{4}+168 \nu^{2}-5451\right)}{65536 \pi x^{6}} \\
& \left.+\frac{77\left(4 \nu^{2}-9\right)^{4}\left(16 \nu^{4}+152 \nu^{2}-17895\right)}{1048576 \pi x^{8}}+\ldots\right),
\end{aligned}
$$

so the 4th-order regularized spectral pressure for $\xi=0$ is given by

$$
p_{k}-p_{k A 4} \simeq \frac{H^{4}}{16 \pi} \frac{4 x^{3}}{\pi} \frac{-245\left(4 \nu^{2}-9\right)}{96 \omega^{7}} \propto k^{-4}
$$

which is positive except at $k|\tau| \gtrsim 0.15$, as shown in Figure 2(d). A negative, or positive spectral pressure is allowed, from physics point of view. Thus, we mainly focus on the negativeness of spectral energy density in this paper.

To avoid the difficulty of the negative spectral energy density, according to the minimal subtraction rule [21], we propose the 2 nd-order regularization for the stress tensor with $\xi=0$, i.e., the same adiabatic order as we have done upon the power spectrum. The 2nd-order subtraction term 
by (C.4) for $\xi=0$ is expressed in terms of $\bar{\omega}$, and in terms of $k$ as the following

$$
\begin{aligned}
\rho_{k A 2} & \simeq \frac{H^{4}}{16 \pi} \frac{4 x^{3}}{\pi}\left(\bar{\omega}+\frac{1}{2 \bar{\omega}}+\frac{\frac{9}{4}-\nu^{2}}{2 \bar{\omega}^{3}}+\frac{\left(\frac{9}{4}-\nu^{2}\right)^{2}}{8 \bar{\omega}^{5}}\right) \\
& \simeq \frac{H^{4}}{16 \pi}\left(\frac{4 x^{4}}{\pi}+\frac{\left(13-4 \nu^{2}\right) x^{2}}{2 \pi}-\frac{\left(4 \nu^{2}-9\right)\left(4 \nu^{2}-1\right)}{32 \pi}-\frac{\left(4 \nu^{2}-9\right)^{2}\left(4 \nu^{2}+19\right)}{256 \pi x^{2}}+\ldots\right),
\end{aligned}
$$

the 2nd-order regularized spectral energy density is

$$
\rho_{k r e g}=\rho_{k}-\rho_{k A 2}
$$

The result is positive and UV convergent, as shown in Figure 3(a) and Figure 3(b), in contrast to the negative result by the 4th-order. It is amazing that although $\rho_{k A 2}$ is defined with only two time derivatives of $a(\tau)$, it is actually sufficient to cancel all the quartic, quadratic, and logarithmic divergences in $\rho_{k}$. We analyze how this happens at high $k$. Comparing (41) and (52) in $\bar{\omega}$ expansion, the first three $\bar{\omega}, \bar{\omega}^{-1}, \bar{\omega}^{-3}$ divergent terms of $\rho_{k}$ are canceled, nevertheless, the convergent $\bar{\omega}^{-5}$ term remains. By inspection of (42) and (52), the $\bar{\omega}^{-5}$ term of $\rho_{k A 4}$ has canceled that same term of $\rho_{k}$, but the $\bar{\omega}^{-5}$ term of $\rho_{k A 2}$ does not canceled that of $\rho_{k}$. This leads to a big difference between the 2nd- and 4th-order regularization. Equivalently, comparing (40) and (53) in $k$ expansion, the first three $x^{4}, x^{2}, x^{0}$ divergent terms of $\rho_{k}$ are canceled by $\rho_{k A 2}$, but the $x^{-2}$ convergent term remains, unlike what happens with $\rho_{k A 4}$. Again this is because the $x^{-2}$ term of $\rho_{k A 2}$ has a different coefficient from that of $\rho_{k A 4}$. Thus, 2nd-order regularized spectral energy density at high $k$,

$$
\rho_{k r e g} \simeq \frac{H^{4}}{16 \pi} \frac{4 x^{3}}{\pi} \frac{5\left(9-4 \nu^{2}\right)}{32 \bar{\omega}^{5}} \simeq \frac{H^{4}}{16 \pi}\left(\frac{5\left(9-4 \nu^{2}\right)}{8 \pi x^{2}}\right) \propto m^{2} H^{2} k^{-2}>0,
$$

is positive and UV convergent, indeed.

The 2nd-order subtraction term (C.5) for pressure with $\xi=0$ is

$$
p_{k A 2}=\frac{H^{4}}{16 \pi} \frac{4 x^{3}}{\pi} \frac{1}{3}\left(\bar{\omega}+\frac{4 \nu^{2}-11}{4 \bar{\omega}}+\frac{4 \nu^{2}-9}{4 \bar{\omega}^{3}}+\frac{7\left(9-4 \nu^{2}\right)^{2}}{128 \bar{\omega}^{5}}-\frac{5\left(4 \nu^{2}-9\right)^{3}}{521 \bar{\omega}^{7}}\right),
$$

and the 2nd-order regularized spectral pressure is

$$
p_{k r e g}=p_{k}-p_{k A 2}
$$

which is UV convergent, but is negative for all $k$, as shown in Figure 3(c). At high $k$ it behaves as

$$
p_{k r e g} \simeq \frac{H^{4}}{16 \pi} \frac{4 x^{3}}{\pi}\left(\frac{25\left(4 \nu^{2}-9\right)}{96 \bar{\omega}^{5}}\right) \propto-m^{2} H^{2} k^{-2}<0 .
$$

A negative spectral pressure is allowed, from point of view of physics. The trace at high $k$ is

$$
\begin{aligned}
\left\langle T_{\mu}^{\mu}\right\rangle_{k} \simeq & \frac{H^{4}}{16 \pi}\left(\frac{\left(13-4 \nu^{2}\right) x^{2}}{\pi}-\frac{\left(4 \nu^{2}-9\right)\left(4 \nu^{2}-1\right)}{8 \pi}-\frac{3\left(4 \nu^{2}-9\right)\left(4 \nu^{2}-1\right)\left(4 \nu^{2}+11\right)}{128 \pi x^{2}}\right. \\
& -\frac{5\left(4 \nu^{2}-9\right)\left(4 \nu^{2}-1\right)\left(4 \nu^{2}-25\right)\left(4 \nu^{2}+47\right)}{1024 \pi x^{4}} \\
& \left.-\frac{35\left(4 \nu^{2}-9\right)\left(4 \nu^{2}-1\right)\left(4 \nu^{2}-25\right)\left(4 \nu^{2}-49\right)\left(4 \nu^{2}+99\right)}{32768 \pi x^{6}}+\ldots\right)
\end{aligned}
$$




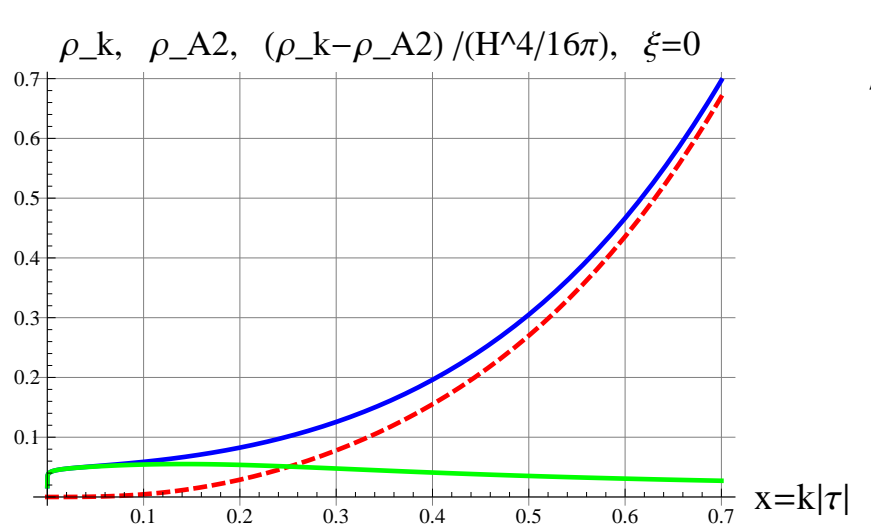

(a)

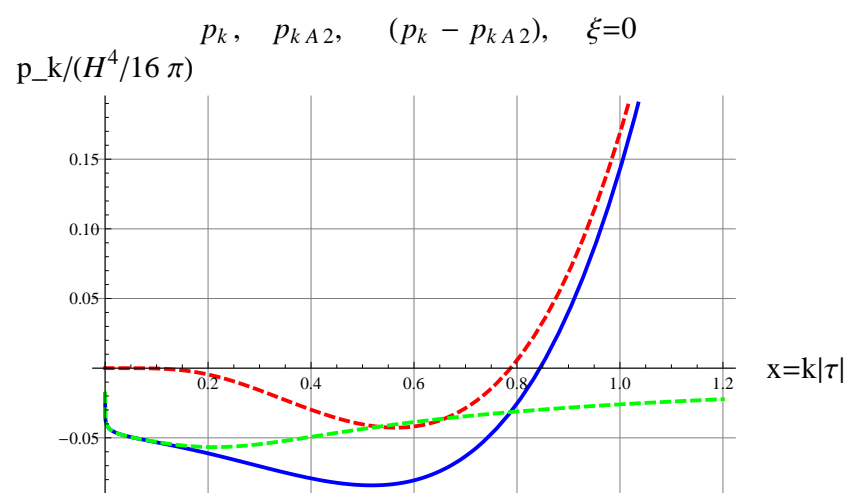

(c)

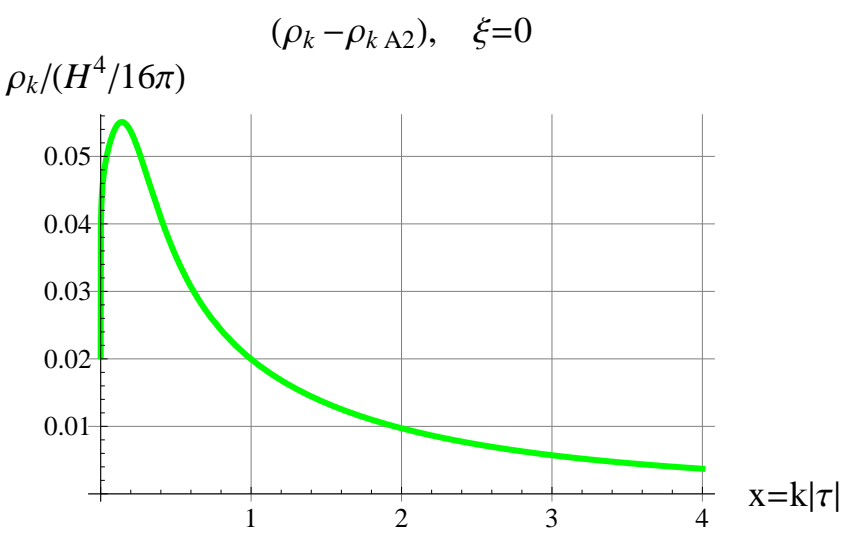

(b)

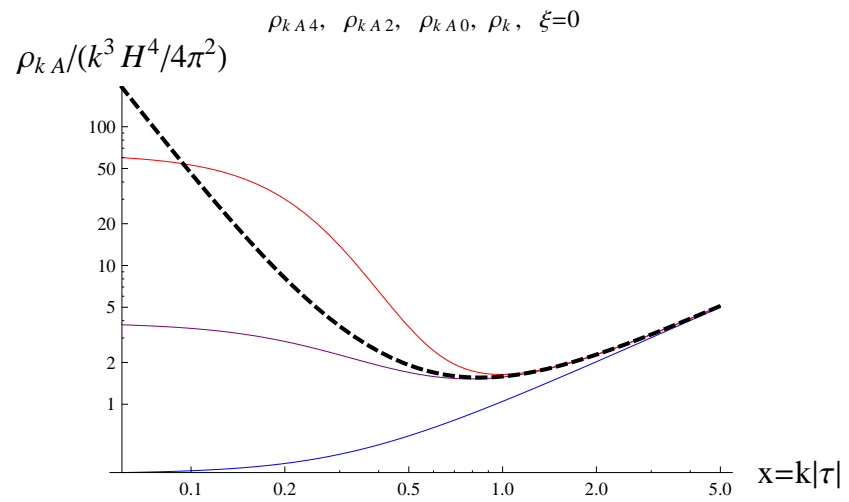

(d)

Figure 3: The model $\xi=0$. (a) Blue: the unregularized $\rho_{k}$. Red: the subtraction term $\rho_{k A 2}$. Green: the 2nd-order regularized $\left(\rho_{k}-\rho_{k A 2}\right)$. (b) $\left(\rho_{k}-\rho_{k A 2}\right)$ is positive and UV-convergent. This is enlarged from (a). (c) Blue: the unregularized $p_{k}$; Green: the subtraction term $p_{k A 2}$; Red: the regularized $\left(p_{k}-p_{k A 2}\right)$ becomes UV-convergent and is negative for all $k$. (d) The 4th (Red), 2nd (Purple), 0th (Blue) -order subtraction terms, compared with the unregularized $\rho_{k}$ (Dashed).

$$
\begin{aligned}
\simeq & \frac{H^{4}}{16 \pi} \frac{4 x^{3}}{\pi}\left[\frac{\frac{13}{4}-\nu^{2}}{\bar{\omega}}+\frac{3\left(\frac{9}{4}-\nu^{2}\right)}{2 \bar{\omega}^{3}}-\frac{3\left(4 \nu^{2}-9\right)\left(4 \nu^{2}+11\right)}{64 \bar{\omega}^{5}}\right. \\
& +\frac{5\left(4 \nu^{2}-9\right)\left(16 \nu^{4}+456 \nu^{2}-211\right)}{512 \bar{\omega}^{7}} \\
& \left.+\frac{105\left(4 \nu^{2}-9\right)\left(144 \nu^{4}-1784 \nu^{2}+405\right)}{2048 \bar{\omega}^{9}}+\ldots\right],
\end{aligned}
$$

and the 2nd-order regularized spectral trace at high $k$ is

$$
\left\langle T_{\beta}^{\beta}\right\rangle_{k r e g}=\left(\rho_{k r e g}-3 p_{k r e g}\right) \simeq \frac{H^{4}}{16 \pi} \frac{4 x^{3}}{\pi} \frac{15\left(9-4 \nu^{2}\right)}{16 \bar{\omega}^{5}} .
$$

Observe that the $k$-modes of regularized stress tensor in the vacuum state are not maximally symmetric,

$$
\left\langle T_{\mu \nu}\right\rangle_{k r e g} \neq \frac{1}{4} g_{\mu \nu}\left\langle T_{\beta}^{\beta}\right\rangle_{k r e g} .
$$

By numerical integration over $k$, the regularized energy density and pressure for the model $\frac{m^{2}}{H^{2}}=0.1$ are

$$
\rho_{\text {reg }}=\int_{0}^{\infty}\left(\rho_{k}-\rho_{k A 2}\right) \frac{d k}{k} \simeq 0.895913 \frac{H^{4}}{16 \pi}=89.5913 \frac{m^{4}}{16 \pi}>0,
$$




$$
p_{\text {reg }}=\int_{0}^{\infty}\left(p_{k}-p_{k A 2}\right) \frac{d k}{k} \simeq-0.895913 \frac{H^{4}}{16 \pi}=-89.5913 \frac{m^{4}}{16 \pi}<0,
$$

and the regularized trace is

$$
\left\langle T^{\mu}{ }_{\mu}\right\rangle_{\text {reg }}=4 \rho_{\text {reg }}=3.58365 \frac{H^{4}}{16 \pi} .
$$

Therefore, the regularized stress tensor with $\xi=0$ in the $\mathrm{BD}$ vacuum is maximally symmetric,

$$
\left\langle T_{\mu \nu}\right\rangle_{r e g}=\frac{1}{4} g_{\mu \nu}\left\langle T^{\beta}\right\rangle_{\text {reg }} .
$$

(For a model $\frac{m^{2}}{H^{2}}=1$, the numerical integrations give $\rho_{\text {reg }}=-p_{\text {reg }} \simeq 0.737829 \frac{H^{4}}{16 \pi}$, which are of the same magnitude as in the $\frac{m^{2}}{H^{2}}=0.1$ model.) (66) and (62) tells that the regularized stress tensor is maximally symmetric, but it is distributed nonuniformly in the $k$-modes. This is an interesting feature of the structure of the massive scalar field in the vacuum during de Sitter inflation. (63) and (64) give an equation of state in the vacuum $w \equiv p_{\text {reg }} / \rho_{\text {reg }}=-1$, which is the same as that of the cosmological constant. Thus, the regularized $\left\langle T_{\mu \nu}\right\rangle_{\text {reg }}$ in the vacuum state may be used as the background stress tensor that drives the de Sitter inflation. In connection with inflation cosmology, for models with $H^{2} \lesssim G^{-1}$, the regularized $\rho_{\text {reg }}$ in (63) will be smaller than the inflation energy density, $3 H^{2} /(8 \pi G)$. However, if the magnitude of $\rho_{\text {reg }}$ is comparable to the inflation energy density, it will be able to play the role to drive the inflation and the resulting inflation expansion rate will be $H \sim G^{-1 / 2}$ which is generally allowed. Thus, the massive scalar field in the vacuum can play a double role: its regularized $\left\langle T_{\mu \nu}\right\rangle_{\text {reg }}$ drives the inflation, while its $k$-modes $\phi_{k}$ together with other metric perturbations constitute the primordial fluctuations during inflation which leave imprints upon CMB anisotropies and polarization [6]. We have also checked that the four-divergence of the 2nd-order subtraction terms for the stress tensor is zero, so that the 2nd-order regularized spectral stress tensor respects the covariant conservation. (See (C.16) (C.17) in Appendix C.) Hence, the 2nd-order adiabatic regularization yields a positive, UV-convergent, covariantly-conserved spectral energy density for the minimally-coupling, massive scalar field. It is very satisfying that the same 2nd-order regularization works simultaneously for the power spectrum and the spectral stress tensor with $\xi=0$.

We also examine the 0th-order regularization. The subtraction term for spectral energy density (C.1) is

$$
\rho_{k A 0}=\frac{k^{3}}{4 \pi^{2} a^{4}} \omega \simeq \frac{H^{4}}{16 \pi}\left(\frac{4 x^{4}}{\pi}+\frac{\left(9-4 \nu^{2}\right) x^{2}}{2 \pi}-\frac{\left(\frac{9}{4}-\nu^{2}\right)^{2}}{2 \pi}+\frac{\left(\frac{9}{4}-\nu^{2}\right)^{3}}{4 \pi x^{2}}+\ldots\right)
$$

which removes only the quartic divergence of $\rho_{k}$ in (40), but the quadratic and logarithmic divergences remain. So the 0 th-order regularization fails.

For comparison, we plot the subtraction terms $\rho_{k A 0}, \rho_{k A 2}, \rho_{k A 4}$, and the unregularized $\rho_{k}$ in one graph in Figure 3(d). It reveals that $\rho_{k A 4}$ is higher than $\rho_{k}$ around $k|\tau| \gtrsim 0.1$, leading to a negative spectral energy density, $\rho_{k A 2}$ is lower than $\rho_{k}$ and gives a positive spectral energy density, $\rho_{k A 0}$ is too low.

With regard to renormalization, since the 2nd-order subtraction terms $\rho_{k A 2}$ and $p_{k A 2}$ involve only up to the second order time derivatives of metric, one does not need the fourth order time derivative counter terms ${ }^{(1)} H_{\mu \nu},{ }^{(2)} H_{\mu \nu}$ in renormalization $[1,8,28]$. The 0th-order divergent terms after $k$-integration

$$
\left\langle T^{0}{ }_{0}\right\rangle^{(0)}=\frac{1}{4 \pi^{2} a^{4}} \int_{0}^{\infty} d k k^{2} \omega, \quad\left\langle T^{1}{ }_{1}\right\rangle^{(0)}=-\frac{1}{12 \pi^{2} a^{4}} \int_{0}^{\infty} d k k^{2}\left[\omega-\frac{m^{2} a^{2}}{\omega}\right],
$$


will be absorbed by renormalizing the cosmological constant $\Lambda$, and the 2 nd-order divergent terms after the $k$ integration

$$
\begin{gathered}
\left\langle T_{0}^{0}{ }_{0}\right\rangle^{(2)}=\frac{1}{4 \pi^{2} a^{4}} \int_{0}^{\infty} d k k^{2}\left(-\frac{1}{6}\right)\left[-\frac{3}{\omega} \frac{a^{\prime 2}}{a^{2}}-\frac{3 m^{2} a^{\prime 2}}{\omega^{3}}\right], \\
\left\langle T^{1}{ }_{1}\right\rangle^{(2)}=-\frac{1}{12 \pi^{2} a^{4}} \int_{0}^{\infty} d k k^{2}\left(-\frac{1}{6}\right)\left[\frac{1}{\omega}\left(6 \frac{a^{\prime \prime}}{a}-9 \frac{a^{\prime 2}}{a^{2}}\right)+\frac{6 m^{2} a^{2}}{\omega^{3}}\left(\frac{a^{\prime \prime}}{a}-\frac{a^{\prime 2}}{a^{2}}\right)\right]
\end{gathered}
$$

will be absorbed by renormalizing the gravitation constant $G$, in the same manner as described in Refs. [1,28].

Now we study the massless minimally-coupling scalar field. The exact solution for $m=0=\xi$ is

$$
v_{k}(\tau)=-\sqrt{\frac{\pi}{2}} \sqrt{\frac{x}{2 k}} H_{\frac{3}{2}}^{(1)}(x)=-\frac{1}{\sqrt{2 k}}\left(1-\frac{i}{x}\right) e^{i x}
$$

the unregularized stress tensor (24) (25) reduces to

$$
\rho_{k}=\frac{k^{3}}{4 \pi^{2} a^{4}}\left(k+\frac{1}{2 k \tau^{2}}\right), \quad p_{k}=\frac{k^{3}}{12 \pi^{2} a^{4}}\left(k-\frac{1}{2 k \tau^{2}}\right) .
$$

The 2nd-order subtraction terms (C.4) (C.5) reduce to

$$
\rho_{k A 2}=\frac{k^{3}}{4 \pi^{2} a^{4}}\left(k+\frac{1}{2 k \tau^{2}}\right), \quad p_{k A 2}=\frac{k^{3}}{12 \pi^{2} a^{4}}\left(k-\frac{1}{2 k \tau^{2}}\right),
$$

which are just equal to the unregularized (72). Thus, we arrive at a zero regularized stress tensor

$$
\left\langle T^{\mu \nu}\right\rangle_{k r e g}=0
$$

This vanishing result also follows directly from the massless limit of (55) (58). (A zero regularized stress tensor also occurs for RGW during de Sitter inflation [35,36], whose equation is similar to a massless minimally-coupling scalar field.) The unregularized power spectrum for $m=0=\xi$ contains only two terms,

$$
\Delta_{k}^{2}=\frac{k^{3}}{2 \pi^{2} a^{2}}\left|v_{k}\right|^{2}=\frac{k^{3}}{2 \pi^{2} a^{2}} \frac{1}{2 k}\left(1+\frac{1}{x^{2}}\right)
$$

where the first term is UV divergent, and the second term is both IR and UV log divergent. Both terms are to be removed. And after adiabatic subtraction the regularized spectrum is also zero [32]

$$
\Delta_{k r e g}^{2}=\frac{k^{3}}{2 \pi^{2} a^{2}}\left(\left|v_{k}\right|^{2}-\left|v_{k}^{(2)}\right|^{2}\right)=\frac{k^{3}}{2 \pi^{2} a^{2}}\left(\frac{1}{2 k}\left(1+\frac{1}{x^{2}}\right)-\left(\frac{1}{2 k}+\frac{1}{2 k x^{2}}\right)\right)=0
$$

where $\left|v_{k}^{(2)}\right|^{2}$ is given by (B.10). The result (76) also follows from the massless limit of (34) or (37) of the massive scalar field. Hence, for the minimally-coupling massless scalar field in de Sitter space, the 2nd-order regularization yields a zero stress tensor and a zero power spectrum. We also observe that in this case the 4th-order regularization is actually equivalent to the 2nd-order one,

$$
\rho_{k A 4}=\rho_{k A 2}, \quad p_{k A 4}=p_{k A 2}
$$

as is seen from the 4th-order subtraction terms (C.6) (C.7), together with the relations (C.9) (C.10) in de Sitter space. We like to mention that the zero result (74) and (76) of the massless field may not be true in a general RW spacetime. For instance, RGW has nonzero regularized power spectrum and stress tensor during the quasi de Sitter inflation $[35,36]$. 
The lesson of the $\xi=0$ case is that, the 2 nd-order adiabatic mode $v_{k}^{(2)}$ regularizes simultaneously both the power spectrum and the stress tensor in a consistent manner. That is, when the power spectrum $\left(\propto\left|v_{k}\right|^{2}\right)$ is correctly regularized by the 2nd-order subtraction term $\left|v_{k}^{(2)}\right|^{2}$, the spectral energy density $\left(\propto\left|\left(\frac{v_{k}}{a}\right)^{\prime}\right|^{2}+\frac{k^{2}}{a^{2}}\left|v_{k}\right|^{2}+m^{2}\left|v_{k}\right|^{2}\right)$ will be regularized also correctly by the 2 nd-order subtraction term $\left|\left(\frac{v_{k}^{(2)}}{a}\right)^{\prime}\right|^{2}+\frac{k^{2}}{a^{2}}\left|v_{k}^{(2)}\right|^{2}+m^{2}\left|v_{k}^{(2)}\right|^{2}$. This outcome can be further analyzed term by term as the following. When $\left|v_{k}\right|^{2}$ is regularized correctly by $\left|v_{k}^{(2)}\right|^{2}$, giving a positive, UV convergent result, it is obvious that $m^{2}\left|v_{k}\right|^{2}$ after regularization is also positive and UV convergent. Next the $\frac{k^{2}}{a^{2}}\left|v_{k}\right|^{2}$ term which has an extra factor $k^{2}$. Since the regularized power spectrum $\propto k^{-4}$ as indicated by (34), so multiplying it by the factor $k^{2}$ will give a regularized $\frac{k^{2}}{a^{2}}\left|v_{k}\right|^{2}$ term which is still $k^{-2}$ convergent. Finally the time derivative term $\left|\left(\frac{v_{k}}{a}\right)^{\prime}\right|^{2}$. Since $v_{k} / a$ and $v_{k}^{(2)} / a$ are $\propto e^{-i k \tau}$ at high $k$, the effect of time differentiation is to bring a factor $-i k$, so that $\left(\left|\left(\frac{v_{k}}{a}\right)^{\prime}\right|^{2}-\left|\left(\frac{v_{k}^{(2)}}{a}\right)^{\prime}\right|^{2}\right) \propto$ $k^{2}\left(\left|\left(\frac{v_{k}}{a}\right)\right|^{2}-\left|\left(\frac{v_{k}^{(2)}}{a}\right)\right|^{2}\right)$, which is also $k^{-2}$ convergent.

In a general curved spacetime, the possible scheme of adiabatic regularization may be different from the 2nd order. One needs to study concretely and to see what is the appropriate scheme. As pointed out in Refs. [33,34], a prescription of adiabatic regularization may be not unique from perspective of renormalization, because the infinities to be absorbed into the bare constants can always carry along a finite term, and each different finite term will correspond to a different scheme of regularization.

\section{The regularization of scalar field with $\xi=\frac{1}{6}$}

Now we consider the massive scalar field with $\xi=\frac{1}{6}$, i.e., $\nu=\left(\frac{1}{4}-\frac{m^{2}}{H^{2}}\right)^{1 / 2}$. This is also an interesting case, and the field equation (5) in massless limit reduces to that in Minkowski spacetime. As in the last section, we also explore the 0th-, 2nd-, 4th-order regularization, respectively.

First the power spectrum. The 0th-order regularization is given by

$$
\Delta_{k r e g}^{2}=\frac{k^{3}}{2 \pi^{2} a^{2}}\left(\left|v_{k}\right|^{2}-\left|v_{k}^{(0)}\right|^{2}\right)=\frac{k^{3}}{2 \pi^{2} a^{2}}\left(\left|v_{k}\right|^{2}-\frac{1}{2 \omega}\right)
$$

The result is a positive, UV-convergent power spectrum, as shown in Figure 4(a). This can be checked at high $k$. Expanding in terms of $\bar{\omega}$, using $\left|v_{k}\right|^{2}$ of (A.7), we have

$$
\Delta_{k}^{2}=\frac{H^{2} x^{3}}{2 \pi^{2}}\left(\frac{1}{2 \bar{\omega}}+\frac{3\left(1-4 \nu^{2}\right)}{32 \bar{\omega}^{5}}+\frac{5\left(1-4 \nu^{2}\right)\left(4 \nu^{2}-25\right)}{256 \bar{\omega}^{7}}+\ldots\right) .
$$

Only the first term is UV divergent, and it is removed by the subtraction term, so the regularized power spectrum at high $\omega$ is

$$
\Delta_{k r e g}^{2} \simeq \frac{H^{2} x^{3}}{2 \pi^{2}}\left(\frac{3\left(1-4 \nu^{2}\right)}{32 \bar{\omega}^{5}}+\frac{5\left(1-4 \nu^{2}\right)\left(4 \nu^{2}-25\right)}{256 \bar{\omega}^{7}}+\ldots\right) \propto k^{-2}>0
$$

which is positive. Or, in terms of $k$, by

$$
(2 \omega)^{-1} \simeq|\tau|\left(\frac{1}{2 x}+\frac{4 \nu^{2}-1}{16 x^{3}}+\frac{3\left(4 \nu^{2}-1\right)^{2}}{256 x^{5}}+\frac{5\left(4 \nu^{2}-1\right)^{3}}{2048 x^{7}}+\ldots\right),
$$

and by (A.2), the regularized power spectrum at high $k$ is

$$
\Delta_{k r e g}^{2} \simeq \frac{H^{2} x^{3}}{2 \pi^{2}}\left(\frac{3\left(1-4 \nu^{2}\right)}{32 x^{5}}+\frac{5\left(1-4 \nu^{2}\right)\left(4 \nu^{2}-7\right)}{64 x^{7}}+\ldots\right) \propto k^{-2}>0
$$




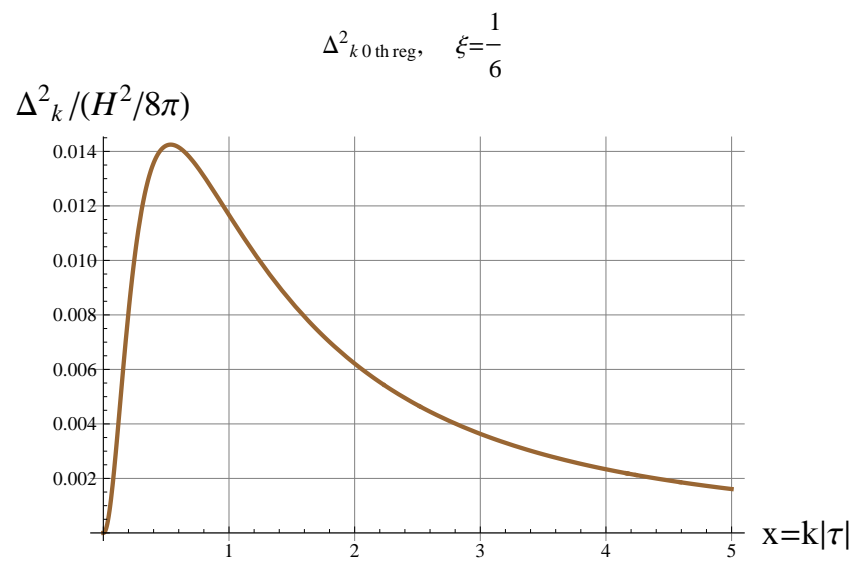

(a)

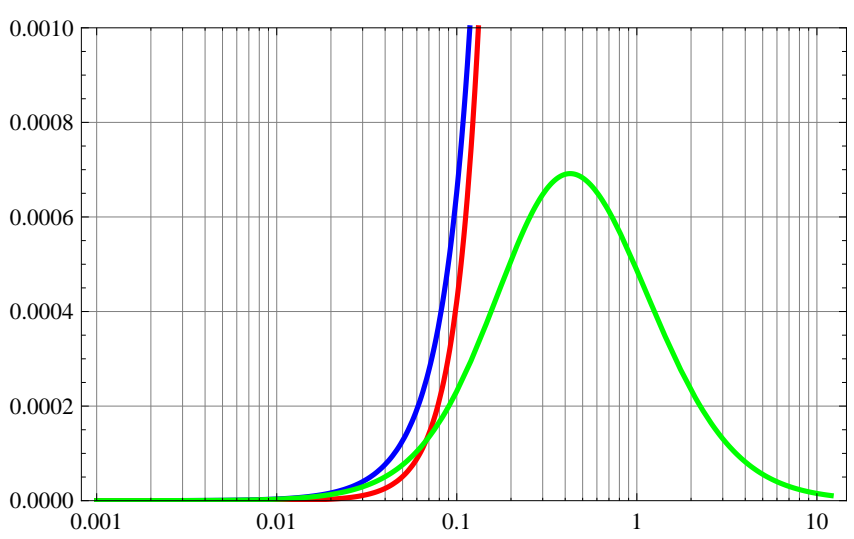

(c)

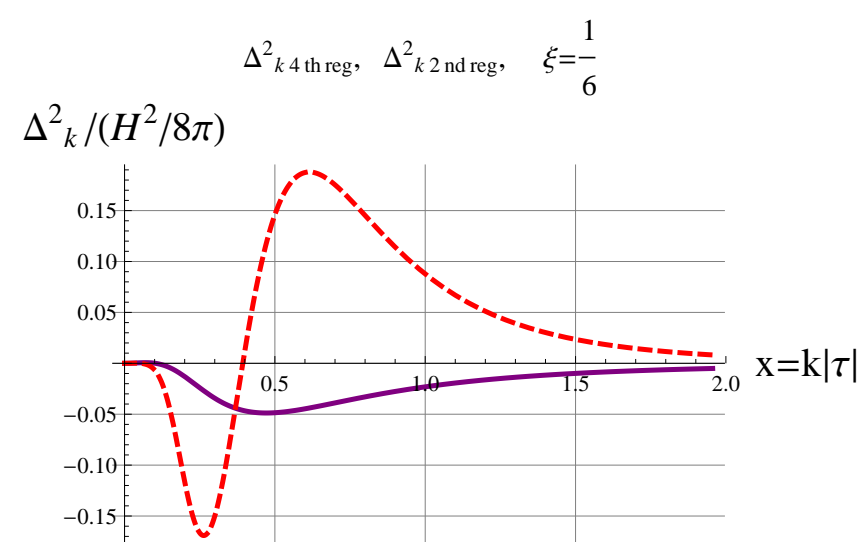

(b)

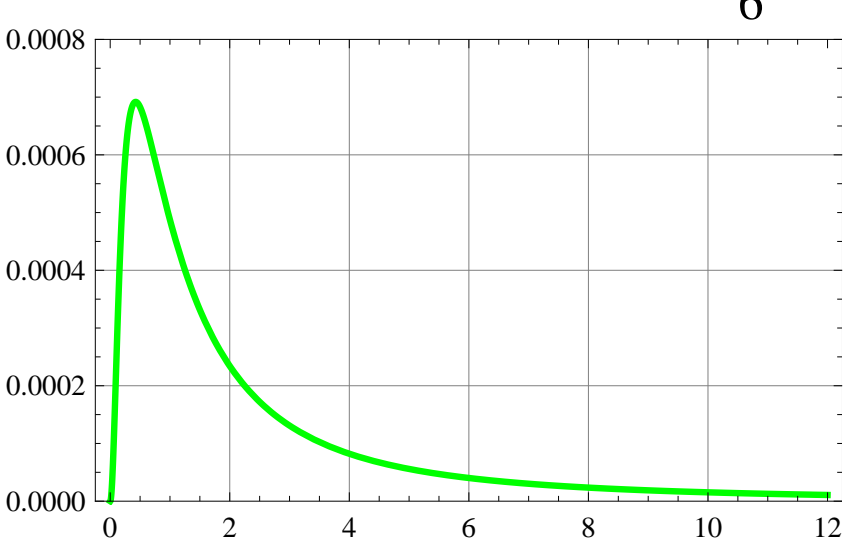

(d)

Figure 4: The model $\xi=\frac{1}{6}$. (a) The 0th-order regularized power spectrum $\Delta_{k r e g}^{2}$ is positive and UV convergent. (b) Red Dashed: the 4th-order regularized power spectrum. Purple: the 2nd-order. Both have negative values. (c) Blue: the unregularized $\rho_{k}$. Red: the subtraction term $\rho_{k}$ A0. Green: $\left(\rho_{k}-\rho_{k} A 0\right)$ is positive, IR- and UV-convergent. (d) $\left(\rho_{k}-\rho_{k A 0}\right)$, enlarged from (c).

which is equivalent to (80). The regularized power spectrum has a factor $\left(1-4 \nu^{2}\right)=4 \frac{m^{2}}{H^{2}}$, so that it will be zero for $m=0$. We also find that the 2 nd- and 4th-order regularization fail to give a positive-definite power spectrum for $\xi=\frac{1}{6}$, as shown in Figure $4(\mathrm{~b})$.

Next the spectral stress tensor. The unregularized $\rho_{k}$ at high $k$ and high- $\bar{\omega}$ is the following

$$
\begin{aligned}
\rho_{k}= & \frac{H^{4}}{16 \pi}\left(x^{4}\left|\frac{d}{d x}\left(x^{1 / 2} H_{\nu}^{(1)}(x)\right)\right|^{2}+x^{5}\left|H_{\nu}^{(1)}(x)\right|^{2}+\left(\frac{1}{4}-\nu^{2}\right) x^{3}\left|H_{\nu}^{(1)}(x)\right|^{2}\right) \\
\simeq & \frac{H^{4}}{16 \pi}\left(\frac{4 x^{4}}{\pi}+\frac{\left(1-4 \nu^{2}\right) x^{2}}{2 \pi}-\frac{\left(1-4 \nu^{2}\right)^{2}}{32 \pi}-\frac{\left(1-4 \nu^{2}\right)^{2}\left(4 \nu^{2}-9\right)}{256 \pi x^{2}}\right. \\
& -\frac{5\left(1-4 \nu^{2}\right)^{2}\left(4 \nu^{2}-9\right)\left(4 \nu^{2}-25\right)}{8192 \pi x^{4}}-\frac{7\left(1-4 \nu^{2}\right)^{2}\left(4 \nu^{2}-9\right)\left(4 \nu^{2}-25\right)\left(4 \nu^{2}-49\right)}{65536 \pi x^{6}} \\
& \left.-\frac{21\left(1-4 \nu^{2}\right)^{2}\left(4 \nu^{2}-9\right)\left(4 \nu^{2}-25\right)\left(4 \nu^{2}-49\right)\left(4 \nu^{2}-81\right)}{1048576 \pi x^{8}}+\ldots\right) \\
\simeq & \frac{H^{4}}{16 \pi} \frac{4 x^{3}}{\pi}\left[\bar{\omega}+\frac{\left(1-4 \nu^{2}\right)^{2}}{128 \bar{\omega}^{5}}-\frac{15\left(1-4 \nu^{2}\right)^{2}}{512 \bar{\omega}^{7}}-\frac{21\left(1-4 \nu^{2}\right)^{2}\left(4 \nu^{2}-13\right)}{1024 \bar{\omega}^{9}}\right.
\end{aligned}
$$




$$
\left.-\frac{105\left(1-4 \nu^{2}\right)^{2}\left(16 \nu^{4}-584 \nu^{2}+1297\right)}{32768 \bar{\omega}^{11}}+\ldots\right] .
$$

In terms of powers of $\bar{\omega},(84)$ does not contain $\bar{\omega}^{-1}$ nor $\bar{\omega}^{-3}$ terms, in contrast to (41) of $\xi=0$. The first term in (84) is quartic divergent, and the remaining terms are convergent. So, to achieve UV convergence, we need only to remove the $\bar{\omega}$ term in (84). In terms of powers of $k$, the first three terms in (83) are $k^{4}, k^{2}, k^{0}$ divergent respectively. The spectral pressure for $\xi=\frac{1}{6}$ at high $k$ and high- $\bar{\omega}$ is

$$
\begin{aligned}
p_{k}= & \frac{H^{4}}{16 \pi}\left(x^{4}\left|\frac{d}{d x}\left(x^{1 / 2} H_{\nu}^{(1)}(x)\right)\right|^{2}+x^{5}\left|H_{\nu}^{(1)}(x)\right|^{2}-\left(\frac{1}{4}-\nu^{2}\right) x^{3}\left|H_{\nu}^{(1)}(x)\right|^{2}\right) \\
\simeq & \frac{H^{4}}{16 \pi}\left(\frac{4 x^{4}}{3 \pi}+\frac{\left(4 \nu^{2}-1\right) x^{2}}{6 \pi}+\frac{\left(1-4 \nu^{2}\right)^{2}}{32 \pi}+\frac{5\left(1-4 \nu^{2}\right)^{2}\left(4 \nu^{2}-9\right)}{768 \pi x^{2}}\right. \\
& +\frac{35\left(1-4 \nu^{2}\right)^{2}\left(4 \nu^{2}-9\right)\left(4 \nu^{2}-25\right)}{24576 \pi x^{4}}+\frac{21\left(1-4 \nu^{2}\right)^{2}\left(4 \nu^{2}-9\right)\left(4 \nu^{2}-25\right)\left(4 \nu^{2}-49\right)}{65536 \pi x^{6}} \\
& +\frac{77\left(1-4 \nu^{2}\right)^{2}\left(4 \nu^{2}-9\right)\left(4 \nu^{2}-25\right)\left(4 \nu^{2}-49\right)\left(4 \nu^{2}-81\right)}{1048576 \pi x^{8}} \\
& \left.+\frac{143\left(1-4 \nu^{2}\right)^{2}\left(4 \nu^{2}-9\right)\left(4 \nu^{2}-25\right)\left(4 \nu^{2}-49\right)\left(4 \nu^{2}-81\right)\left(4 \nu^{2}-121\right)}{8388608 \pi x^{10}}+\ldots\right) \\
\simeq & \frac{H^{4}}{16 \pi} \frac{4 x^{3}\left[\frac{\bar{\omega}}{\pi}+\frac{4 \nu^{2}-1}{12 \bar{\omega}}-\frac{5\left(1-4 \nu^{2}\right)^{2}}{384 \bar{\omega}^{5}}-\frac{5\left(1-4 \nu^{2}\right)^{2}\left(2 \nu^{2}-11\right)}{768 \bar{\omega}^{7}}\right.}{}+\frac{7\left(1-4 \nu^{2}\right)^{2}\left(92 \nu^{2}-239\right)}{2048 \bar{\omega}^{9}}+\frac{7\left(1-4 \nu^{2}\right)^{2}\left(2032 \nu^{4}-36152 \nu^{2}+72271\right)}{32768 \bar{\omega}^{11}} \\
& \left.+\frac{55\left(1-4 \nu^{2}\right)^{2}\left(448 \nu^{6}-62640 \nu^{4}+602628 \nu^{2}-1045309\right)}{131072 \bar{\omega}^{13}}+\ldots\right]
\end{aligned}
$$

and the spectral trace for $\xi=\frac{1}{6}$ is

$$
\begin{aligned}
\rho_{k}-3 p_{k} \simeq & \frac{H^{4}}{16 \pi}\left(\frac{\left(1-4 \nu^{2}\right) x^{2}}{\pi}-\frac{\left(1-4 \nu^{2}\right)^{2}}{8 \pi}-\frac{3\left(1-4 \nu^{2}\right)^{2}\left(4 \nu^{2}-9\right)}{128 \pi x^{2}}\right. \\
& -\frac{5\left(1-4 \nu^{2}\right)^{2}\left(4 \nu^{2}-9\right)\left(4 \nu^{2}-25\right)}{1024 \pi x^{4}}-\frac{35\left(1-4 \nu^{2}\right)^{2}\left(4 \nu^{2}-9\right)\left(4 \nu^{2}-25\right)\left(4 \nu^{2}-49\right)}{32768 \pi x^{6}} \\
& -\frac{63\left(1-4 \nu^{2}\right)^{2}\left(4 \nu^{2}-9\right)\left(4 \nu^{2}-25\right)\left(4 \nu^{2}-49\right)\left(4 \nu^{2}-81\right)}{262144 \pi x^{8}} \\
& \left.-\frac{231\left(1-4 \nu^{2}\right)^{2}\left(4 \nu^{2}-9\right)\left(4 \nu^{2}-25\right)\left(4 \nu^{2}-49\right)\left(4 \nu^{2}-81\right)\left(4 \nu^{2}-121\right)}{4194304 \pi x^{10}}+\ldots\right) \\
\simeq & \frac{H^{4}}{16 \pi} \frac{4 x^{3}}{\pi}\left[\frac{\frac{1}{4}-\nu^{2}}{\bar{\omega}}+\frac{3\left(1-4 \nu^{2}\right)^{2}}{64 \bar{\omega}^{5}}+\frac{5\left(1-4 \nu^{2}\right)^{2}\left(4 \nu^{2}-25\right)}{512 \bar{\omega}^{7}}\right. \\
& -\frac{105\left(1-4 \nu^{2}\right)^{2}\left(20 \nu^{2}-53\right)}{2048 \bar{\omega}^{9}}-\frac{63\left(1-4 \nu^{2}\right)^{2}\left(176 \nu^{4}-3256 \nu^{2}+6563\right)}{8192 \bar{\omega}^{11}} \\
& \left.+\frac{1155\left(1-4 \nu^{2}\right)^{2}\left(64 \nu^{6}-9456 \nu^{4}+92268 \nu^{2}-160717\right)}{131072 \omega^{13}}+\ldots\right] .
\end{aligned}
$$


In the $\bar{\omega}$ expansion the spectral trace has only an $\bar{\omega}^{-1}$ divergent term, and, in the $k$ expansion, it has only $k^{2}, k^{0}$ divergences. There is a common factor $\left(1-4 \nu^{2}\right)=4 \frac{m^{2}}{H^{2}}$, so in the limit $m=0$ the trace is zero, consistent with its definition (16).

The 0th-order subtraction term for spectral energy density (C.1) is written as

$$
\begin{aligned}
\rho_{k A 0} & =\frac{H^{4}}{16 \pi} \frac{4 x^{3}}{\pi} \bar{\omega} \\
& \simeq \frac{H^{4}}{16 \pi}\left(\frac{4 x^{4}}{\pi}+\frac{\left(1-4 \nu^{2}\right) x^{2}}{2 \pi}-\frac{\left(1-4 \nu^{2}\right)^{2}}{32 \pi}+\frac{\left(1-4 \nu^{2}\right)^{3}}{256 \pi x^{2}}+\ldots\right) .
\end{aligned}
$$

In terms of $\bar{\omega}$, it cancels the only $\bar{\omega}$ divergent term of $\rho_{k}$ in $(84)$, or in terms of $k$, it cancels all the $k^{4}, k^{2}, k^{0}$ divergent terms of $\rho_{k}$ in (83). The 0th-order regularized spectral energy density

$$
\rho_{k r e g}=\rho_{k}-\rho_{k A 0}
$$

is positive, UV convergent, as shown in Figure 4(c) and Figure 4(d). At high $k$, it is dominated by the 4 th term

$$
\begin{aligned}
\rho_{k r e g} \simeq & \frac{H^{4}}{16 \pi} \frac{4 x^{3}}{\pi}\left[\frac{\left(1-4 \nu^{2}\right)^{2}}{128 \bar{\omega}^{5}}-\frac{15\left(1-4 \nu^{2}\right)^{2}}{512 \bar{\omega}^{7}}-\frac{21\left(1-4 \nu^{2}\right)^{2}\left(4 \nu^{2}-13\right)}{1024 \bar{\omega}^{9}}\right. \\
& \left.-\frac{105\left(1-4 \nu^{2}\right)^{2}\left(16 \nu^{4}-584 \nu^{2}+1297\right)}{32768 \bar{\omega}^{11}}+\ldots\right] \\
\simeq & \frac{H^{4}}{16 \pi} \frac{\left(1-4 \nu^{2}\right)^{2}}{32 \pi x^{2}} \propto k^{-2}>0
\end{aligned}
$$

which is $k^{-2}$ convergent at high $k$. For the spectral pressure, the 0th-order subtraction term is given by (C.2) and is written as

$$
p_{k A 0}=\frac{H^{4}}{16 \pi} \frac{4 x^{3}}{\pi}\left[\frac{\bar{\omega}}{3}-\frac{\left(1-4 \nu^{2}\right)}{12 \bar{\omega}}\right]
$$

and the regularized pressure is given by

$$
p_{k r e g}=p_{k}-p_{k A 0}
$$

which is negative for the whole range of $k$, as shown in Figure 5(a). This is checked at high $k$,

$$
\begin{aligned}
p_{k}-p_{k A 0} \simeq & \frac{H^{4}}{16 \pi} \frac{4 x^{3}}{\pi}\left[-\frac{5\left(1-4 \nu^{2}\right)^{2}}{384 \bar{\omega}^{5}}-\frac{5\left(1-4 \nu^{2}\right)^{2}\left(2 \nu^{2}-11\right)}{768 \bar{\omega}^{7}}+\frac{7\left(1-4 \nu^{2}\right)^{2}\left(92 \nu^{2}-239\right)}{2048 \bar{\omega}^{9}}\right. \\
& \left.+\frac{7\left(1-4 \nu^{2}\right)^{2}\left(2032 \nu^{4}-36152 \nu^{2}+72271\right)}{32768 \bar{\omega}^{11}}+\ldots\right] \\
\simeq & -\frac{H^{4}}{16 \pi} \frac{4 x^{3}}{\pi} \frac{5\left(1-4 \nu^{2}\right)^{2}}{384 \bar{\omega}^{5}}<0
\end{aligned}
$$

which is negative. Again a negative pressure is allowed. The 0th-order subtraction term for the trace is

$$
\rho_{k A 0}-3 p_{k A 0}=\frac{H^{4}}{16 \pi} \frac{4 x^{3}}{\pi} \frac{\left(1-4 \nu^{2}\right)}{4 \bar{\omega}}
$$




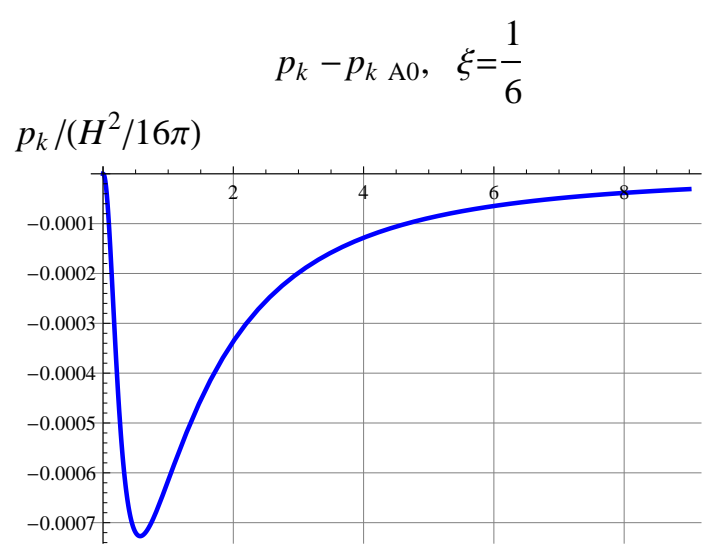

(a)

$\rho_{k}, \rho_{k A 4},\left(\rho_{k}-\rho_{k A 4}\right), \xi=\frac{1}{6}$

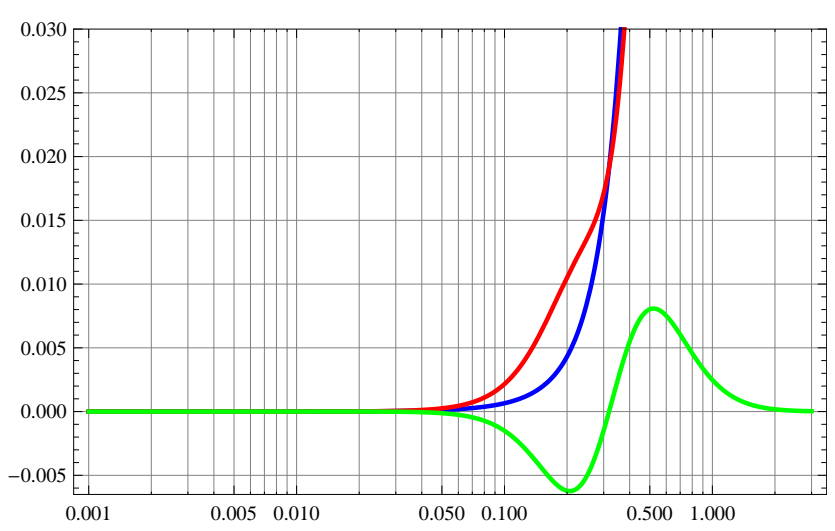

(c) $\rho_{k}, \rho_{k A 2}, \quad\left(\rho_{k}-\rho_{k A 2}\right), \quad \xi=\frac{1}{6}$

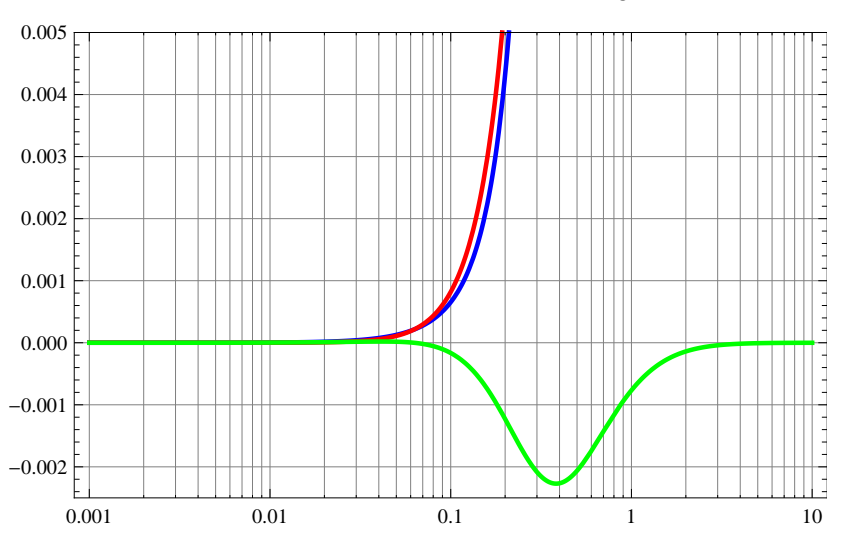

(b)

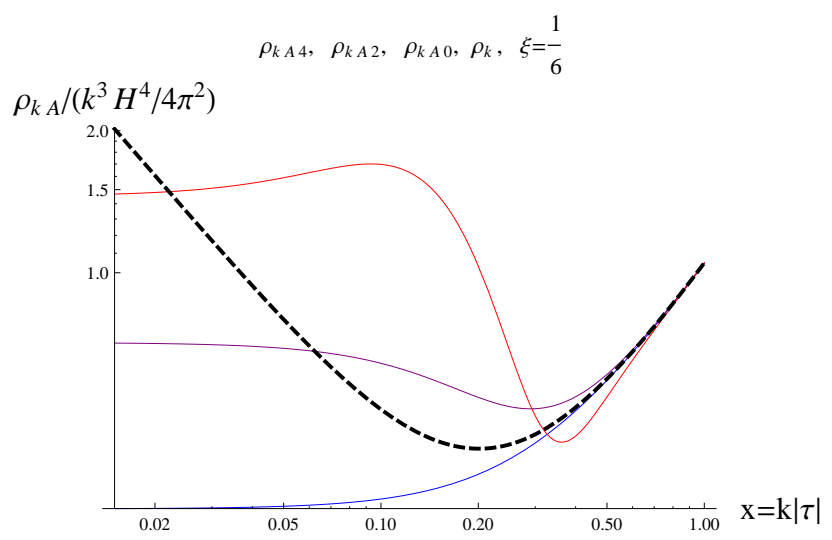

(d)

Figure 5: The model $\xi=\frac{1}{6}$. (a) The 0th-order regularized spectral pressure $\left(p_{k}-p_{k A 0}\right)$. (b) Blue: the unregularized $\rho_{k}$. Red: the subtraction term $\rho_{k A 2}$. Green: $\left(\rho_{k}-\rho_{k A 2}\right)$ is negative for all $k$. (c) Blue: the unregularized $\rho_{k}$. Red: the subtraction term $\rho_{k A 4}$ of (107). Green: $\left(\rho_{k}-\rho_{k A 4}\right)$ is negative for $k|\tau| \lesssim 0.3$. (d) The 4th (Red), 2nd (Purple), 0th (Blue) -order subtraction terms, compared with the unregularized $\rho_{k}$ (Dashed).

which is proportional to $\mathrm{m}^{2}$. The difference between (88) and (98) gives the 0th-order regularized spectral trace

$$
\left\langle T^{\mu}{ }_{\mu}\right\rangle_{k r e g} \simeq \frac{H^{4}}{16 \pi} \frac{4 x^{3}}{\pi}\left[\frac{3\left(1-4 \nu^{2}\right)^{2}}{64 \bar{\omega}^{5}}+\frac{5\left(1-4 \nu^{2}\right)^{2}\left(4 \nu^{2}-25\right)}{512 \bar{\omega}^{7}}-\frac{105\left(1-4 \nu^{2}\right)^{2}\left(20 \nu^{2}-53\right)}{2048 \bar{\omega}^{9}}+\ldots\right]
$$

which is UV convergent. The regularized trace has a factor $\left(1-4 \nu^{2}\right)^{2}=16 \frac{m^{4}}{H^{4}}$, and vanishes in the limit $m=0$, and no trace anomaly exists. There is a relation between the regularized spectral trace (99) and the regularized power spectrum (80) for $\xi=\frac{1}{6}$ as follows

$$
\left\langle T_{\beta}^{\beta}\right\rangle_{k r e g}=H^{2}\left(\frac{1}{4}-\nu^{2}\right) \Delta_{k r e g 0}^{2}=m^{2} \Delta_{k r e g 0}^{2}
$$

which, after $k$-integration, yields

$$
\left\langle T_{\beta}^{\beta}\right\rangle_{r e g}=m^{2} G(0)_{r e g}
$$


where $G(0)_{\text {reg }}$ is the regularized, finite auto-correlation function. The result (101) is consistent with the definition relation (17) for $\xi=\frac{1}{6}$. (See Figure 10(a) for the value of $G(0)_{\text {reg }}$ and Sect.6 for the Green's function.) After numerical integration over $k$, the 0th-order regularized energy density and pressure (for the model $\frac{m^{2}}{H^{2}}=0.1$ ) are the following

$$
\begin{gathered}
\rho_{\text {reg }}=\int_{0}^{\infty}\left(\rho_{k}-\rho_{k A 0}\right) \frac{d k}{k} \simeq 0.001786 \frac{H^{4}}{16 \pi}=0.1786 \frac{m^{4}}{16 \pi}>0, \\
p_{\text {reg }} \int_{0}^{\infty}\left(p_{k}-p_{k A 0}\right) \frac{d k}{k} \simeq-0.001786 \frac{H^{4}}{16 \pi}=-0.1786 \frac{m^{4}}{16 \pi}<0,
\end{gathered}
$$

and the regularized trace

$$
\left\langle T_{\mu}^{\mu}\right\rangle_{r e g}=4 \rho_{r e g}=0.007143 \frac{H^{4}}{16 \pi}=0.7143 \frac{m^{4}}{16 \pi} .
$$

So the regularized stress tensor with $\xi=\frac{1}{6}$ in the vacuum is also maximally symmetric,

$$
\left\langle T_{\mu \nu}\right\rangle_{r e g}=\frac{1}{4} g_{\mu \nu}\left\langle T_{\beta}^{\beta}\right\rangle_{r e g}
$$

similar to Eq.(66) for $\xi=0$, although it is nonuniformly distributed among the $k$-modes. In regard to the magnitude of vacuum energy density (102) for $\xi=\frac{1}{6}$ is about three orders lower than (63) for $\xi=0$. For the regularized stress tensor in the vacuum to drive inflation, one would have a very high rate expansion $H \sim 50 G^{-1 / 2}$ for a model $\frac{m^{2}}{H^{2}}=0.1$ and $\xi=\frac{1}{6}$. Its $k$-modes $\phi_{k}$ still can be part of the primordial fluctuations. As (C.12) (C.13) show, the four-divergence of the 0th-order subtraction terms for stress tensor is zero, so that the 0th-order regularized spectral stress tensor respects the covariant conservation. Hence, the 0th-order adiabatic regularization yields a positive, UV-convergent, covariantly-conserved spectral energy density for the conformally-coupling, massive scalar field. Thus, the 0th-order regularization works simultaneously for both the power spectrum and the spectral stress tensor with $\xi=\frac{1}{6}$.

We next examine what happens for the 2 nd-order regularization. From (C.4) for $\xi=\frac{1}{6}$ the 2nd-order subtraction term is

$$
\begin{aligned}
\rho_{k A 2}= & \frac{H^{4}}{16 \pi} \frac{4 x^{3}}{\pi}\left[\bar{\omega}+\frac{\left(1-4 \nu^{2}\right)^{2}}{128 \bar{\omega}^{5}}\right] \\
\simeq & \frac{H^{4}}{16 \pi}\left(\frac{4 x^{4}}{\pi}+\frac{\left(1-4 \nu^{2}\right) x^{2}}{2 \pi}-\frac{\left(1-4 \nu^{2}\right)^{2}}{32 \pi}-\frac{\left(1-4 \nu^{2}\right)^{2}\left(4 \nu^{2}-9\right)}{256 \pi x^{2}}\right. \\
& \left.\quad-\frac{5\left(4 \nu^{2}-33\right)\left(4 \nu^{2}-1\right)^{3}}{8192 \pi x^{4}}+\ldots\right) .
\end{aligned}
$$

This will subtract too much, removing not only the $\bar{\omega}$ term, but also the $\bar{\omega}^{-5}$ term of $(84)$, or in terms of $k$, removing the first four terms of $\rho_{k}$ of (83). So, for a conformally-coupling massive scalar field, the 2nd-order regularization violates the minimal subtraction rule, and is an incorrect prescription, leading to a negative spectral energy density, as shown in Figure 5(b).

We then examine the 4 th-order regularization. From (C.6) for $\xi=\frac{1}{6}$ the 4 th-order subtraction 
term is

$$
\begin{aligned}
\rho_{k A 4}= & \frac{H^{4}}{16 \pi} \frac{4 x^{3}}{\pi}\left[\bar{\omega}+\frac{\left(\frac{1}{4}-\nu^{2}\right)^{2}}{8 \bar{\omega}^{5}}-\frac{15\left(\frac{1}{4}-\nu^{2}\right)^{2}}{32 \bar{\omega}^{7}}+\frac{21\left(\frac{1}{4}-\nu^{2}\right)^{3}}{16 \bar{\omega}^{9}}-\frac{105\left(\frac{1}{4}-\nu^{2}\right)^{4}}{128 \bar{\omega}^{11}}\right] \\
\simeq & \frac{H^{4}}{16 \pi}\left(\frac{4 x^{4}}{\pi}+\frac{\left(1-4 \nu^{2}\right) x^{2}}{2 \pi}-\frac{\left(1-4 \nu^{2}\right)^{2}}{32 \pi}-\frac{\left(1-4 \nu^{2}\right)^{2}\left(4 \nu^{2}-9\right)}{256 \pi x^{2}}\right. \\
& \left.-\frac{5\left(1-4 \nu^{2}\right)^{2}\left(4 \nu^{2}-9\right)\left(4 \nu^{2}-25\right)}{8192 \pi x^{4}}-\frac{7\left(4 \nu^{2}-1\right)^{3}\left(16 \nu^{4}-328 \nu^{2}+1809\right)}{65536 \pi x^{6}}+\ldots\right) .
\end{aligned}
$$

This also subtracts off too much, canceling not only the divergent $\bar{\omega}$ term and but also the convergent $\bar{\omega}^{-5}, \bar{\omega}^{-7}$ terms in (107), thus violating the minimal subtraction rule. Or, in terms $k$, it removes not only the $k^{4}, k^{2}, k^{0}$ divergent terms but also the $k^{-2}, k^{-4}$ convergent terms of $\rho_{k}$ in (83). The resulting spectral energy density $\left(\rho_{k}-\rho_{k} A 4\right)$ is negative in the low $k$ range, as shown in Figure $5(\mathrm{c})$. Thus, the 4th-order regularization is an incorrect prescription for a conformally-coupling massive scalar field. We plot $\rho_{k A 0}, \rho_{k A 2}, \rho_{k A 4}, \rho_{k}$ together in Figure $5(\mathrm{~d})$, which reveals that only $\rho_{k A 0}$ is lower than $\rho_{k}$ and yields a positive $\rho_{k r e g}$, whereas $\rho_{k A 2}$ and $\rho_{k A 4}$ are higher than $\rho_{k}$.

When the power spectrum $\left(\propto\left|v_{k}\right|^{2}\right)$ is correctly regularized by the 0th-order subtraction term $\left|v_{k}^{(0)}\right|^{2}$, the spectral energy density $\left(\propto\left|v_{k}^{\prime}\right|^{2}+k^{2}\left|v_{k}\right|^{2}+m^{2} a^{2}\left|v_{k}\right|^{2}\right) \propto \bar{\omega}+\frac{\left(1-4 \nu^{2}\right)^{2}}{128 \bar{\omega}^{5}}$ will be also correctly regularized by the 0th-order subtraction term $\left|v_{k}^{(0)}\right|^{2}+k^{2}\left|v_{k}^{(0)}\right|^{2}+m^{2} a^{2}\left|v_{k}^{(0)}\right|^{2} \propto \bar{\omega}$.

With regard to renormalization for the conformally-coupling massive scalar field, the divergent terms of $\left\langle T^{\mu}{ }_{\nu}\right\rangle$ in (84) and (86) are of the 0th-order (no time derivatives of $a(\tau)$ ), and will be absorbed by renormalizing the cosmological constant $\Lambda$. Neither the gravitation constant nor the counter terms ${ }^{(1)} H_{\mu \nu},{ }^{(2)} H_{\mu \nu}$ are involved in the renormalization [7].

Now we investigate the conformally-coupling massless scalar field. With $m=0$ and $\xi=\frac{1}{6}$, the exact mode (9) reduces to

$$
v_{k}(\tau) \equiv-i \sqrt{\frac{\pi}{2}} \sqrt{\frac{x}{2 k}} H_{1 / 2}^{(2)}(x)=\frac{1}{\sqrt{2 k}} e^{-i k \tau},
$$

Eqs.(19) (21) reduce to

$$
\rho_{k}=\frac{k^{3}}{4 \pi^{2} a^{4}}\left(\left|v_{k}^{\prime}\right|^{2}+k^{2}\left|v_{k}\right|^{2}\right)=\frac{k^{3}}{4 \pi^{2} a^{4}} k, \quad p_{k}=\frac{1}{3} \rho_{k},
$$

and the trace $\left\langle T_{\mu}^{\mu}\right\rangle_{k}=0$. The spectral energy density and pressure in (110) have only one term, like in the Minkowski spacetime. The 0th-order subtraction terms (C.1) (C.2) for $m=0$ (independent of $\xi$ ) are give by

$$
\rho_{k A 0}=\frac{k^{3}}{4 \pi^{2} a^{4}} k, \quad p_{k A 0}=\frac{k^{3}}{12 \pi^{2} a^{4}} k,
$$

just equal to the unregularized stress tensor (110). Thus, the regularized stress tensor is zero, so is the regularized trace,

$$
\left\langle T^{\mu \nu}\right\rangle_{k r e g}=0, \quad\left\langle T_{\beta}^{\beta}\right\rangle_{k r e g}=0 .
$$

Hence, there is no trace anomaly for the conformally-coupling massless scalar field, and the 0thorder regularization respects the conformal symmetry. The power spectrum has only one term,

$$
\Delta_{k}^{2}=\frac{k^{3}}{2 \pi^{2} a^{2}}\left(\left|v_{k}(\tau)\right|^{2}\right)=\frac{k^{3}}{2 \pi^{2} a^{2}} \frac{1}{2 k},
$$


which is UV divergent, the 0th-order regularization is also sufficient, giving a vanishing regularized spectrum

$$
\Delta_{k r e g}^{2}=\frac{k^{3}}{2 \pi^{2} a^{2}}\left(\left|v_{k}(\tau)\right|^{2}-\left|v_{k}^{(0)}(\tau)\right|^{2}\right)=\frac{k^{3}}{2 \pi^{2} a^{2}}\left(\frac{1}{2 k}-\frac{1}{2 k}\right)=0 .
$$

The vanishing results (112) (114) tell us that the 0th-order adiabatic regularization of the conformallycoupling massless scalar field in de Sitter space is essentially the same as the conventional regularization (by normal ordering) of the massless scalar field in Minkowski spacetime.

It is an interesting observation that, for the case $m=0$ and $\xi=\frac{1}{6}$, the 2nd- and 4th-order subtraction terms (C.4) (C.5) (C.6) (C.7) are actually equal to the 0th-order terms,

$$
\rho_{k A 4}=\rho_{k A 2}=\rho_{k A 0}, \quad p_{k A 4}=p_{k A 2}=p_{k A 0},
$$

and consequently, the 0th-, 2nd-, and 4th-order regularization are the same. This is true not only for de Sitter space, and but also for a general flat RW spacetime, as is seen from the expressions (C.4) (C.5) (C.6) (C.7). The relations (115) can be also rechecked as follows. For $m=0$ and $\xi=\frac{1}{6}$, the 0th-order effective frequency $W^{(0)}=k$, and the 0th-order WKB solution

$$
v_{k}^{(0)}=\frac{1}{\sqrt{2 W^{(0)}}} e^{-i \int W^{(0)} d \tau}=\frac{1}{\sqrt{2 k}} e^{-i k \tau},
$$

which is equal to the exact mode $v_{k}$ in (109). The 2 nd-order by (B.8) is $W^{(2)}=k$, and the 4 th-order $W^{(4)}=k$ by (B.18), so that

$$
v_{k}^{(2)}=v_{k}^{(4)}=v_{k}^{(0)} .
$$

Thus, for the conformally-coupling massless scalar field in a general RW spacetime, the WKB approximate solution (116) is the exact solution, as is evident from the wave equation (5), the regularized spectral stress tensor and power spectrum will be always zero under adiabatic regularization.

The zero trace (112) can also follow from taking the massless limit of 0th-order regularized trace of a massive field. In contrast, the so-called conformal trace anomaly in literature was made up from the massless limit of 4th-order regularized trace of a massive field. The stress tensor (19) (21) (23) of the massive scalar field contains the mass $m$ as a smooth parameter. Starting with $m \neq 0$, the unregularized trace (17) (23) with $\xi=\frac{1}{6}$ give

$$
\left\langle T^{\mu}{ }_{\mu}\right\rangle_{k}=\frac{k^{3}}{2 \pi^{2} a^{2}} m^{2}\left|v_{k}\right|^{2},
$$

the 0th-order subtraction term for the trace in (C.3) is

$$
\left\langle T_{\mu}^{\mu}\right\rangle_{k A 0}=\frac{m^{2} k^{3}}{2 \pi^{2} a^{2}} \frac{1}{2 \omega}
$$

so, the 0th-order regularized trace is

$$
\left\langle T_{\mu}^{\mu}\right\rangle_{k}-\left\langle T_{\mu}^{\mu}\right\rangle_{k A 0}=\frac{m^{2} k^{3}}{2 \pi^{2} a^{2}}\left(\left|v_{k}\right|^{2}-\frac{1}{2 \omega}\right),
$$

which holds for $\xi=\frac{1}{6}$ and a general $m$. Now taking the limit $m=0$ of (120), using $\omega=k$ and $\left|v_{k}\right|^{2}=\frac{1}{2 k}$ by (109), one obtains

$$
\left\langle T_{\mu}^{\mu}\right\rangle_{k}-\left\langle T_{\mu}^{\mu}\right\rangle_{k A 0}=\lim _{m \rightarrow 0} \frac{m^{2} k^{3}}{2 \pi^{2} a^{2}}\left(\frac{1}{2 k}-\frac{1}{2 k}\right)=0,
$$

and its $k$-integration is also zero, $\left\langle T^{\mu}{ }_{\mu}\right\rangle_{\text {reg }}=0$. In Sect.6, we shall rederive the results (112) (114) by the method of regularization of Green's function. The trace anomaly in adiabatic regularization [27-29] occurred in the finite part of 4th-order adiabatic subtraction term (C.8) for $\xi=\frac{1}{6}$. Since the 4th-order regularization leads to a negative spectral energy density, the trace anomaly is a consequence of the improper prescription. 


\section{The regularization of scalar field with a general $\xi$}

For a massive scalar field with a general coupling $\xi$, the situation of adiabatic regularization is more complicated than the $\xi=0$ and $\xi=\frac{1}{6}$ cases in Sects. 3, 4. In this section we consider the value of $\xi$ in the open interval $\left(0, \frac{1}{6}\right)$ in de Sitter space. We briefly report the calculation results.

First we find that the 0th-order regularization fails to remove UV divergences in the spectral energy density and power spectrum.

Next we find that the power spectrum is negative for $\xi$ in $\left(0, \frac{1}{6}\right)$ and for a general $m$, by either the 2nd- or 4th-order regularization, as shown in Figure 6(a), Figure 6(b), Figure 6(c). Comparatively, the 4th-order is more interesting in that it gives a power spectrum which is positive at high $k$, but only has some distortions with negative values at low $k$ around $k|\tau| \lesssim 0.6$.

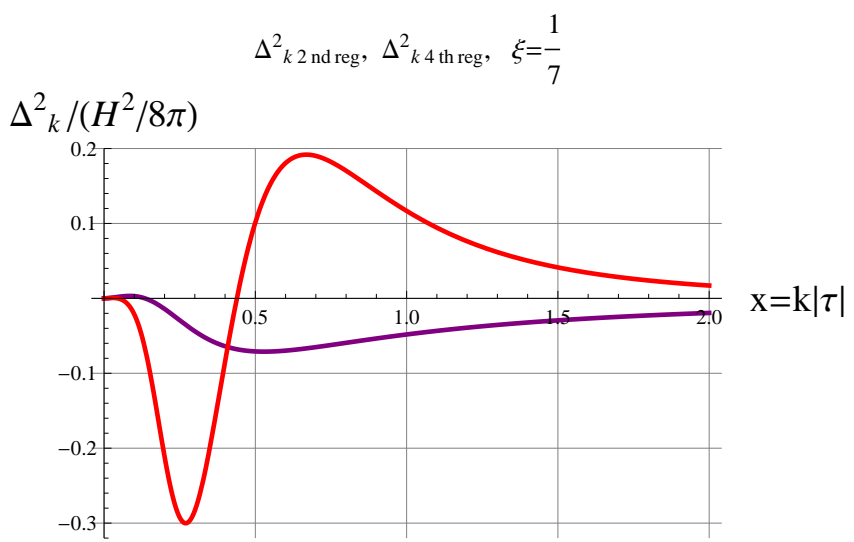

(a)

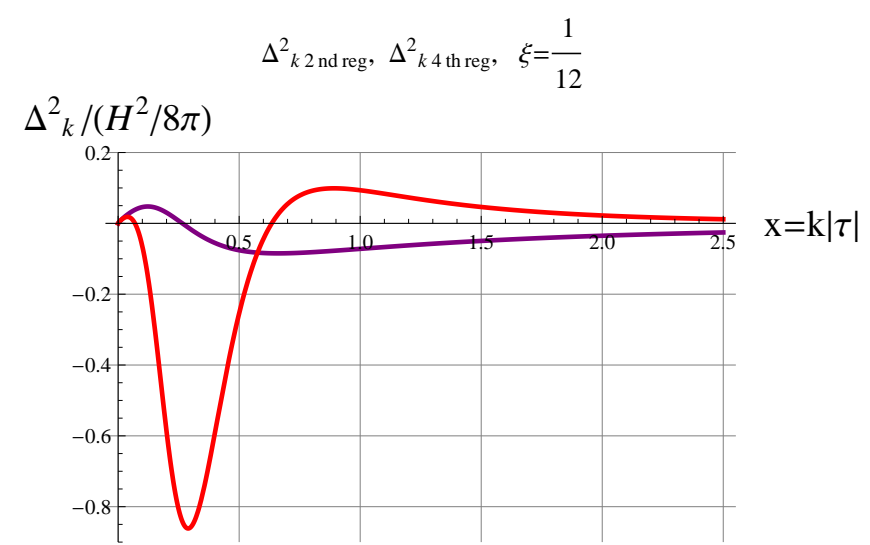

(b)

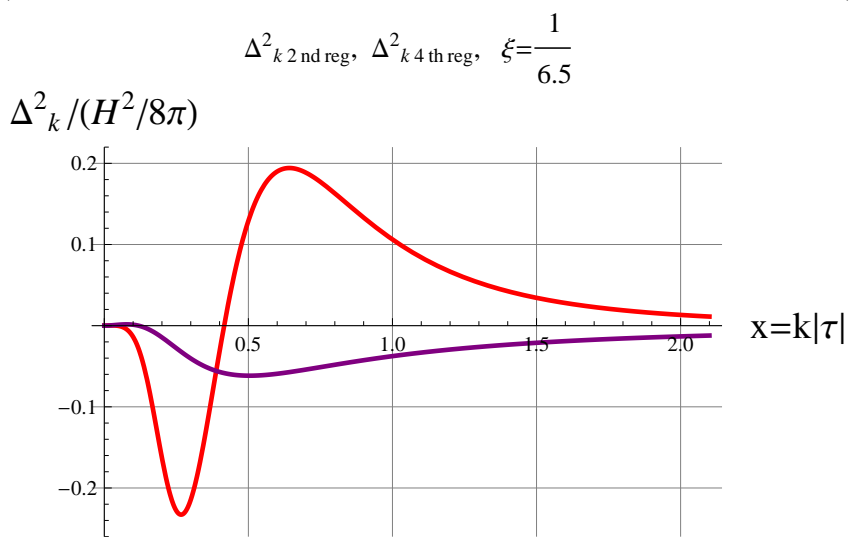

(c)

Figure 6: (a) Red: the 4th-order regularized power spectrum. Purple: the 2nd-order. Both have negative values for $\xi=\frac{1}{7}$. (b) Red: the 4th-order regularized power spectrum. Purple: the 2nd-order. Both have negative values for $\xi=\frac{1}{12}$. (c) Red: the 4th-order regularized power spectrum. Purple: the 2nd-order. Both have negative values for $\xi=\frac{1}{6.5}$.

Then for the spectral energy density, we find that the 2nd-order regularized one is positive for $\xi \in\left(0, \frac{1}{12}\right]$ as shown in Figure 7(a), and is positive at high $k$ and negative at low $k$ for $\xi \in\left(\frac{1}{12}, \frac{1}{6.5}\right)$ as shown in Figure $7(\mathrm{~b})$, and is negative at high $k$ for $\xi \in\left(\frac{1}{6.5}, \frac{1}{6}\right)$, as shown in Figure 7(c) and Figure $7(\mathrm{~d})$. The 4th-order regularization leads to a spectral energy density which is positive at high $k$, and has some distortions with negative values at low $k$ around $k|\tau| \lesssim 0.6$, as shown in Figure 7 (c), Figure 8(a), Figure 8(b).

The above results for $\xi \in\left(0, \frac{1}{6}\right)$ with $m \neq 0$ tells that the conventional adiabatic regularization can not give simultaneously positive spectral energy density and positive power spectrum by the 


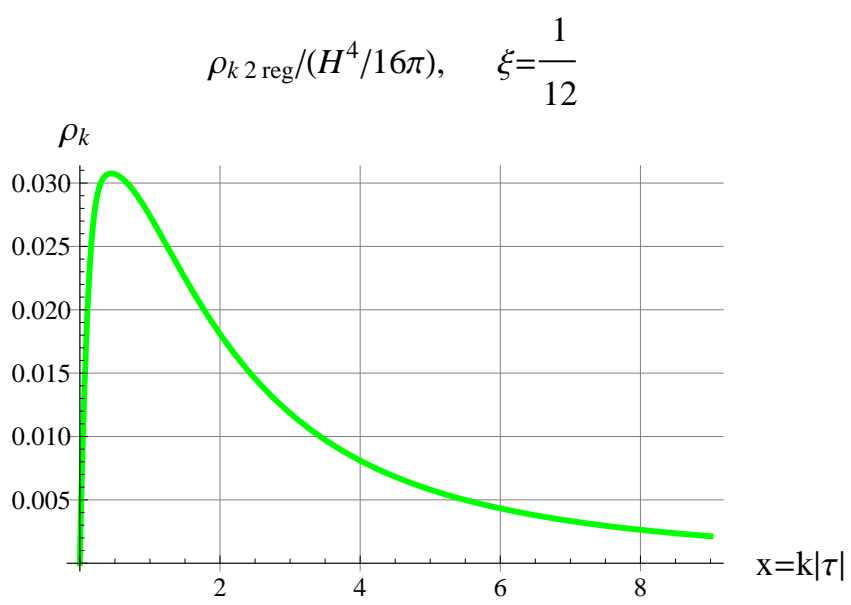

(a)

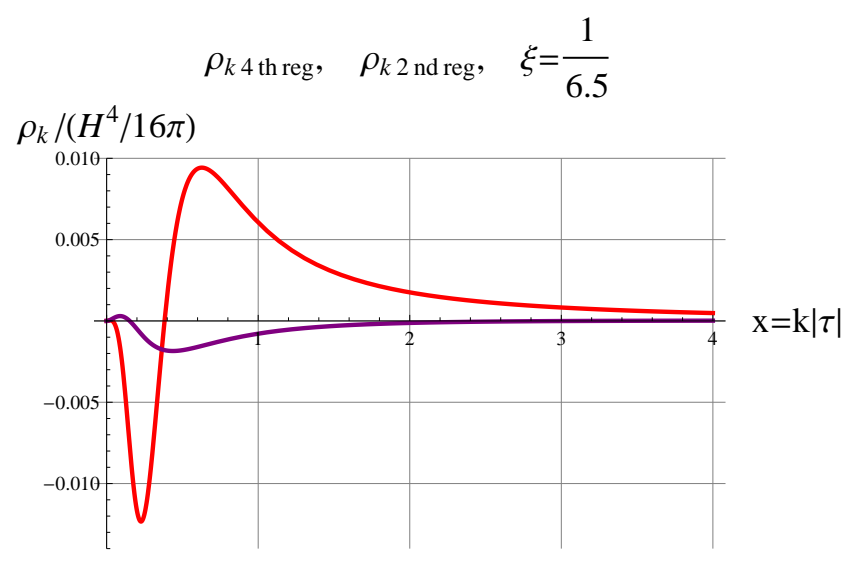

(c)

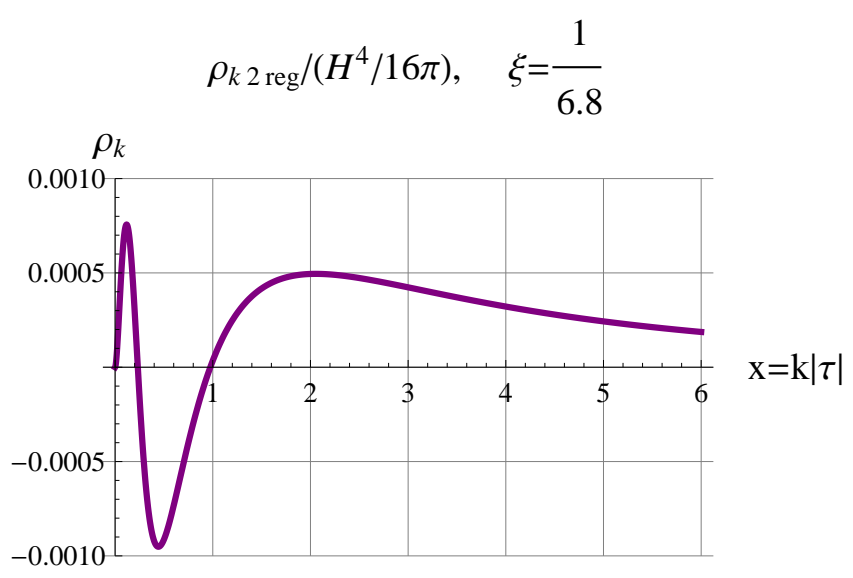

(b)

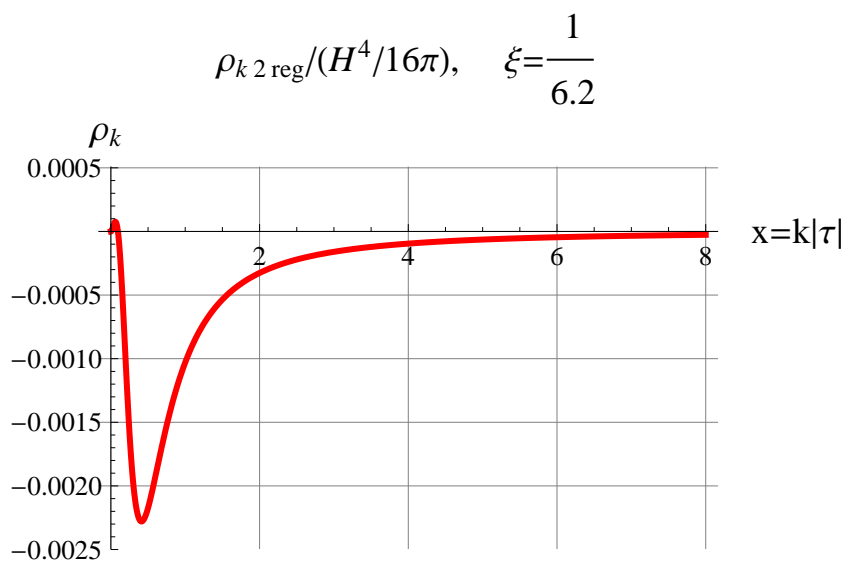

(d)

Figure 7: (a) The 2nd-order regularized $\left(\rho_{k}-\rho_{k A 2}\right)$ is positive definite for $\xi=\frac{1}{12}$. (b) The 2nd-order $\left(\rho_{k}-\rho_{k A 2}\right)$ is positive at high $k$ and negative at low $k$ for $\xi=\frac{1}{6.8}$. (c) The regularized spectral energy densities have negative values for $\xi=\frac{1}{6.5}$. Red: the 4th-order; Purple: the 2nd-order. (d) The 2nd-order $\left(\rho_{k}-\rho_{k A 2}\right)$ is negative at high $k$ for $\xi=\frac{1}{6.2}$.

same adiabatic order. The results also suggest that only the 4th-order regularization is hopeful in that it gives positive spectra at high $k$, while the distortions and negative values occur only at low $k$ outside the horizon of inflation. In order to get positive spectra without low $k$ distortions, some modifications may be made to the 4 th-order regularization for a general $\xi$, like what we have done for RGW [36].

For a massless scalar field with $\xi \in\left(0, \frac{1}{6}\right)$ in de Sitter space, we find that the 0th-order regularization again fails to remove UV divergences. The 2nd-order subtraction terms (C.4) (C.5) for the stress tensor turn out to be equal to the 4th-order (C.6) (C.7),

$$
\begin{gathered}
\rho_{k A 4}=\rho_{k A 2}=\frac{k^{3}}{4 \pi^{2} a^{4}}\left[k+\left(\xi-\frac{1}{6}\right) \frac{-3}{k \tau^{2}}\right], \\
p_{k A 4}=p_{k A 2}=\frac{k^{3}}{12 \pi^{2} a^{4}}\left[k+\left(\xi-\frac{1}{6}\right) \frac{1}{k} \frac{3}{\tau^{2}}\right],
\end{gathered}
$$

and the regularized spectral energy density is positive and UV convergent, as shown in Figure 8(c) for $\xi=\frac{1}{12}$ as an illustration.

However, for the power spectrum, the 2nd- and 4th-order subtraction terms are not equal, $\left|v_{k}^{(2)}\right|^{2} \neq\left|v_{k}^{(4)}\right|^{2}$ (see (B.10) and (B.20)). The 2nd-order regularized power spectrum is negative, 


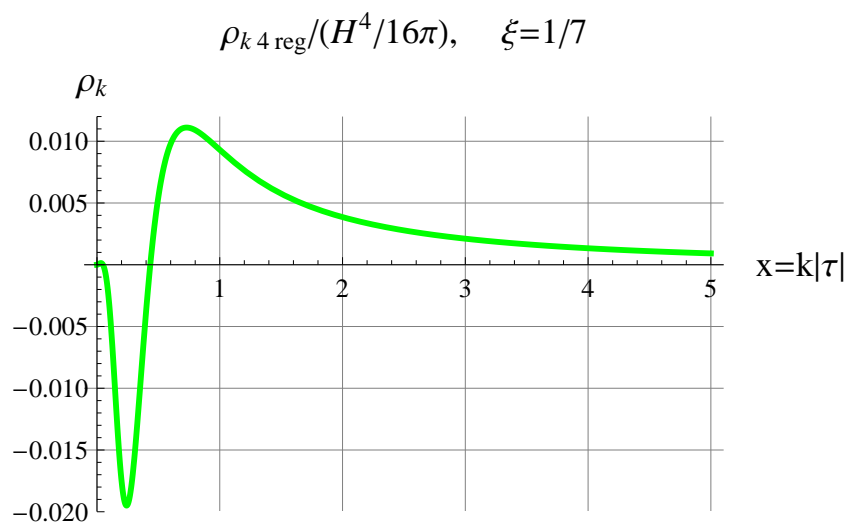

(a)

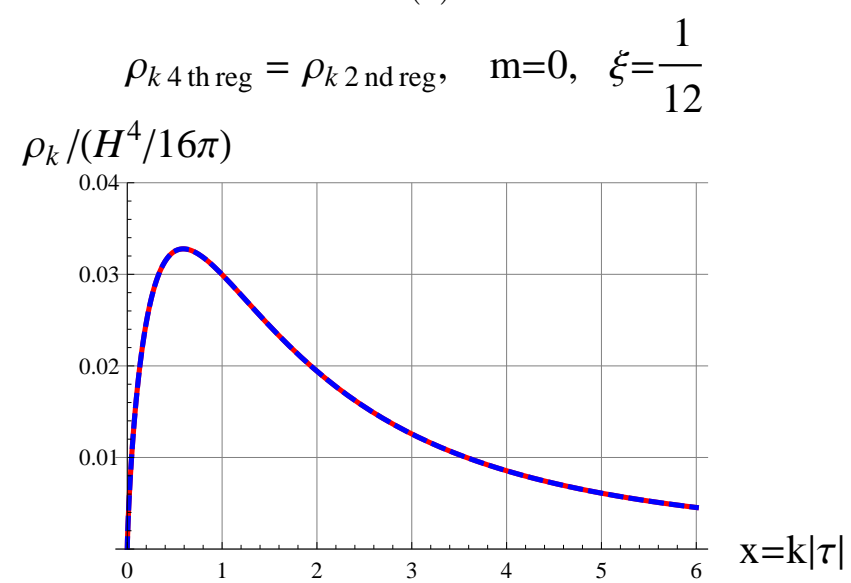

(c)

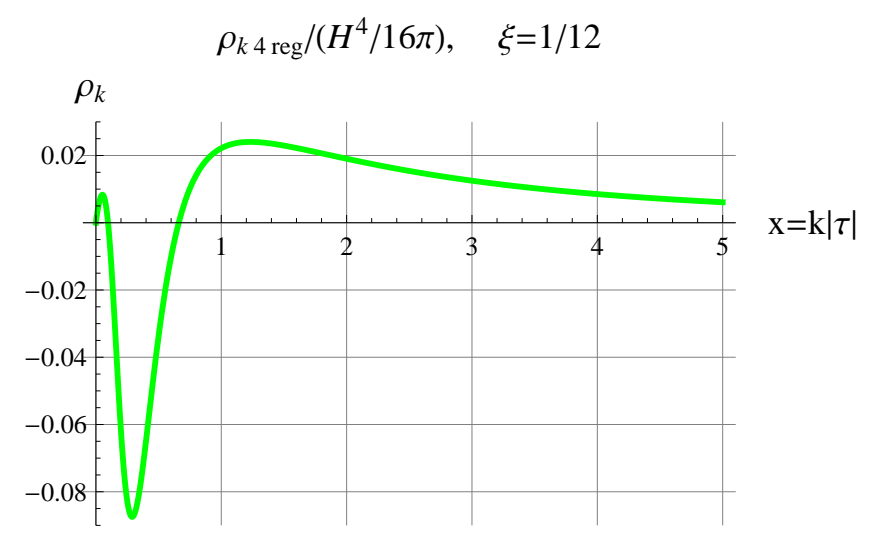

(b)

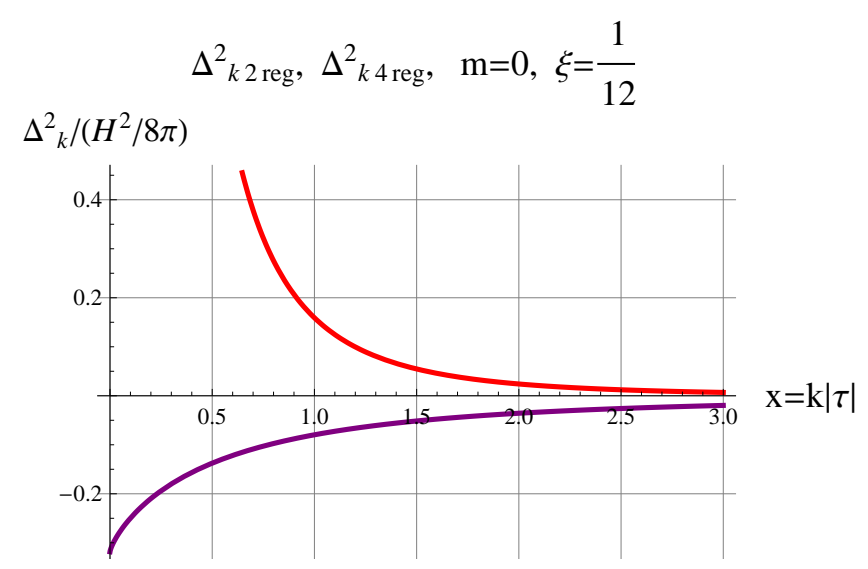

(d)

Figure 8: (a) The 4th-order $\left(\rho_{k}-\rho_{k A 4}\right)$ is positive at high $k$ and negative at low $k$ for $\xi=\frac{1}{7}$. (b) The 4 th-order $\left(\rho_{k}-\rho_{k A 4}\right)$ is positive at high $k$ and negative at low $k$ for $\xi=\frac{1}{12}$. (c) The 2nd- and 4th-order regularized spectral energy densities are overlapped, both positive and UV-convergent for $m=0$ and $\xi=\frac{1}{12}$. (d) Purple: the 2nd-order is negative; Red: the 4th-order regularized power spectrum is positive and UV-convergent, but is IR divergent for $m=0$ and $\xi=\frac{1}{12}$.

and the 4th-order one is positive and UV convergent but suffers from IR divergence, as shown in Figure 8(d). This IR divergence is due to the zero mass, analogous to that of RGW [36]. Some modifications at low $k$ are needed to save the 4th-order regularization. Overall, the issue of adiabatic regularization for a general $\xi \in\left(0, \frac{1}{6}\right)$ is unsettled.

\section{The Green's function regularization with $\xi=0$ and $\frac{1}{6}$}

The 2-point Green's function and the power spectrum are the Fourier transformation of each other, and they contain the same information and are complementary to each other. In de Sitter space with the mode solution (9), the unregularized Green's function in the vacuum for a general $\nu$ is

$$
\begin{aligned}
G\left(x^{\mu}, x^{\prime \mu}\right) & =\frac{1}{(2 \pi)^{3}} \int d^{3} k e^{i \mathbf{k} \cdot\left(\mathbf{r}-\mathbf{r}^{\prime}\right)} \phi_{k}(\tau) \phi_{k^{\prime}}^{*}\left(\tau^{\prime}\right) \\
& =\frac{|\tau|^{1 / 2}\left|\tau^{\prime}\right|^{1 / 2}}{8 \pi a(\tau) a\left(\tau^{\prime}\right)} \frac{1}{\left|r-r^{\prime}\right|} \int_{0}^{\infty} d k k \sin \left(k\left|r-r^{\prime}\right|\right) H_{\nu}^{(1)}(k \tau) H_{\nu}^{(2)}\left(k \tau^{\prime}\right) .
\end{aligned}
$$


The integration (124) can be carried out [14,38], and the result can be written in terms of a hypergeometric function as the following

$$
G(\sigma)=\frac{H^{2}}{16 \pi^{2}} \Gamma\left(\frac{3}{2}-\nu\right) \Gamma\left(\nu+\frac{3}{2}\right){ }_{2} F_{1}\left[\frac{3}{2}+\nu, \frac{3}{2}-\nu, 2,1+\frac{\sigma}{2}\right]
$$

where $\sigma=\left[\left(\tau-\tau^{\prime}\right)^{2}-\left(r-r^{\prime}\right)^{2}\right] /\left(2 \tau \tau^{\prime}\right)$. (We have checked the result (125) by integration, and noticed that there should be a factor 2 in Eq.(3.10) in Ref. [14]. See also Refs. [7,8] for another derivation.) We plot $G(\sigma)$ of a massive field in the solid line in Figure 9(a), which is IR convergent but UV divergent. These asymptotic behaviors can be demonstrated analytically as follows. At large $\sigma$ the series expansion of (125) for a general $\nu$ is

$$
G(\sigma)=\frac{H^{2}}{16 \pi^{2}}\left[\sigma^{\nu-3 / 2}\left(\frac{2^{\frac{3}{2}-\nu} \Gamma\left(\frac{3}{2}-\nu\right) \Gamma(2 \nu)}{\Gamma\left(\nu+\frac{1}{2}\right)}\right)+\sigma^{-\nu-3 / 2}\left(\frac{2^{\nu+\frac{3}{2}} \Gamma\left(\frac{3}{2}+\nu\right) \Gamma(-2 \nu)}{\Gamma\left(\frac{1}{2}-\nu\right)}\right)+\ldots\right]
$$

which is IR convergent for general cases $\nu<3 / 2$. At small $\sigma$ the series expansion is

$$
\begin{aligned}
G(\sigma)= & \frac{H^{2}}{16 \pi^{2}}\left[-\frac{2}{\sigma}+\ln \sigma\left(\frac{1}{4}-\nu^{2}\right)+\left(\frac{1}{4}-\nu^{2}\right)\left(\psi^{(0)}\left(\frac{3}{2}+\nu\right)+\psi^{(0)}\left(\frac{3}{2}-\nu\right)+i \pi+2 \gamma-1-\ln 2\right)\right. \\
& \left.+\frac{\sigma}{4}\left(\frac{1}{4}-\nu^{2}\right)\left(\nu^{2}-\frac{9}{4}\right)\left(\ln \sigma+\psi^{(0)}\left(\frac{5}{2}+\nu\right)+\psi^{(0)}\left(\frac{5}{2}-\nu\right)+i \pi+2 \gamma-\frac{5}{2}-\ln 2\right)+\ldots\right]
\end{aligned}
$$

where $1 / \sigma$ and $\ln \sigma$ are divergent. The UV divergences are to be removed by regularization.

However, the problem is what part of $G(\sigma)$ should be subtracted off. In literature, one is commonly guided by the series expansion $G(\sigma)$ of $(127)$. But this guide may not insure to work out. Let us compare (127) with the series expansion of power spectrum $\Delta_{k}^{2}$ of (35) (79) at high $k$. Generally, a power of $\sigma$ in (127) is not in a one-to-one correspondence to a power of $\omega$ in $\Delta_{k}^{2}$. For instances, the divergent term $1 / \omega$ can generally contribute to the two divergent terms $-\frac{2}{\sigma}$, $\ln \sigma\left(\frac{1}{4}-\nu^{2}\right)$, and many other terms. On the other hand, the constant term in (127) can receive contributions from all the convergent terms $\omega^{-5}, \omega^{-7}$, etc, that is, by terms of all adiabatic orders. The conventional thinking is to subtract $1 / \sigma, \ln \sigma$ and some finite terms, but this simple subtraction will cause some difficulty to the method of regularization of the Green's function as we shall show soon.

Only in two special cases of the massless scalar field, we successfully carry out the direct regularization of Green's function in position space as the following. First, for $m=0$ and $\xi=\frac{1}{6}$, the Green's function (125) reduces to

$$
G(\sigma)=-\frac{H^{2}}{16 \pi^{2}} \frac{2}{\sigma}
$$

We have also double checked this result by direct integration of (124). (128) has only one term, and corresponds to the unregularized power spectrum (113) that also has only one term. This is like the Green's function of a massless scalar field in the Minkowski spacetime. For the equal-time $\tau=\tau^{\prime}$ case (128) reduces to

$$
G(\sigma)=\frac{H^{2}}{4 \pi^{2}} \frac{\tau^{2}}{\left|r-r^{\prime}\right|^{2}}
$$

Since (128) has only one UV divergent term, the regularized Green's function is simply given by

$$
G(\sigma)_{r e g}=G(\sigma)-G(\sigma)_{s u b}=0
$$


where the subtraction term

$$
G(\sigma)_{s u b}=-\frac{H^{2}}{16 \pi^{2}} \frac{2}{\sigma}
$$

And the regularized auto-correlation function is also zero, $G(0)_{\text {reg }}=\lim _{\sigma \rightarrow 0} G(\sigma)_{r e g}=0$, here the coincidence limit $\sigma \rightarrow 0$ is taken after the subtraction. The zero regularized Green's function (130) and the zero regularized power spectrum (114) agree with each other, as they are the Fourier transformation of each other. The vanishing result (130) subsequently yields, by the relation (101), a zero regularized trace,

$$
\left\langle T_{\beta}^{\beta}\right\rangle_{r e g}=m^{2} G(0)_{r e g}=0 \times 0=0,
$$

and a zero regularized stress tenor,

$$
\left\langle T_{\mu \nu}\right\rangle_{\text {reg }}=\frac{1}{4} g_{\mu \nu}\left\langle T_{\beta}^{\beta}\right\rangle_{\text {reg }}=0,
$$

by the maximal symmetry relation (105). The results (132) (133) are consistent with (112) (121) that has been derived in the $k$-space by adiabatic regularization in Sect.4. Hence, for the conformally-coupling massless scalar field, the regularization of Green's function in the position space, the 0th-order adiabatic regularization, and the massless limit of massive field, all these three procedures yield the same result.

Next, for $m=0$ and $\xi=0$, the factor $\Gamma\left(\frac{3}{2}-\nu\right)$ of expression (127) would give a formal infinity, as its mathematical form is not well presented for $\nu=\frac{3}{2}$. We can directly integrate Eq.(124) to obtain the Green's function

$$
\begin{aligned}
G\left(x^{\mu}, x^{\prime \mu}\right) & =\frac{1}{(2 \pi)^{3}} \frac{1}{a(\tau) a\left(\tau^{\prime}\right)} \int \frac{1}{k} d^{3} k e^{i \mathbf{k} \cdot\left(\mathbf{r}-\mathbf{r}^{\prime}\right)-i k\left(\tau-\tau^{\prime}\right)} \frac{1}{2}\left(1+i\left(\frac{1}{\tau^{\prime}}-\frac{1}{\tau}\right) \frac{1}{k}+\frac{1}{k^{2}} \frac{1}{\tau \tau^{\prime}}\right) \\
& =\frac{1}{8 \pi^{2} a(\tau) a\left(\tau^{\prime}\right)\left|\tau \tau^{\prime}\right|}\left(-\frac{1}{\sigma}-\ln \sigma\right)
\end{aligned}
$$

which is also valid for the coupling massless scalar field with $\nu=\frac{3}{2}$ in a general RW spacetime. In de Sitter space, it is

$$
G(\sigma)=\frac{H^{2}}{8 \pi^{2}}\left(-\frac{1}{\sigma}-\ln \sigma\right) .
$$

This Green's function has two terms just corresponding to the power spectrum (75). The first term is the same as (128) of $\xi=\frac{1}{6}$ case, and the second term is the log term. Both terms are UV divergent at $\sigma \rightarrow 0$, and, moreover, the $\log$ term is also IR divergent at $\sigma \rightarrow \infty$, a behavior just anticipated from the power spectrum (75). Therefore, the appropriate regularization is to remove both terms, and the subtraction term is taken to be

$$
G(\sigma)_{s u b}=\frac{H^{2}}{8 \pi^{2}}\left(-\frac{1}{\sigma}-\ln \sigma\right)
$$

so that

$$
G(\sigma)_{r e g}=G(\sigma)-G(\sigma)_{s u b}=0 .
$$

This zero regularized Green's function and the zero regularized power spectrum (76) agree with each other. The minimally-coupling massless field is a special case in which the $\ln \sigma$ term is removed properly. For the massive scalar field, nevertheless, the $\ln \sigma$ term appearing in the series expansion (127) can not be simply subtracted off, for doing this will cause IR divergence, as we demonstrate in the following.

For general $m$ and $\xi$, we simply do not know what divergent terms to be subtracted off the Green's function in position space even when the analytical $G(\sigma)$ is given. In literature, looking at the series expansion (127), the following regularization was often proposed $[7-9,11,14]$

$$
G(\sigma)_{r e g}=G(\sigma)-G(\sigma)_{s u b}
$$


where the subtraction term was taken to be the Hadamard form as the following

$$
\begin{aligned}
G(\sigma)_{s u b}= & \frac{H^{2}}{16 \pi^{2}}\left[-\frac{2}{\sigma}+\ln \sigma\left(\frac{1}{4}-\nu^{2}\right)\right. \\
& \left.+\left(\frac{1}{4}-\nu^{2}\right)\left(\psi^{(0)}\left(\frac{3}{2}-\nu\right)+\psi^{(0)}\left(\frac{3}{2}+\nu\right)+2 \gamma-1-\ln 2\right)\right] .
\end{aligned}
$$

(As we shall see later, this subtraction term is actually not correct, and the correct subtraction terms is (144) for $\xi=0$, and (148) for $\xi=\frac{1}{6}$, respectively.) By appearance, $G(\sigma)_{\text {sub }}$ contains the same UV divergence as the series expansion of $G(\sigma)$ at small $\sigma$, including a $\ln \sigma$ term which is both UV and IR divergent. The last term of (139) is constant and finite (it contains the 4th-order adiabatic terms.) To examine this conventional prescription, we plot $G(\sigma)_{\text {sub }}$ and $G(\sigma)$ together in Figure 9(a) for a comparison. $G(\sigma)$ itself is actually IR convergent at $\sigma \rightarrow \infty$, and does not have the log IR divergence. To show the difficulty, we plot the regularized $G(\sigma)_{\text {reg }}$ in Figure $9(\mathrm{~b})$, which becomes, nevertheless, IR divergent at large $\sigma$ due to the $\ln \sigma$ term in $G(\sigma)_{\text {sub }}$. Unfortunately, this $\log$ IR divergence was neglected and unaddressed in literature $[7-9,11,12,14,17]$. In fact, as long as one takes the subtraction term in a form as Eq.(139), one inevitably faces this $\ln \sigma$ difficulty for general $m$ and $\xi$.

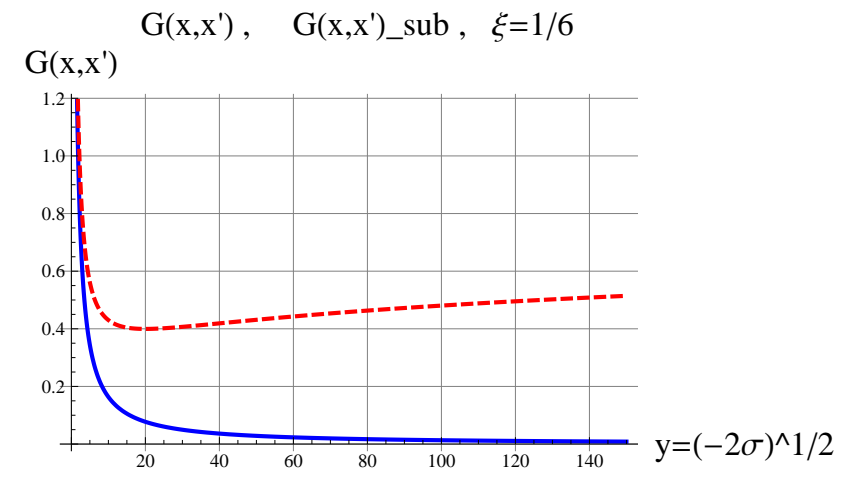

(a)

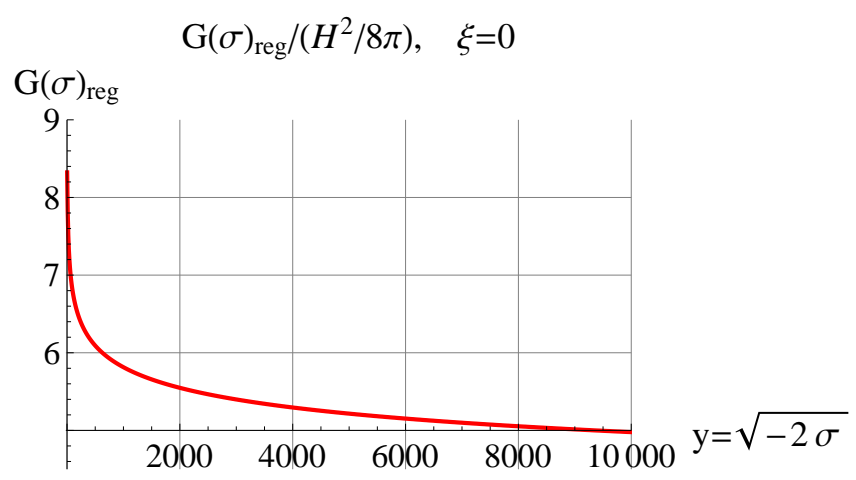

(c)

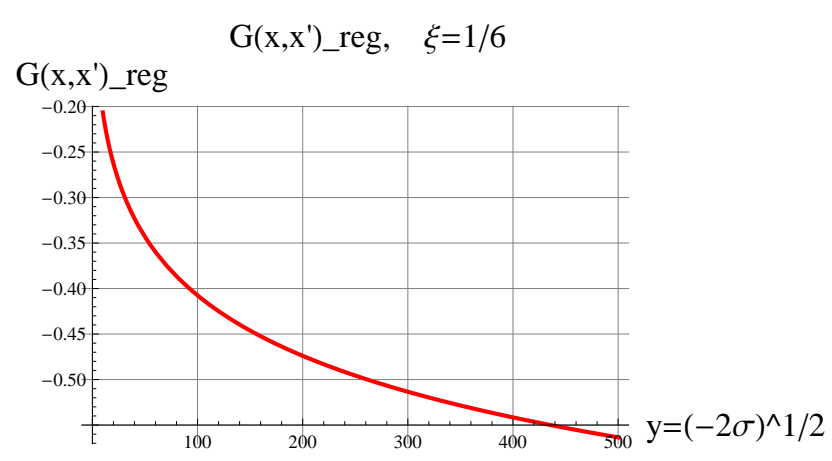

(b)

$\mathrm{G}(\mathrm{y}), \quad \mathrm{G}(\mathrm{y}) \_\mathrm{sub}, \quad \xi=0$

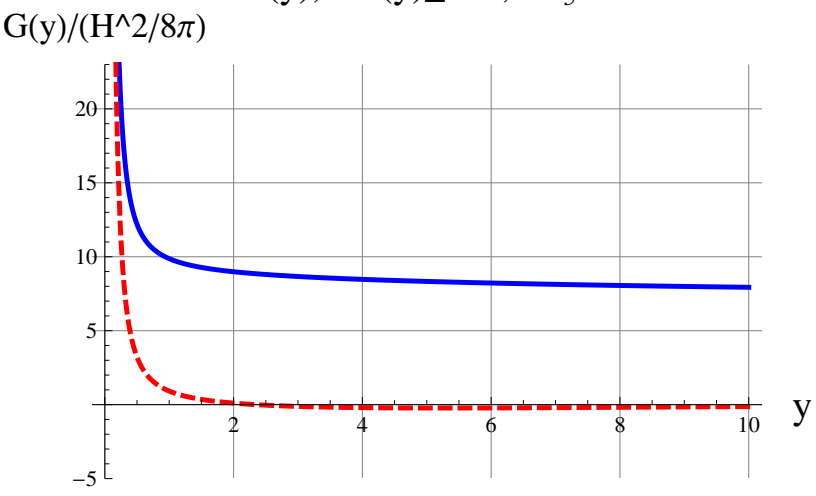

(d)

Figure 9: (a) The model $\xi=\frac{1}{6}$. Blue: the unregularized $G(y)$ in (125). Red Dashed: the subtraction term $G(y)_{s u b}$ in (139). (b) The model $\xi=\frac{1}{6}$. The regularized Green's function prescribed by (138), suffers from the $\log$ IR divergence at $\sigma \rightarrow \infty$ caused by the subtraction term (139). (c) The model $\xi=0$. The 2nd-order regularized $G(y)_{\text {reg }}$ given by (145) is IR- and UV-convergent. (d) The model $\xi=0$. Blue: the unregularized $G(y)$; Red Dashed: the subtraction in Eq.(144).

The difficulty of regularization of the Green's function in position space $[7,8,11,14,17]$ is over- 
come by adiabatic regularization when the latter provides a positive, UV- and IR-convergent power spectrum. For de Sitter space, given a regularized power spectrum, such as (29) (78), we can perform Fourier transformation and convert it into a corresponding regularized Green's function,

$$
G\left(\mathbf{r}, \mathbf{r}^{\prime}\right)_{r e g}=\frac{1}{\left|r-r^{\prime}\right|} \int_{0}^{\infty} \frac{\sin \left(k\left|r-r^{\prime}\right|\right)}{k^{2}} \Delta_{k}^{2}(\tau)_{r e g} d k
$$

where the equal-time case $|\tau|=\left|\tau^{\prime}\right|=1$ is considered for illustration. The definition (140) is analogous to the definition (11).

First, for $\xi=0$, we substitute the 2nd-order regularized $\Delta_{k \text { reg }}^{2}$ of (29) into (140) and have the following

$$
\begin{aligned}
G(y)_{\text {reg }} & =\frac{H^{2}}{8 \pi} \frac{1}{y} \int_{0}^{\infty} d k k \sin (k y)\left(\left|H_{\nu}^{(1)}(k)\right|^{2}-\frac{4}{\pi} \frac{1}{2 W^{(2)}}\right) \\
& =G(y)-G(y)_{\text {sub }}
\end{aligned}
$$

where $G(y)$ with $y \equiv\left|r-r^{\prime}\right|$ is given by (125), and $G(y)_{\text {sub }}$ is the subtraction term and can be calculated as the following. By (36), it involves the following integrations

$$
\int_{0}^{\infty} d k k \sin (k y) \frac{1}{W^{(2)}}=\int_{0}^{\infty} d k k \sin (k y)\left[\frac{1}{\bar{\omega}}+\frac{1}{\bar{\omega}^{3}}+\frac{3 \frac{m^{2}}{H^{2}}}{4 \bar{\omega}^{5}}-\frac{5 \frac{m^{4}}{H^{4}}}{8 \bar{\omega}^{7}}\right] .
$$

By the following integration formulae

$$
\int_{0}^{\infty} d k \frac{k \sin (k y)}{\left(k^{2}+\frac{m^{2}}{H^{2}}\right)^{n+\frac{1}{2}}}=\frac{-\sqrt{\pi}}{2^{n}\left(\frac{m^{2}}{H^{2}}\right)^{n / 2} \Gamma\left(n+\frac{1}{2}\right)} \frac{d}{d y}\left(y^{n} K_{n}\left(y \frac{m}{H}\right)\right)
$$

where $K_{n}$ is the modified Bessel's function (see 3.773 in Ref. [39]), and by the relations: $\frac{d}{d x} K_{0}(x)=$ $-K_{1}(x), \frac{1}{x} \frac{d}{d x}\left(x K_{1}(x)\right)=-K_{0}(x), \frac{1}{x} \frac{d}{d x}\left(x^{2} K_{2}(x)\right)=-x K_{1}(x), \frac{1}{x} \frac{d}{d x}\left(x^{3} K_{3}(x)\right)=-x^{2} K_{2}(x)$ [40], the subtraction term for the Green's function for $\xi=0$ is given by

$$
G(y)_{s u b}=\frac{H^{2}}{4 \pi^{2}}\left[\frac{m}{H} \frac{1}{y} K_{1}\left(\frac{m}{H} y\right)+K_{0}\left(\frac{m}{H} y\right)+\frac{1}{4} \frac{m}{H} y K_{1}\left(\frac{m}{H} y\right)-\frac{1}{24} \frac{m^{2}}{H^{2}} y^{2} K_{2}\left(\frac{m}{H} y\right)\right]
$$

Thus, we obtain the 2nd-order regularized analytical Green's function for $\xi=0$ as the following

$$
\begin{aligned}
G(y)_{\text {reg }}= & \frac{H^{2}}{16 \pi^{2}} \Gamma\left(\frac{3}{2}-\nu\right) \Gamma\left(\nu+\frac{3}{2}\right)_{2} F_{1}\left[\frac{3}{2}+\nu, \frac{3}{2}-\nu, 2,1-\frac{y^{2}}{4}\right] \\
& -\frac{H^{2}}{4 \pi^{2}}\left[\frac{m}{H} \frac{1}{y} K_{1}\left(\frac{m}{H} y\right)+K_{0}\left(\frac{m}{H} y\right)+\frac{1}{4} \frac{m}{H} y K_{1}\left(\frac{m}{H} y\right)-\frac{1}{24} \frac{m^{2}}{H^{2}} y^{2} K_{2}\left(\frac{m}{H} y\right)\right] .
\end{aligned}
$$

We plot the resulting $G(y)_{\text {reg }}$ in Figure 9(c), which exhibits more clearly the UV finiteness and the IR convergence. The finite value at $y=0$ is

$$
G(0)_{r e g} \simeq 8.369 \frac{H^{2}}{8 \pi}
$$

for the model $\frac{m^{2}}{H^{2}}=0.1$ and $\xi=0$. For a better understanding of this subtraction, in Figure $9(\mathrm{~d})$ we plot the subtraction $G(y)_{\text {sub }}$ given by (144) together with the unregularized $G(y)$. It is seen that $G(y)_{\text {sub }}$ approaches to $G(y)$ from below at small $y$, and thus removes the UV divergences of $G(y)$. At large $y, G(y)_{\text {sub }}$ is lower and approaches to zero faster than $G(y)$, and therefore does not 
affect the IR convergence of $G(y)$. This is a desired pattern, in sharp contrast to the conventionally prescribed subtraction (139) shown in Figure 9(a) by the Green's function in position space.

Next, for $\xi=\frac{1}{6}$, we substitute the 0th-order regularized $\Delta_{k \text { reg }}^{2}$ of (78) into (140) and have the following

$$
\begin{aligned}
G(y)_{r e g} & =\frac{H^{2}}{8 \pi} \frac{1}{y} \int_{0}^{\infty} d k k \sin (k y)\left(\left|H_{\nu}^{(1)}(k)\right|^{2}-\frac{4}{\pi} \frac{1}{2 \omega}\right) \\
& =G(y)-G(y)_{s u b}
\end{aligned}
$$

where $G(y)$ is given by (125) and the subtraction term is

$$
G(y)_{s u b}=\frac{H^{2}}{4 \pi^{2}} \frac{m}{H} \frac{1}{y} K_{1}\left(\frac{m}{H} y\right) .
$$

(Ref. [25] adopted the 4th-order regularization, used an approximate subtraction term valid only at small $y$, and derived the asymptotic expression of $G(y)_{s u b}$ to the order of $y^{2}$.) The 0th-order regularized analytical Green's function for $\xi=\frac{1}{6}$ as the following

$$
\begin{aligned}
G(y)_{r e g}= & \frac{H^{2}}{16 \pi^{2}} \Gamma\left(\frac{3}{2}-\nu\right) \Gamma\left(\nu+\frac{3}{2}\right)_{2} F_{1}\left[\frac{3}{2}+\nu, \frac{3}{2}-\nu, 2,1-\frac{y^{2}}{4}\right] \\
& -\frac{H^{2}}{4 \pi^{2}} \frac{m}{H} \frac{1}{y} K_{1}\left(\frac{m}{H} y\right) .
\end{aligned}
$$

The result is plotted in Figure 10(a), which shows the UV finiteness and IR convergence. The finite

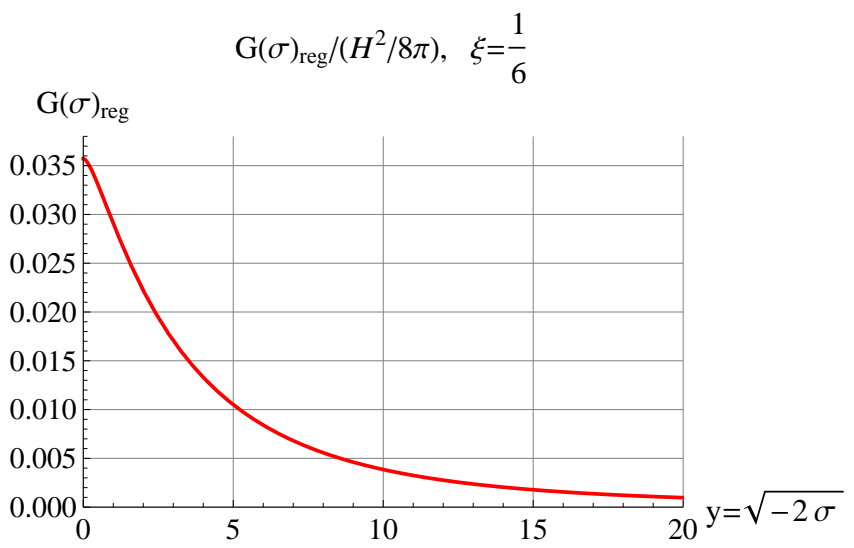

(a)

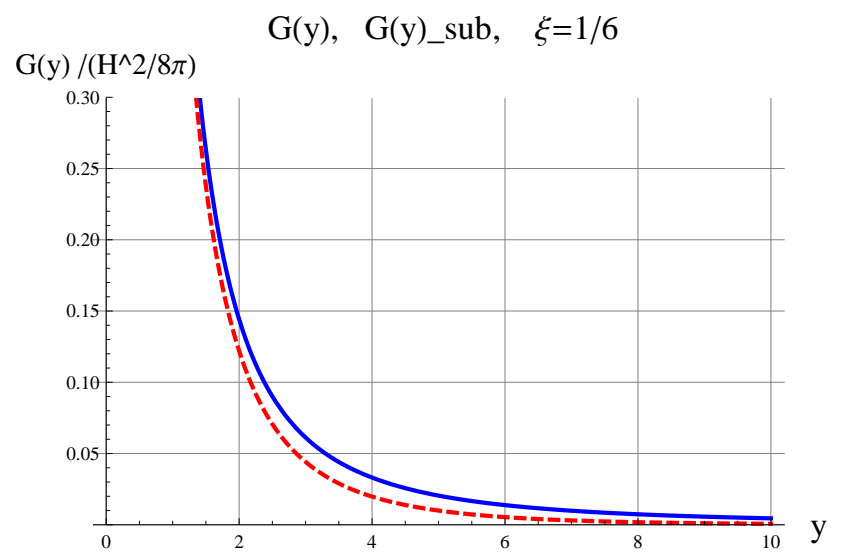

(b)

Figure 10: The model $\xi=\frac{1}{6}$. (a) The 0th-order regularized $G(y)_{\text {reg }}$ in Eq.(149) is IR- and UV-convergent. (b) Blue: the unregularized $G(y)$. Red Dashed: the subtraction term $G(y)_{\text {sub }}$ in Eq.(148).

value at $y=0$ is

$$
G(0)_{r e g}=0.03572 \frac{H^{2}}{8 \pi}
$$

for the model $\frac{m^{2}}{H^{2}}=0.1$ and $\xi=\frac{1}{6}$. This, together with the relation $\left\langle T_{\mu}^{\mu}\right\rangle_{\text {reg }}=m^{2} G(0)_{\text {reg }}$ of $(101)$, gives a value of regularized trace $\left\langle T^{\mu}{ }_{\mu}\right\rangle_{r e g}$ which agrees with that given by (104). In Figure 10(b) we also plot the subtraction $G(y)_{\text {sub }}$ given by (148) and the unregularized $G(y)$. When $m=0,(101)$ yields a zero trace $\left\langle T_{\beta}^{\beta}\right\rangle_{r e g}=0$, which again agrees with (112) (121) derived from the 0th-order adiabatic regularization method in Sect 4.

Hence, for the minimally- and conformally-coupling massive scalar fields in de Sitter space, via the Fourier transformation of regularized power spectra, we have achieved the regularized, analytical 
Green's functions in position space, which are UV as well as IR convergent. We expect that, in general, when a regularized power spectrum is available, the Fourier transformation will provide a viable procedure to obtain the corresponding regularized Green's function.

The subtraction terms (144) (148) are formed from the modified Bessel's functions which are a special combination of an infinite number of powers of $y$ when expanded at small $y$. All these terms as a whole are necessary to ensure the UV convergence and as well as the IR convergence, also to ensure the covariance of regularized Green's function, and the covariant conservation of regularized stress tensor. This inspection explains why the conventional prescription based on the Green's function in position space will not work in general, because one does not know what the proper substraction term should be exactly. The conventional prescription is guided by the series expansion at small $y$, and proposes to subtract only the several divergent terms in the series, such as $1 / y$ and $\ln y$. And that does not work out.

In the remaining of this section we examine the issue of trace anomaly that has been claimed in literature. The trace anomaly of the scalar field with $\xi=\frac{1}{6}$ was first mentioned in Ref. [8] using the Green's function for a massive scalar field in the limit $m=0$. In the following we go into details and investigate its occurrence in the regularization of the Green's function $[8-15,17,25,28]$. In particular, we shall see how it was brought about by an invalid procedure of taking massless limit of the 4th-order adiabatic subtraction term.

To get the trace anomaly in de Sitter space, Ref. [14] started with the incorrect subtraction term (139), and expanded its the constant part at large $m^{2} / H^{2}$,

$$
\left(\frac{1}{4}-\nu^{2}\right)\left(\psi^{(0)}\left(\frac{3}{2}-\nu\right)+\psi^{(0)}\left(\frac{3}{2}+\nu\right)\right) \simeq-\frac{H^{2}}{15 m^{2}}-\frac{8 H^{4}}{315 m^{4}}-\frac{m^{2} \ln \frac{H^{2}}{m^{2}}}{H^{2}}+\frac{2}{3}+\ldots
$$

plugged (139) into (138) to cancel the UV divergent $1 / \sigma$ and $\ln \sigma$ terms,

$$
\begin{aligned}
G(\sigma)_{r e g}= & \frac{H^{2}}{16 \pi^{2}} \Gamma\left(\frac{3}{2}-\nu\right) \Gamma\left(\nu+\frac{3}{2}\right)_{2} F_{1}\left[\frac{3}{2}+\nu, \frac{3}{2}-\nu, 2,1+\frac{\sigma}{2}\right] \\
& -\frac{H^{2}}{16 \pi^{2}}\left[-\frac{2}{\sigma}+\ln \sigma\left(\frac{1}{4}-\nu^{2}\right)-\frac{H^{2}}{15 m^{2}}+\ldots\right]
\end{aligned}
$$

and, after taking the coincidence limit $\sigma \rightarrow 0$, got the regularized stress tensor, keeping up to the order of $H^{4}$ (the adiabatic 4th-order),

$$
\left\langle T_{\mu}^{\mu}\right\rangle_{\text {reg }}=m^{2} G(\sigma=0)_{\text {reg }}=m^{2} \frac{H^{2}}{16 \pi^{2}}\left[\frac{H^{2}}{15 m^{2}}+\ldots\right]
$$

then took the limit $m=0$ and claimed that the conformally-coupling massless scalar field has the following nonzero trace (i.e., the trace anomaly) [14]

$$
\lim _{m=0}\left\langle T_{\mu}^{\mu}\right\rangle_{r e g}=\frac{H^{4}}{240 \pi^{2}}=\frac{R^{2}}{34560 \pi^{2}} .
$$

Thus the appearance of trace anomaly is caused by two procedures: starting with a presumed massive field for a massless field, and deploying the 4th-order subtraction term. These have been commonly adopted in literature which claimed the trace anomaly. Inspection of the above procedures reveals several problems. First, if one works directly with a massless scalar field with $\xi=\frac{1}{6}$, instead of starting with an artificially presumed massive field, one simply obtains a vanishing $G(\sigma)_{\text {reg }}=0$, and will never run into a trace anomaly, as demonstrated by the paragraph of (128) through (133). Second, the subtraction term (139) is incorrect for a massive field, as it contains the $\ln \sigma$ term and will generate the ln IR divergence, as we have pointed earlier. Third, even if the subtraction term (139) were allowed, it will generally lead to negative power spectrum and negative spectral energy 
density, which are not acceptable for physics. Consequently, for general $\xi$, regularization of Green's function in position space has not been accomplished yet, in the sense that the methods have not been able to yield an explicit expression of UV and IR convergent Green's function $G(\sigma)$ even in de Sitter space. In fact, the adequate subtraction terms of the Green's function are given by (144) for $\xi=0$, and respectively by (148) for $\xi=\frac{1}{6}$, which have been derived by Fourier transformation of the adiabatic subtraction terms for power spectra from adiabatic regularization.

For a general curved spacetime, the analytical solution of Green's function may be not available. Often in literature, the Green's function is written formally as the DeWitt-Schwinger proper-time integration $[19,20]$

$$
G(\sigma) \propto \sum_{n=0}^{\infty} a_{n}\left(-\frac{\partial}{\partial m^{2}}\right)^{n} \int_{0}^{\infty} \frac{d s}{s^{2}} \exp \left[-i\left(m^{2} s-\frac{\sigma}{2 s}\right)\right]
$$

As already pointed in Refs. $[11,12,16,20]$, the $s$-integration $(155)$ is undefined at $m=0$ and is valid only at $m \neq 0$. So, the series expansion of $G(\sigma)$ at $m=0$ is not known in this formulation. This is the limitation of DeWitt-Schwinger proper-time integration, and that is why in literature strangely a massive field was started with to get the trace anomaly of a massless field. (By the way, we like to say that the DeWitt-Schwinger integration is not necessary to study the Green's function, and that one can study the Green's function of a massless field in terms of a Hadamard series.) At $m \neq 0,(155)$ can be expanded as a series in the following form $[8,11,20]$

$$
\begin{aligned}
G(\sigma) \propto & \frac{1}{4 \pi^{2}}\left\{a_{0}\left[\frac{1}{\sigma}+m^{2}\left(\gamma+\frac{1}{2} \ln \left|\frac{1}{2} m^{2} \sigma\right|\right)\left(1+\frac{1}{4} m^{2} \sigma+\ldots\right)-\frac{1}{2} m^{2} \ldots\right]\right. \\
& -a_{1}\left[\left(\gamma+\frac{1}{2} \ln \left|\frac{1}{2} m^{2} \sigma\right|\right)\left(1+\frac{1}{2} m^{2} \sigma+\ldots\right)-\frac{1}{2} m^{2} \sigma \ldots\right] \\
& +a_{2} \sigma\left[\left(\gamma+\frac{1}{2} \ln \left|\frac{1}{2} m^{2} \sigma\right|\right)\left(1+\frac{1}{8} m^{2} \sigma+\ldots\right)-\frac{1}{4}-\ldots\right] \\
& \left.+\frac{1}{2 m^{2}}\left[a_{2}+O(\sigma) \ldots\right]+\frac{1}{2 m^{4}}\left[a_{3}+O(\sigma) \ldots\right]+O\left(m^{-6}\right)\right\}
\end{aligned}
$$

which has a structure similar to (127) in de Sitter space. (We must emphasize that this expression is invalid at $m=0$.) Then it was commonly proposed to subtract off some UV divergent terms of (156), such as $a_{0} / \sigma, a_{0} \frac{1}{2} m^{2} \ln \sigma, a_{2} / 2 m^{2}$, etc. (These subtraction terms have been commonly adopted in the methods of regularization in position space, such as the dimensional regularization $[8,9]$, the point-splitting $[11,12,14]$, the zeta function regularization $[8,17]$, and as well as the path-integral formulation [18]. The zeta function regularization in Refs. [8,17] and its path-integral formulation in Ref. [18] are equivalent, and use the same subtraction terms which are explicitly mentioned in Refs. $[8,17]$.) Nevertheless, doing this will lead to an IR divergence since the term $a_{0} \frac{1}{2} m^{2} \ln \sigma$ is also IR divergent, just like in de Sitter space. This is a vital difficulty of regularization guided by the series expansion (156) in position space, and has not been addressed in literature. Without solving this difficulty, one still continued to pick up the subtraction term $a_{2} / 2 m^{2}$ (4th adiabatic order), multiplied by $m^{2}$, took the limit $m=0$, and claimed that the trace anomaly is $\left\langle T^{\mu}{ }_{\mu}\right\rangle=\frac{1}{4 \pi^{2}} a_{2}[8,9,11,14,17]$. But again, this limit-taking procedure is invalid simply because the series (156) does not hold at $m=0$. We emphasize that it is mathematically incorrect to pick up a term in (156) and regard it as a term of $G(\sigma)$ at $m=0$. For instance, the term $\frac{1}{2 m^{4}} a_{3}$ and the subsequent infinitely many terms $\left(\propto m^{-6}, m^{-8}\right.$, etc) will be divergent at $m=0$. The series expansion (156) does not tell what $G(\sigma)$ looks like at $m=0$, nor if the $a_{2}$ term ever exists in $G(\sigma)$ at $m=0$. Thus, it is invalid to claim that $a_{2}$ is the trace anomaly at $m=0$. The real issue is to find adequate subtraction terms to the Green's function, and this has not been solved yet for general curved spacetimes. 


\section{Conclusion and discussions}

We have studied, in great details, adiabatic regularization of a coupling massive scalar field in de Sitter inflation, and found that the conventional 4th-order regularization subtracts off more than necessary and leads to a negative spectral energy density in a broad range of $k$. A negative spectral energy density is unacceptable in the context of cosmology. For a massive scalar field, the adiabatic expansion is made in powers of frequency $\omega$, whereas the degree of UV divergences is counted in powers of $k$. When the powers of $\omega$ is further expanded in terms of $k$, there are infinitely many powers $k^{-n}$. This is the origin of unmatch of the adiabatic order and the degree of UV divergences for a massive scalar field. So we seek an appropriate prescription which will give a positive (nonnegative), IR- and UV-convergent spectral energy density. As it turns out, the order of regularization depends sensitively on the coupling $\xi$.

For the massive field with $\xi=0$ in Section 3, we find that the 0th- and 4th-order prescriptions fail, and the 2nd-order regularization is sufficient to remove quartic, quadratic, and logarithmic UV divergences of the stress tensor, and simultaneously the quadratic, and logarithmic UV divergences of the power spectrum. The resulting regularized spectral energy density and power spectrum are positive, IR- and UV-convergent. Although nonuniformly distributed in the $k$-modes, the regularized stress tensor in the vacuum is maximally symmetric, and respects the covariant conservation. Thus, in connection to inflation cosmology, the massive scalar field can play a double role: its regularized $\left\langle T_{\mu \nu}\right\rangle_{r e g}$ drives the inflation, while its $k$-modes $\phi_{k}$ are the primordial fluctuations in the vacuum. With regard to renormalization, the 2 nd-order regularization involves renormalization of the cosmological and gravitational constants, and does not need the fourth order time-derivative of metric such as ${ }^{(1)} H_{\mu \nu},{ }^{(2)} H_{\mu \nu}$. In the special case of $m=0=\xi$, the 2 nd-order regularization is the same as the 4th-order one, and gives a zero stress tensor and a zero power spectrum.

For the massive field with $\xi=\frac{1}{6}$ in Section 4 , we find that the 2 nd- and 4 th-order prescriptions fail, and the 0th-order regularization is sufficient to remove UV divergences in the stress tensor and the power spectrum, and the regularized spectra are positive, and IR- and UV-convergent. Like the $\xi=0$ case, the regularized stress tensor is maximally symmetric, but nonuniformly distributed in the $k$-modes. The 0th-order regularization involves only renormalization of the cosmological constant. In the special case $\xi=\frac{1}{6}$ and $m=0$, we find that the prescriptions of 0th-, 2nd-, and 4th-order regularization are the same, all lead to a zero regularized stress tensor and a zero regularized power spectrum, and no trace anomaly exists. Even if we start with $m \neq 0$ and $\xi=\frac{1}{6}$, the regularized trace is $\propto m^{2}$, so that the trace is still zero in the limit $m \rightarrow 0$. Our finding is in contrast to the literature-reported trace anomaly which appeared in the 4th-order regularization of a massive scalar field with $\xi=\frac{1}{6}$ in the limit $m \rightarrow 0$. Since the 4th-order regularization leads to a negative spectral energy density, it is an improper prescription, and the trace anomaly is an artifact.

For the massive field with a general $\xi \in\left(0, \frac{1}{6}\right)$ in Section 5 , the regularized spectra generally have irregular distortions and negative values. It seems no regularization of equal order could achieve positive spectral energy density and power spectrum simultaneously. We also notice that, by the 4th-order regularization, both the spectral energy density and power spectrum are positive at high $k$, whereas distortions with negative values occur only at the low $k$ range outside the horizon. This pattern indicates that one may modify regularization at low $k$ in order to achieve positive spectra, like what we have done for RGW [36]. Regularization for a general $\xi$ remains unsettled and more endeavors are needed.

As a complement to the adiabatic regularization, we have studied the regularization of Green's function in position space in Section 6. DeWitt [20] suggested that "after the infinities have been split off," the effective Lagrangian (expressed in terms of the Green's function) "is both coordinate invariant and conformally invariant," however, he did not tell how to achieve this by an explicit demonstration. In curved spacetimes, the analytical Green's function is generally not available, it 
is hard to implement a direct regularization. In de Sitter space, $G(\sigma)$ for general $m$ and $\xi$ is known as a hypergeometric function, but its direct regularization is still not easy, because one does not know its proper subtraction term. Only in the massless cases $m=0$ with $\xi=\frac{1}{6}$ or $\xi=0, G(\sigma)$ contains one (or two) term which is divergent, we are able to directly regularize it to zero, and no trace anomaly exists. This outcome agrees with the result of zero spectra from the 0th- (or 2nd-) order adiabatic regularization. In the cases $m \neq 0$ with $\xi=\frac{1}{6}$ or $\xi=0$ when the regularized power spectra are available, we perform Fourier transformation, and obtain the regularized Green's function which is UV-finite and IR-convergent. And, as it turns out, the subtraction terms $G(\sigma)_{s u b}$ consist of the modified Hankel functions $K_{n}(\sigma)$ as in Eqs.(144) and (148), not just the commonlysuggested Hadamard form as in Eq.(139) which contains the $\ln \sigma$ term. We demonstrate explicitly how this log IR divergence plagues the Green's function regularization, which is an issue not been addressed in literature. When an adequately regularized power spectrum is available, the Fourier transformation provides a viable way to obtain the regularized Green's function. We also analyze, for de Sitter space, how the literature-reported trace anomaly showed up in the Green's function method, and actually it was generated by the improper 4th-order subtraction term. It should be remarked that, due to the homogeneity and isotropy in de Sitter space, the fluctuations represented by the scalar field is a stationary process in terminology of statistics, so that the Green's function is a function of the separation of two spacetime points, $G\left(x^{\mu}, x^{\mu \prime}\right)=G\left(x^{\mu}-x^{\mu \prime}\right)=G(\sigma)$, as explicitly seen in Eq.(125). In this case, when a locally determined part $G^{L}(\sigma)$ is constructed to satisfy the wave equation in $x$ and in $x^{\prime}[16]$, so does the boundary-condition-dependent part $G^{B}\left(x^{\mu}, x^{\mu \prime}\right) \equiv$ $G(\sigma)-G^{L}(\sigma)$. So the presumption that $G^{B}\left(x^{\mu}, x^{\mu \prime}\right)$ not satisfy the wave equation in $x^{\prime}[15]$ does not happen in de Sitter space. In general curved spacetimes, the analytical solution of $G(\sigma)$ is not known, nor is the appropriate subtraction term, it is premature to claim the trace anomaly. In retrospect, the common thing that led to the trace anomaly in those different approaches consisted of two procedures: starting with an artificially-presumed massive field for a massless field, and adopting the incorrect 4th-order subtraction term. If one directly starts with a massless scalar field, instead of a massive field, one will never run into a trace anomaly, as we have shown in the paragraph of (109) through (114) by adiabatic regularization method, and in the paragraph of (128) through (133) by the Green's function method. Even starting with a massive field with $\xi=\frac{1}{6}$, if one adopts the 0th-order regularization, instead of the 4th-order, one will never run into the trace anomaly nor the negative spectral energy density.

Our study shows the great advantage of adiabatic regularization which works particularly in RW spacetimes. When the exact solution of field in a RW spacetime is given, all UV-divergent terms are isolated as powers of $k$, while the subtraction terms of various orders are always given explicitly by WKB approximation. With these in hand, one is able to see if the spectra are UVand IR-convergent and if a spectrum is negative at certain order. Therefore, one is more effectively guided in choosing a proper regularization scheme. Furthermore, the spectra of a massive field are functions of $\omega$, adiabatic subtraction can be implemented without causing the log IR divergence. These important features of adiabatic regularization are not shared by the direct regularization of Green's function in position space. Finally we remark that the regularized stress tensor and Green's function obtained by adiabatic regularization in this paper are covariant, and the corresponding quantities in other coordinates can be given by coordinate transformations.

\section{Acknowledgements}

Y. Zhang is supported by NSFC Grant No. 11421303, 11675165, 11633001, 11961131007. The authors would like to thank A. Marciano for valuable discussions. 


\section{References}

[1] R. Utiyama, and B.S. DeWitt, J. Math. Phys. 3, 608 (1962).

[2] R.P. Feynman, and A.R. Hibbs, Quantum mechanics and path integration (McGraw-Hill,New York, 1965).

[3] P.J.E. Peebles, Principles of Physical Cosmology (Princeton University Press, Princeton, 1993).

[4] Y. Zhang, A\&A. 464, 811 (2007); Y. Zhang, and H.X. Miao, RAA. Vol.9 No.5, 501 (2009); Y. Zhang, and Q. Chen, A\&A. 581, A53 (2015); Y. Zhang, Q. Chen, S.G Wu, RAA. Vol.19 No.4, 53 (2019); B. Wang, and Y. Zhang, Phys. Rev. D 98, 103522 (2017); Phys. Rev. D 98, 123019 (2018); Phys. Rev. D 99, 123008 (2019); Y. Zhang, F. Qin, and B. Wang, Phys. Rev. D 98, 103523 (2017).

[5] C.P. Ma, and E. Bertschinger, Astrophys. J. 455, 7 (1995).

[6] W. Zhao, and Y. Zhang, Phys. Rev. D 74, 083006 (2006); T.Y. Xia, and Y. Zhang, Phys. Rev. D 78, 123005 (2008); Phys. Rev. D 79, 083002 (2009); Z. Cai, and Y. Zhang, Class. Quant. Grav. 29, 105009 (2012).

[7] P. Candelas, and D.J. Raine, Phys. Rev. D 12, 965 (1975).

[8] J.S. Dowker, and R. Critchley, Phys. Rev. D 13, 3224 (1976); Phys. Rev. D 16, 3390 (1977).

[9] L.S. Brown, Phys. Rev. D 15, 1469 (1977); L.S. Brown and J. P. Cassidy, Phys. Rev. D 15, 2810 (1977).

[10] T.S. Bunch, J. Phys. A: Math Gen. 12, 517 (1979).

[11] S.M. Christensen, Phys. Rev. D 14, 2490 (1976).

[12] S.M. Christensen, Phys. Rev. D 17, 946 (1978).

[13] T.S. Bunch, S.M. Christensen, and S.A. Fulling, Phys. Rev. D 18, 4435 (1978).

[14] T.S. Bunch, and P.C. W. Davies, Proc. R. Soc. Lond. A360, 117 (1978); Proc. R. Soc. Lond. A357, 381 (1977).

[15] R. Wald, Phys. Rev. D 17, 1477 (1978).

[16] S.L. Adler, J. Lieberman, and Y. J.Ng, Ann. Phys. (N.Y.) 106, 279 (1977).

[17] S. Hawking, Comm. math. Phys. 55, 133 (1977).

[18] K. Fujikawa, Phys. Rev. Lett. 44, 1733 (1980).

[19] J.S. Schwinger, Phys. Rev. 82, 664 (1951).

[20] S. DeWitt, Phys. Rep. 19C, 295 (1975).

[21] L. Parker, and S.A. Fulling, Phys. Rev. D 9, 341 (1974).

[22] S.A. Fulling, L. Parker, and B.L. Hu, Phys. Rev. D 10, 3905 (1974).

[23] B.L. Hu, and L. Parker, Phys. Rev. D 17, 933 (1978).

[24] B.L. Hu, Phys. Rev. D 18, 4460 (1978). 
[25] N. D. Birrell, Proc. R. Soc. Lond. B. 361, 513 (1978).

[26] T.S. Bunch, J. Phys. A: Math Gen. 11, 603 (1978).

[27] T.S. Bunch, and L. Parker, Phys. Rev. D 20, 2499 (1979).

[28] T.S. Bunch, J. Phys. A: Math Gen. 13, 1297 (1980).

[29] P.R. Anderson, and L. Parker, Phys. Rev. D 36, 2963 (1987).

[30] N. D. Birrell, and P. C. W. Davies, Quantum Fields in Curved Space (Cambridge University Press, Cambridge, 1982).

[31] L. Parker, and D. J. Toms, Quantum Field Theory in Curved Spacetime: Quantized Fields and Gravity (Cambridge University Press, Cambridge, 2009).

[32] L. Parker, arXiv:hep-th/0702216.

[33] T. Markkanen, and A. Tranberg, JCAP 08, 045 (2013).

[34] T. Markkanen, JCAP 05, 001 (2018).

[35] D.G. Wang, Y. Zhang, and J.W. Chen, Phys. Rev. D 94, 044033 (2016).

[36] Y. Zhang, and B. Wang, JCAP 11, 006 (2018).

[37] Y. Zhang, Phys. Lett. B 340, 18 (1994).

[38] G.N. Watson, Treatise on the theory of Bessel functions (Cambridge University Press, Cambridge, 1958).

[39] I.S. Gradshteyn, and I.M. Ryzhik, Tables of Integrals, Series, and Products (Academic Press, San Diego, 1980).

[40] F.W.J. Olver, D.W. Lozier, R.F. Boisvert, and C.W. Clark, NIST Handbook of Mathematical Functions (Cambridge University Press, Cambridge, 2010).

[41] B. Chakraborty, J. Math. Phys. 14, 188 (1973).

\section{A High $k$ expansions of exact modes}

In this appendix, we list some series expansions following from the analytical solution in de Sitter space which are used in the context.

In high $k$ limit, the mode $v_{k}(\tau)$ of $(9)$ approaches to

$$
\begin{aligned}
v_{k} \simeq & \frac{1}{\sqrt{2 k}} e^{i x}\left(1+i \frac{\left(4 \nu^{2}-1\right)}{8 x}-\frac{\left(16 \nu^{4}-40 \nu^{2}+9\right)}{128 x^{2}}-i \frac{\left(64 \nu^{6}-560 \nu^{4}+1036 \nu^{2}-225\right)}{3072 x^{3}}\right. \\
& +\frac{\left(256 \nu^{8}-5376 \nu^{6}+31584 \nu^{4}-51664 \nu^{2}+11025\right)}{98304 x^{4}} \\
& \left.-i \frac{\left(-1024 \nu^{10}+42240 \nu^{8}-561792 \nu^{6}+2764960 \nu^{4}-4228884 \nu^{2}+893025\right)}{3932160 x^{5}}+\ldots\right)
\end{aligned}
$$


with $x \equiv k|\tau|$, where the first term corresponds to the positive-frequency massless mode in Minkowski spacetime, and other terms are due to mass and expansion effects. Notice the pattern that $\nu^{2}$ goes with $1 / x$ in this high $k$ expansion. That is, the high $k$ expansion implies also an expansion at low $\frac{m^{2}}{H^{2}}$.

$$
\begin{aligned}
\left|v_{k}\right|^{2}= & \frac{1}{2 k}\left(1+\frac{4 \nu^{2}-1}{8 x^{2}}+\frac{3\left(4 \nu^{2}-1\right)\left(4 \nu^{2}-9\right)}{128 x^{4}}+\frac{5\left(4 \nu^{2}-1\right)\left(4 \nu^{2}-9\right)\left(4 \nu^{2}-25\right)}{1024 x^{6}}\right. \\
& \left.+\frac{35\left(4 \nu^{2}-1\right)\left(4 \nu^{2}-9\right)\left(4 \nu^{2}-25\right)\left(4 \nu^{2}-49\right)}{32768 x^{8}}+\ldots\right) .
\end{aligned}
$$

It can be also expressed in terms of $\omega$ for $\xi=0$ as follows

$$
\begin{aligned}
\left|v_{k}\right|^{2}= & \frac{1}{2 \omega}+\frac{-4 \nu^{2} \tau^{2}+4 \nu^{2}+9 \tau^{2}-1}{16 \tau^{2} \omega^{3}}+\frac{3\left(4 \nu^{2}-9\right)\left(4 \nu^{2} \tau^{4}-8 \nu^{2} \tau^{2}+4 \nu^{2}-9 \tau^{4}+2 \tau^{2}-1\right)}{256 \tau^{4} \omega^{5}} \\
& -\frac{5\left(4 \nu^{2}-9\right) A_{\tau}}{2048 \tau^{6} \omega^{7}}+\frac{35\left(4 \nu^{2}-9\right) B_{\tau}}{65536 \tau^{8} \omega^{9}}
\end{aligned}
$$

where

$$
\begin{aligned}
A_{\tau} \equiv & 16 \nu^{4} \tau^{6}-48 \nu^{4} \tau^{4}+48 \nu^{4} \tau^{2}-16 \nu^{4}-72 \nu^{2} \tau^{6}+120 \nu^{2} \tau^{4}-120 \nu^{2} \tau^{2} \\
& +104 \nu^{2}+81 \tau^{6}-27 \tau^{4}+27 \tau^{2}-25 \\
B_{\tau} \equiv & 64 \nu^{6} \tau^{8}-256 \nu^{6} \tau^{6}+384 \nu^{6} \tau^{4}-256 \nu^{6} \tau^{2}+64 \nu^{6}-432 \nu^{4} \tau^{8}+1216 \nu^{4} \tau^{6} \\
& -1824 \nu^{4} \tau^{4}+2240 \nu^{4} \tau^{2}-1200 \nu^{4}+972 \nu^{2} \tau^{8}-1584 \nu^{2} \tau^{6}+2376 \nu^{2} \tau^{4} \\
& -4144 \nu^{2} \tau^{2}+5196 \nu^{2}-729 \tau^{8}+324 \tau^{6}-486 \tau^{4}+900 \tau^{2}-1225 .
\end{aligned}
$$

In the context we shall plot all the graphs at a time $|\tau|=1$ for illustration, and the above is simplified as

$$
\begin{aligned}
\left|v_{k}\right|^{2}= & |\tau|\left(\frac{1}{2 \bar{\omega}}+\frac{1}{2 \bar{\omega}^{3}}-\frac{3\left(4 \nu^{2}-9\right)}{32 \bar{\omega}^{5}}-\frac{5\left(4 \nu^{2}-9\right)\left(4 \nu^{2}+7\right)}{256 \bar{\omega}^{7}}\right. \\
& \left.+\frac{35\left(4 \nu^{2}-9\right)\left(44 \nu^{2}-19\right)}{1024 \bar{\omega}^{9}}+\ldots\right)
\end{aligned}
$$

with $\bar{\omega} \equiv\left(k^{2}+\frac{m^{2}}{H^{2}}\right)^{1 / 2}$ which equals $\omega=\left(k^{2}+\frac{m^{2}}{H^{2} \tau^{2}}\right)^{1 / 2}$ at $|\tau|=1$. For $\xi=\frac{1}{6}$, (A.6) is replaced by the following

$$
\left|v_{k}\right|^{2}=|\tau|\left(\frac{1}{2 \bar{\omega}}+\frac{3\left(1-4 \nu^{2}\right)}{32 \bar{\omega}^{5}}+\frac{5\left(1-4 \nu^{2}\right)\left(4 \nu^{2}-25\right)}{256 \bar{\omega}^{7}}+\frac{105\left(4 \nu^{2}-1\right)\left(20 \nu^{2}-53\right)}{1024 \bar{\omega}^{9}}+\ldots\right) .
$$

The time derivative terms to the 4th adiabatic order are given by

$$
\begin{aligned}
& v_{k}^{\prime} \simeq-\sqrt{\frac{k}{2}} e^{i x}\left(i-\frac{4 \nu^{2}-1}{8 x}-i \frac{16 \nu^{4}+24 \nu^{2}-7}{128 x^{2}}+\frac{64 \nu^{6}+208 \nu^{4}-884 \nu^{2}+207}{3072 x^{3}}\right. \\
&\left.+i \frac{256 \nu^{8}+768 \nu^{6}-22176 \nu^{4}+47792 \nu^{2}-10575}{98304 x^{4}}+\ldots\right), \\
&\left|v_{k}^{\prime}\right|^{2}=k\left(\frac{1}{2}-\frac{\left(4 \nu^{2}-1\right)}{16 x^{2}}-\frac{\left(16 \nu^{4}-104 \nu^{2}+25\right)}{256 x^{4}}-\frac{\left(64 \nu^{6}-2096 \nu^{4}+4876 \nu^{2}-1089\right)}{2048 x^{6}}+\ldots\right),
\end{aligned}
$$


or, at $|\tau|=1$ for plotting,

$$
\begin{aligned}
\left|v_{k}^{\prime}\right|^{2}=k & \left(\frac{1}{2}+\frac{1-4 \nu^{2}}{16 \bar{\omega}^{2}}+\frac{48 \nu^{4}-56 \nu^{2}+11}{256 \bar{\omega}^{4}}+\frac{-320 \nu^{6}+2288 \nu^{4}-3900 \nu^{2}+837}{2048 \bar{\omega}^{6}}+\ldots\right), \\
\left(\frac{v_{k}}{a}\right)^{\prime}= & -\frac{H}{\sqrt{2 k}} e^{i x}\left(i x+\frac{\left(9-4 \nu^{4}\right)}{8}-i \frac{\left(16 \nu^{4}-40 \nu^{2}+9\right)}{128 x}+\frac{\left(4 \nu^{2}-9\right)\left(1-4 \nu^{2}\right)^{2}}{3072 x^{2}}\right. \\
& +i \frac{(2 \nu-5)(2 \nu-3)(2 \nu-1)(2 \nu+1)(2 \nu+3)(2 \nu+5)\left(4 \nu^{2}+15\right)}{98304 x^{3}} \\
& \left.-\frac{(2 \nu-7)(2 \nu-5)(2 \nu-3)(2 \nu-1)(2 \nu+1)(2 \nu+3)(2 \nu+5)(2 \nu+7)\left(4 \nu^{2}+39\right)}{3932160 x^{4}}+\ldots\right) \\
\left|\left(\frac{v_{k}}{a}\right)^{\prime}\right|^{2} & =\frac{\pi H^{2}}{4 k}\left|\frac{d}{d x}\left(x^{3 / 2} H_{\nu}^{(2)}(x)\right)\right|^{2} \\
& =H^{2}\left(\frac{x^{2}}{2 k}+\frac{9-4 \nu^{2}}{16 k}-\frac{16 \nu^{4}-40 \nu^{2}+9}{256 k x^{2}}+\frac{441-1996 \nu^{2}+944 \nu^{4}-64 \nu^{6}}{2048 k x^{4}}+\ldots\right) .
\end{aligned}
$$

In low $k$ limit,

$$
\begin{gathered}
v_{k} \simeq\left(\frac{x}{2}\right)^{-\nu+\frac{1}{2}} \frac{\Gamma(\nu)}{\sqrt{2 \pi k}} e^{i \frac{\pi}{2}\left(\nu-\frac{1}{2}\right)}, \\
\left|v_{k}\right|^{2} \simeq x^{-2 \nu}|\tau| \frac{2^{2 \nu-2} \Gamma(\nu)^{2}}{\pi}, \\
\left|\frac{v_{k}}{a}\right|^{2} \simeq H^{2} x^{-2 \nu}|\tau|^{3} \frac{2^{2 \nu-2} \Gamma(\nu)^{2}}{\pi},
\end{gathered}
$$

and the time derivatives are

$$
\begin{gathered}
v_{k}^{\prime} \simeq-\left(\frac{x}{2}\right)^{-\nu-\frac{1}{2}} k^{1 / 2} \frac{\Gamma(\nu)-2 \Gamma(\nu+1)}{4 \sqrt{2 \pi}} e^{i \frac{\pi}{2}\left(\nu-\frac{1}{2}\right)}, \\
\left|v_{k}^{\prime}\right|^{2} \simeq\left(\frac{x}{2}\right)^{-2 \nu-1} k \frac{(\Gamma(\nu)-2 \Gamma(\nu+1))^{2}}{32 \pi}, \\
\left(\frac{v_{k}}{a}\right)^{\prime}=-H\left(\frac{x}{2}\right)^{-\nu+\frac{1}{2}} k^{-\frac{1}{2}} \frac{(3 \Gamma(\nu)-2 \Gamma(\nu+1))}{2 \sqrt{2 \pi}} e^{i \frac{\pi}{2}\left(\nu-\frac{1}{2}\right)}, \\
\left|\left(\frac{v_{k}}{a}\right)^{\prime}\right|^{2}=H^{2} x^{-2 \nu}|\tau| \frac{2^{2 \nu}(3 \Gamma(\nu)-2 \Gamma(\nu+1))^{2}}{16 \pi} .
\end{gathered}
$$

In these expressions the index $\nu$ is for a general $\xi$. 


\section{B The WKB modes}

The WKB approximation $[21,22,28,29,41]$ to the solution of (5) in a flat RW spacetime is written as the following

$$
v_{k}^{(n)}(\tau)=\left(2 W_{k}(\tau)\right)^{-1 / 2} \exp \left[-i \int^{\tau} W_{k}\left(\tau^{\prime}\right) d \tau^{\prime}\right]
$$

where the effective frequency is

$$
\left(W_{k}(\tau)\right)=\left[k^{2}+m^{2} a^{2}+\left(\xi-\frac{1}{6}\right) a^{2} R-\frac{1}{2}\left(\frac{W_{k}^{\prime \prime}}{W_{k}}-\frac{3}{2}\left(\frac{W_{k}^{\prime}}{W_{k}}\right)^{2}\right)\right]^{1 / 2} .
$$

The WKB solution of $W_{k}$ is obtained by iteratively solving (B.2) to a desired adiabatic order. Take the 0th-order effective frequency [28],

$$
W_{k}^{(0)}=\omega=\sqrt{k^{2}+m^{2} a^{2}},
$$

and the 0th-order adiabatic mode

$$
v_{k}^{(0)}(\tau)=(2 \omega)^{-1 / 2} e^{-i \int^{\tau} \omega d \tau^{\prime}}
$$

The 0th-order quantities that appear in the 0th-order subtraction terms are

$$
\begin{gathered}
\left|v_{k}^{(0)}\right|^{2}=\frac{1}{2 W_{k}^{(0)}}=\frac{1}{2 \omega}, \\
\left|v_{k}^{(0) \prime}\right|^{2}=\frac{1}{2}\left(\frac{\left(W_{k}^{(0)^{\prime}}\right)^{2}}{4\left(W_{k}^{(0)}\right)^{3}}+W_{k}^{(0)}\right) \simeq \frac{\omega}{2}, \\
v_{k}^{(0) \prime} v_{k}^{(0) *}+v_{k}^{(0)} v_{k}^{(0) * \prime} \simeq 0 .
\end{gathered}
$$

The 2nd-order adiabatic mode

$$
v_{k}^{(2)}(\tau)=\left(2 W_{k}^{(2)}(\tau)\right)^{-1 / 2} e^{-i \int^{\tau} W_{k}^{(2)}\left(\tau^{\prime}\right) d \tau^{\prime}},
$$

The first iteration of (B.2) is

$$
W_{k}^{(2)}=\left[k^{2}+m^{2} a^{2}+\left(\xi-\frac{1}{6}\right) a^{2} R-\frac{1}{2}\left(\frac{W_{k}^{(0) \prime \prime}}{W_{k}^{(0)}}-\frac{3}{2}\left(\frac{W_{k}^{(0) \prime}}{W_{k}^{(0)}}\right)^{2}\right)\right]^{\frac{1}{2}}
$$

By

$$
\left(\xi-\frac{1}{6}\right) a^{2} R-\frac{1}{2}\left(\frac{W_{k}^{(0) \prime \prime}}{W_{k}^{(0)}}-\frac{3}{2}\left(\frac{W_{k}^{(0) \prime}}{W_{k}^{(0)}}\right)^{2}\right)=6\left(\xi-\frac{1}{6}\right) \frac{a^{\prime \prime}}{a}-\frac{m^{2}\left(a a^{\prime \prime}+a^{\prime 2}\right)}{2 \omega^{2}}+\frac{5 m^{4} a^{2} a^{\prime 2}}{4 \omega^{4}},
$$

keeping only up to two time-derivatives of metric, $a^{\prime \prime}$ and $a^{2}$, gives the 2 nd-order effective frequency,

$$
\begin{gathered}
W_{k}^{(2)} \simeq \omega+3\left(\xi-\frac{1}{6}\right) \frac{1}{\omega} \frac{a^{\prime \prime}}{a}-\frac{m^{2}\left(a^{\prime \prime} a+a^{\prime 2}\right)}{4 \omega^{3}}+\frac{5 m^{4} a^{\prime 2} a^{2}}{8 \omega^{5}} \\
\left(W_{k}^{(2)}\right)^{-1} \simeq \frac{1}{\omega}-3\left(\xi-\frac{1}{6}\right) \frac{a^{\prime \prime} / a}{\omega^{3}}+\frac{m^{2}\left(a^{\prime \prime} a+a^{\prime 2}\right)}{4 \omega^{5}}-\frac{5 m^{4} a^{\prime 2} a^{2}}{8 \omega^{7}} .
\end{gathered}
$$


It should be observed that $W_{k}^{(2)}$ actually contains four terms up to $\omega^{-5}$, and $\left(W_{k}^{(2)}\right)^{-1}$ has up to $\omega^{-7}$, even though retaining only two time-derivatives. The 2nd-order subtraction term for the power spectrum uses

$$
\left|v_{k}^{(2)}\right|^{2}=\left(2 W_{k}^{(2)}\right)^{-1}
$$

which is given by (B.10). The 2nd-order subtraction term for $\rho_{k}$ has threes terms, its $k^{2}$ and mass terms are $\propto\left|v_{k}^{(2)}\right|^{2}$, and its time derivative term is proportional to

$$
\left|\left(\frac{v_{k}^{(2)}}{a}\right)^{\prime}\right|^{2}=\frac{1}{a^{2}}\left(\left|v_{k}^{(2) \prime}\right|^{2}-\frac{a^{\prime}}{a}\left(v_{k}^{(2) \prime} v_{k}^{(2) *}+v_{k}^{(2)} v_{k}^{(2) * \prime}\right)+\left(\frac{a^{\prime}}{a}\right)^{2}\left|v_{k}^{(2)}\right|^{2}\right) .
$$

By

$$
v_{k}^{(2)^{\prime}}(\tau)=\frac{1}{\sqrt{2}}\left(\frac{-W_{k}^{(2)^{\prime}}}{2\left(W_{k}^{(2)}\right)^{3 / 2}}-i \frac{W_{k}^{(2)}}{\sqrt{W_{k}^{(2)}}}\right) e^{-i \int^{\tau} W_{k}^{(2)} d \tau^{\prime}}
$$

one has up to the 2 nd adiabatic order $W_{k}^{(2)^{\prime}} \simeq \omega^{\prime}=\frac{m^{2} a^{2}}{\omega} \frac{a^{\prime}}{a}$,

$$
\begin{aligned}
&\left|v_{k}^{(2) \prime}\right|^{2}=\frac{1}{2}\left(\frac{\left(W_{k}^{(2)^{\prime}}\right)^{2}}{4\left(W_{k}^{(2)}\right)^{3}}+W_{k}^{(2)}\right)=\frac{\omega}{2}+\frac{7 m^{4} a^{2} a^{\prime 2}}{16 \omega^{5}}-\frac{m^{2}\left(a a^{\prime \prime}+a^{\prime 2}\right)}{8 \omega^{3}}+\left(\xi-\frac{1}{6}\right) \frac{3 a^{\prime \prime}}{2 \omega a} \\
& v_{k}^{(2) \prime} v_{k}^{(2) *}+v_{k}^{(2)} v_{k}^{(2) * \prime}=-\left(\frac{W_{k}^{(2)^{\prime}}}{2 W_{k}^{(2)}}+i W_{k}^{(2)}\right)\left(2 W_{k}^{(2)}\right)^{-1}-\left(\frac{W_{k}^{(2)^{\prime}}}{2 W_{k}^{(2)}}-i W_{k}^{(2)}\right)\left(2 W_{k}^{(2)}\right)^{-1} \\
&=-\frac{W_{k}^{(2)^{\prime}}}{2\left(W_{k}^{(2)}\right)^{2}}=-\frac{m^{2} a a^{\prime}}{2 \omega^{3}} .
\end{aligned}
$$

Putting together, one has

$$
\left|\left(\frac{v_{k}^{(2)}}{a}\right)^{\prime}\right|^{2}=\frac{1}{a^{2}}\left[\frac{\omega}{2}+\frac{1}{2 \omega} \frac{a^{\prime 2}}{a^{2}}+\frac{m^{2}\left(3 a^{\prime 2}-a a^{\prime \prime}\right)}{8 \omega^{3}}+\frac{7 m^{4} a^{2} a^{\prime 2}}{16 \omega^{5}}+\left(\xi-\frac{1}{6}\right) \frac{3 a^{\prime \prime}}{2 \omega a}\right] .
$$

Similarly, the 4th-order adiabatic mode

$$
v_{k}^{(4)}(\tau)=\left(2 W_{k}^{(4)}(\tau)\right)^{-1 / 2} e^{-i \int^{\tau} W_{k}^{(4)}\left(\tau^{\prime}\right) d \tau^{\prime}},
$$

the 4th-order effective frequency is derived by iteration

$$
W_{k}^{(4)}=\left[k^{2}+m^{2} a^{2}+\left(\xi-\frac{1}{6}\right) a^{2} R-\frac{1}{2}\left(\frac{W_{k}^{(2) \prime \prime}}{W_{k}^{(2)}}-\frac{3}{2}\left(\frac{W_{k}^{(2) \prime}}{W_{k}^{(2)}}\right)^{2}\right)\right]^{\frac{1}{2}} .
$$

Dropping terms of five time derivatives, one obtains

$$
\begin{aligned}
W_{k}^{(4)}= & \omega+\left(\xi-\frac{1}{6}\right) \frac{3 a^{\prime \prime}}{a \omega}-\frac{m^{2}\left(a^{\prime \prime} a+a^{2}\right)}{4 \omega^{3}}+\frac{5 m^{4} a^{2} a^{2}}{8 \omega^{5}} \\
& +\frac{m^{2}\left(a^{\prime \prime \prime \prime} a+4 a^{\prime \prime \prime} a^{\prime}+3 a^{\prime \prime} a^{\prime \prime}\right)}{16 \omega^{5}}-\frac{m^{4}\left(19 a^{2} a^{\prime \prime 2}+19 a^{4}+28 a^{2} a^{\prime \prime \prime} a^{\prime}+122 a a^{\prime 2} a^{\prime \prime}\right)}{32 \omega^{7}} \\
& +\frac{221 m^{6}\left(a^{\prime \prime} a^{\prime 2} a^{3}+a^{\prime 4} a^{2}\right)}{32 \omega^{9}}-\frac{1105 m^{8} a^{4} a^{\prime 4}}{128 \omega^{11}}
\end{aligned}
$$




$$
\begin{aligned}
& +\left(\xi-\frac{1}{6}\right)\left[-\frac{3}{4 \omega^{3}}\left(\frac{a^{\prime \prime \prime \prime}}{a}-\frac{a^{\prime \prime 2}}{a^{2}}-\frac{2 a^{\prime \prime \prime} a^{\prime}}{a^{2}}+\frac{2 a^{\prime 2} a^{\prime \prime}}{a^{3}}\right)\right. \\
& \left.+\frac{m^{2}}{4 \omega^{5}}\left(15 a^{\prime} a^{\prime \prime \prime}-6 \frac{a^{\prime 2} a^{\prime \prime}}{a}+9 a^{\prime \prime} a^{\prime \prime}\right)-\frac{75 m^{4} a a^{\prime 2} a^{\prime \prime}}{8 \omega^{7}}\right]-\frac{9}{2}\left(\xi-\frac{1}{6}\right)^{2} \frac{a^{\prime \prime 2}}{a^{2} \omega^{3}}
\end{aligned}
$$

which is the same as Bunch's (2.20) [28], and

$$
\begin{aligned}
\left(W_{k}^{(4)}\right)^{-1}= & \frac{1}{\omega}+\frac{m^{2}\left(a a^{\prime \prime}+a^{2}\right)}{4 \omega^{5}}-\frac{5 m^{4} a^{2} a^{2}}{8 \omega^{7}}-3\left(\xi-\frac{1}{6}\right) \frac{1}{\omega^{3}} \frac{a^{\prime \prime}}{a} \\
& -\frac{m^{2}\left(3 a^{\prime \prime 2}+a^{\prime \prime \prime \prime} a+4 a^{\prime \prime \prime} a^{\prime}\right)}{16 \omega^{7}}+\frac{7 m^{4}\left(3 a^{2} a^{\prime \prime 2}+3 a^{\prime 4}+18 a a^{2} a^{\prime \prime}+4 a^{2} a^{\prime \prime \prime} a^{\prime}\right)}{32 \omega^{9}} \\
& -\frac{231 m^{6} a^{2}\left(a^{\prime 4}+a a^{\prime 2} a^{\prime \prime}\right)}{32 \omega^{11}}+\frac{1155 m^{8} a^{4} a^{\prime 4}}{128 \omega^{13}} \\
& +\left(\xi-\frac{1}{6}\right)\left[\frac{3}{4 \omega^{5}}\left(-\frac{a^{\prime \prime}}{a^{2}}+\frac{a^{\prime \prime \prime \prime}}{a}+2 \frac{a^{\prime 2} a^{\prime \prime}}{a^{3}}-2 \frac{a^{\prime \prime \prime} a^{\prime}}{a^{2}}\right)\right. \\
& \left.-\frac{15 m^{2}\left(a^{\prime \prime 2}+a^{\prime \prime \prime} a^{\prime}\right)}{4 \omega^{7}}+\frac{105 m^{4} a a^{\prime 2} a^{\prime \prime}}{8 \omega^{9}}\right]+\left(\xi-\frac{1}{6}\right)^{2} \frac{27}{2 \omega^{5}} \frac{a^{\prime \prime 2}}{a^{2}} .
\end{aligned}
$$

Keeping only up to second order time derivative terms, $W_{k}^{(4)}$ and $\left(W_{k}^{(4)}\right)^{-1}$ reduce to the 2 nd-order expressions $W_{k}^{(2)}$ and $\left(W_{k}^{(2)}\right)^{-1}$ in (B.9) and (B.10), respectively. Notice that for the special case $\xi=\frac{1}{6}$ and $m=0$, we have

$$
W_{k}^{(4)}=W_{k}^{(2)}=W_{k}^{(0)}=k,
$$

which holds for a general RW spacetime, also implies Eq.(117) in Sect 4 for de Sitter space.

From the effective frequency $W_{k}^{(4)}$ in (B.19) and its reciprocal (B.20) we can calculate the following quantities that occur in the 4th-order subtraction terms for the power spectrum and spectral stress tensor.

$$
\begin{aligned}
\left|v_{k}^{(4) \prime}\right|^{2}= & \frac{1}{2}\left(\frac{\left(W_{k}^{(4)^{\prime}}\right)^{2}}{4\left(W_{k}^{(4)}\right)^{3}}+W_{k}^{(4)}\right) \\
= & \frac{\omega}{2}+\frac{7 m^{4} a^{2} a^{\prime 2}}{16 \omega^{5}}-\frac{m^{2}\left(a a^{\prime \prime}+a^{\prime 2}\right)}{8 \omega^{3}}+\left(\xi-\frac{1}{6}\right) \frac{3 a^{\prime \prime}}{2 \omega a}+\frac{m^{2}}{64 \omega^{5}}\left(2 a a^{\prime \prime \prime \prime}+6 a^{\prime \prime 2}+8 a^{\prime} a^{\prime \prime \prime}\right) \\
& -\frac{m^{4}}{256 \omega^{7}}\left(76 a^{\prime 4}+536 a a^{\prime 2} a^{\prime \prime}+76 a^{2} a^{\prime \prime 2}+128 a^{2} a^{\prime} a^{\prime \prime \prime}\right) \\
& +\frac{259 m^{6}}{512 \omega^{9}}\left(8 a^{2} a^{\prime 4}+8 a^{3} a^{\prime 2} a^{\prime \prime}\right)-\frac{1365 m^{8} a^{4} a^{\prime 4}}{256 \omega^{11}} \\
& +\left(\xi-\frac{1}{6}\right)\left[-\frac{1}{16 \omega^{3}}\left(\frac{6 a^{\prime \prime \prime \prime}}{a}+\frac{12 a^{\prime 2} a^{\prime \prime}}{a^{3}}-\frac{6 a^{\prime \prime 2}}{a^{2}}-\frac{12 a^{\prime} a^{\prime \prime \prime}}{a^{2}}\right)\right. \\
& \left.+\frac{m^{2} a^{2}}{64 \omega^{5}}\left(-\frac{96 a^{\prime 2} a^{\prime \prime}}{a^{3}}+\frac{72 a^{\prime \prime 2}}{a^{2}}+\frac{168 a^{\prime} a^{\prime \prime \prime}}{a^{2}}\right)-\frac{105 m^{4} a a^{\prime 2} a^{\prime \prime}}{16 \omega^{7}}\right]-\left(\xi-\frac{1}{6}\right)^{2} \frac{9}{4 \omega^{3}} \frac{a^{\prime \prime 2}}{a^{2}}, \\
& \\
v_{k}^{(4) \prime} v_{k}^{(4) *}+ & v_{k}^{(4)} v_{k}^{(4) * \prime}=\frac{-}{2\left(W_{k}^{(4)}\right)^{2}} \\
& +\frac{m^{2} a a^{\prime}}{2 \omega^{3}}+\frac{m^{2}\left(3 a^{\prime} a^{\prime \prime}+a a^{\prime \prime \prime}\right)}{8 \omega^{5}}-\frac{5 m^{4}\left(a a^{\prime 3}+a^{2} a^{\prime} a^{\prime \prime}\right)}{4 \omega^{7}}+\frac{35 m^{6} a^{3} a^{\prime 3}}{16 \omega^{9}}
\end{aligned}
$$


putting together yields

$$
\begin{aligned}
\left|\left(\frac{v_{k}^{(4)}}{a}\right)^{\prime}\right|^{2}= & \frac{1}{a^{2}}\left[\left|v_{k}^{(4)^{\prime}}\right|^{2}-\frac{a^{\prime}}{a}\left(v_{k}^{(4)^{\prime}} v_{k}^{(4) *}+v_{k}^{(4)} v_{k}^{(4) *^{\prime}}\right)+\left(\frac{a^{\prime}}{a}\right)^{2}\left|v_{k}^{(4)}\right|^{2}\right] \\
= & \frac{1}{a^{2}}\left[\frac{\omega}{2}+\frac{1}{2 \omega} \frac{a^{\prime 2}}{a^{2}}+\frac{m^{2}\left(3 a^{\prime 2}-a a^{\prime \prime}\right)}{8 \omega^{3}}+\frac{7 m^{4} a^{2} a^{\prime 2}}{16 \omega^{5}}+\left(\xi-\frac{1}{6}\right) \frac{3 a^{\prime \prime}}{2 \omega a}\right. \\
& +\frac{m^{2}\left(a^{3} a^{\prime \prime \prime \prime}+3 a^{\prime \prime 2} a^{2}+4 a^{\prime 4}-8 a a^{\prime 2} a^{\prime \prime}\right)}{32 \omega^{5} a^{2}}+\frac{m^{4}\left(41 a^{\prime 4}-54 a a^{\prime 2} a^{\prime \prime}-19 a^{2} a^{\prime \prime 2}-32 a^{2} a^{\prime} a^{\prime \prime \prime}\right)}{64 \omega^{7}} \\
& +\frac{m^{6}\left(119 a^{2} a^{\prime 4}+259 a^{3} a^{\prime 2} a^{\prime \prime}\right)}{64 \omega^{9}}-\frac{1365 a^{4} m^{8} a^{\prime 4}}{256 \omega^{11}} \\
& -\left(\xi-\frac{1}{6}\right)\left(\frac{1}{8 \omega^{3}}\left(\frac{3 a^{\prime \prime \prime \prime}}{a}-\frac{18 a^{\prime} a^{\prime \prime \prime}}{a^{2}}+\frac{30 a^{\prime 2} a^{\prime \prime}}{a^{3}}-\frac{3 a^{\prime \prime 2}}{a^{2}}\right)+\frac{m^{2}}{8 \omega^{5} a}\left(48 a^{\prime 2} a^{\prime \prime}-9 a a^{\prime \prime 2}-21 a a^{\prime} a^{\prime \prime \prime}\right)\right. \\
& \left.\left.+\frac{105 m^{4} a a^{\prime 2} a^{\prime \prime}}{16 \omega^{7}}\right)-\left(\xi-\frac{1}{6}\right)^{2} \frac{9}{4 \omega^{3}} \frac{a^{\prime \prime 2}}{a^{2}}\right] .
\end{aligned}
$$

So far the formulae given above are valid for a general flat RW spacetime.

For de Sitter space, the scale factor satisfies the following relation

$$
\left(-\frac{1}{6}\right)\left[\frac{3}{4 \omega^{5}}\left(-\frac{a^{\prime \prime 2}}{a^{2}}+\frac{a^{\prime \prime \prime \prime}}{a}+2 \frac{a^{\prime 2} a^{\prime \prime}}{a^{3}}-2 \frac{a^{\prime \prime \prime} a^{\prime}}{a^{2}}\right)\right]+\left(-\frac{1}{6}\right)^{2} \frac{27}{2 \omega^{5}} \frac{a^{\prime \prime 2}}{a^{2}}=0 .
$$

By this relation, in the special case $\xi=0$ and $m=0$, we find that

$$
W_{k}^{(4)}=W_{k}^{(2)}, \quad\left(W_{k}^{(4)}\right)^{-1}=\left(W_{k}^{(2)}\right)^{-1}
$$

which imply Eq.(77) in Sect 3.

\section{The subtraction terms for the stress tensor}

Substitution of the 0th-order adiabatic mode $v_{k}^{(0)}$ of (B.4) into (19) (21) in place of $v_{k}$ yields the 0th-order subtraction term for stress tensor

$$
\begin{aligned}
\rho_{k A 0}= & \frac{k^{3}}{4 \pi^{2} a^{4}}\left[\left|v_{k}^{(0) \prime}\right|^{2}+k^{2}\left|v_{k}^{(0)}\right|^{2}+m^{2} a^{2}\left|v_{k}^{(0)}\right|^{2}\right. \\
& \left.+(6 \xi-1)\left(\frac{a^{\prime}}{a}\left(v_{k}^{(0) \prime} v_{k}^{(0) *}+v_{k}^{(0)} v_{k}^{(0) * \prime}\right)-\frac{a^{\prime 2}}{a^{2}}\left|v_{k}^{(0)}\right|^{2}\right)\right] \\
\simeq & \frac{k^{3}}{4 \pi^{2} a^{4}} \omega, \\
p_{k A 0}= & \frac{k^{3}}{4 \pi^{2} a^{4}}\left[\frac{1}{3}\left|v_{k}^{(0) \prime}\right|^{2}+\frac{1}{3} k^{2}\left|v_{k}^{(0)}\right|^{2}-\frac{1}{3} m^{2} a^{2}\left|v_{k}^{(0)}\right|^{2}\right. \\
& +2\left(\xi-\frac{1}{6}\right)\left(-2\left|v_{k}^{(0) \prime}\right|^{2}+3 \frac{a^{\prime}}{a}\left(v_{k}^{(0) \prime} v^{(0) *}{ }_{k}^{*}+v_{k}^{(0)} v^{(0) * \prime}\right)\right. \\
& \left.\left.-3\left(\frac{a^{\prime}}{a}\right)^{2}\left|v_{k}^{(0)}\right|^{2}+2\left(k^{2}+m^{2} a^{2}\right)\left|v_{k}^{(0)}\right|^{2}+12 \xi \frac{a^{\prime \prime}}{a}\left|v_{k}^{(0)}\right|^{2}\right)\right] \\
\simeq & \frac{k^{3}}{12 \pi^{2} a^{4}}\left[\omega-\frac{m^{2} a^{2}}{\omega}\right],
\end{aligned}
$$




$$
\left\langle T_{\mu}^{\mu}\right\rangle_{k A 0}=\rho_{k A 0}-3 p_{k A 0}=\frac{m^{2} k^{3}}{2 \pi^{2} a^{2}} \frac{1}{2 \omega} .
$$

Note that these contain no time derivatives of $a(\tau)$, and do not depend on the coupling $\xi$.

Substitution of the 2nd-order adiabatic mode $v_{k}^{(2)}$ of (B.7) into (19) (21) in place of $v_{k}$ yields the 2nd-order subtraction term for the spectral energy density and pressure

$$
\begin{aligned}
\rho_{k A 2} & =\frac{k^{3}}{4 \pi^{2} a^{4}}\left[\omega+\frac{m^{4} a^{4}}{8 \omega^{5}} \frac{a^{\prime 2}}{a^{2}}+\left(\xi-\frac{1}{6}\right)\left(-\frac{3}{\omega} \frac{a^{\prime 2}}{a^{2}}-\frac{3 m^{2} a^{\prime 2}}{\omega^{3}}\right)\right], \\
p_{k A 2}= & \frac{k^{3}}{12 \pi^{2} a^{4}}\left[\omega-\frac{m^{2} a^{2}}{\omega}-\frac{m^{4} a^{4}}{8 \omega^{5}}\left(\frac{2 a^{\prime \prime}}{a}+\frac{a^{\prime 2}}{a^{2}}\right)+\frac{5 m^{6} a^{6}}{8 \omega^{7}} \frac{a^{\prime 2}}{a^{2}}\right. \\
& \left.+\left(\xi-\frac{1}{6}\right)\left(\frac{1}{\omega}\left(6 \frac{a^{\prime \prime}}{a}-9 \frac{a^{\prime 2}}{a^{2}}\right)+\frac{6 m^{2} a^{2}}{\omega^{3}}\left(\frac{a^{\prime \prime}}{a}-\frac{a^{\prime 2}}{a^{2}}\right)-\frac{9 m^{4} a^{4}}{\omega^{5}} \frac{a^{\prime 2}}{a^{2}}\right)\right] .
\end{aligned}
$$

They reduce to (C.1) (C.2) when the time derivatives of $a(\tau)$ are dropped.

Substitution of the 4 th-order adiabatic mode $v_{k}^{(4)}$ into (19) (21) in place of $v_{k}$, yields the 4 th-order subtraction term for spectral energy density and pressure $[28,29]$

$$
\begin{aligned}
& \rho_{k A 4}=\frac{k^{3}}{4 \pi^{2} a^{4}}\left[\omega+\frac{m^{4} a^{4}}{8 \omega^{5}} \frac{a^{\prime 2}}{a^{2}}-\frac{m^{4} a^{4}}{32 \omega^{7}}\left(2 \frac{a^{\prime \prime \prime} a^{\prime}}{a^{2}}-\frac{a^{\prime \prime 2}}{a^{2}}+\frac{4 a^{\prime \prime} a^{\prime 2}}{a^{3}}-\frac{a^{\prime 4}}{a^{4}}\right)\right. \\
& +\frac{7 m^{6} a^{6}}{16 \omega^{9}}\left(\frac{a^{\prime \prime} a^{\prime 2}}{a^{3}}+\frac{a^{\prime 4}}{a^{4}}\right)-\frac{105 m^{8} a^{8}}{128 \omega^{11}} \frac{a^{4}}{a^{4}} \\
& +\left(\xi-\frac{1}{6}\right)\left[-\frac{3}{\omega} \frac{a^{\prime 2}}{a^{2}}-\frac{3 m^{2} a^{\prime 2}}{\omega^{3}}+\frac{3 m^{2} a^{2}}{4 \omega^{5}}\left(\frac{2 a^{\prime \prime \prime} a^{\prime}}{a^{2}}-\frac{a^{\prime \prime 2}}{a^{2}}-\frac{a^{\prime 4}}{a^{4}}\right)\right. \\
& \left.-\frac{15 m^{4} a^{4}}{8 \omega^{7}}\left(\frac{4 a^{\prime \prime} a^{\prime 2}}{a^{3}}+\frac{3 a^{\prime 4}}{a^{4}}\right)+\frac{105 m^{6} a^{6}}{8 \omega^{9}} \frac{a^{\prime 4}}{a^{4}}\right] \\
& \left.+\left(\xi-\frac{1}{6}\right)^{2}\left[-\frac{9}{2 \omega^{3}}\left(\frac{2 a^{\prime \prime \prime} a^{\prime}}{a^{2}}-\frac{a^{\prime \prime 2}}{a^{2}}-\frac{4 a^{\prime \prime} a^{\prime 2}}{a^{3}}\right)+\frac{27 m^{2} a^{2}}{\omega^{5}} \frac{a^{\prime \prime} a^{\prime 2}}{a^{3}}\right]\right], \\
& p_{k A 4}=\frac{1}{3}\left(\frac{k^{3}}{4 \pi^{2} a^{4}}\right)\left[\omega-\frac{m^{2} a^{2}}{\omega}-\frac{m^{4} a^{4}}{8 \omega^{5}}\left(\frac{2 a^{\prime \prime}}{a}+\frac{a^{\prime 2}}{a^{2}}\right)\right. \\
& +\frac{5 m^{6} a^{6}}{8 \omega^{7}} \frac{a^{\prime 2}}{a^{2}}+\frac{m^{4} a^{4}}{32 \omega^{7}}\left(\frac{2 a^{\prime \prime \prime \prime}}{a}+6 \frac{a^{\prime \prime \prime} a^{\prime}}{a^{2}}+7 \frac{a^{\prime \prime 2}}{a^{2}}-\frac{4 a^{\prime \prime} a^{\prime 2}}{a^{3}}+\frac{a^{\prime 4}}{a^{4}}\right) \\
& -\frac{m^{6} a^{6}}{32 \omega^{9}}\left(\frac{28 a^{\prime \prime \prime} a^{\prime}}{a^{2}}+21 \frac{a^{\prime \prime 2}}{a^{2}}+\frac{112 a^{\prime \prime} a^{\prime 2}}{a^{3}}+7 \frac{a^{\prime 4}}{a^{4}}\right) \\
& +\frac{21 m^{8} a^{8}}{128 \omega^{11}}\left(44 \frac{a^{\prime \prime} a^{\prime 2}}{a^{3}}+39 \frac{a^{4}}{a^{4}}\right)-\frac{1155 m^{10} a^{10}}{128 \omega^{13}} \frac{a^{\prime 4}}{a^{4}} \\
& +\left(\xi-\frac{1}{6}\right)\left(\frac{1}{\omega}\left(6 \frac{a^{\prime \prime}}{a}-9 \frac{a^{\prime 2}}{a^{2}}\right)+\frac{6 m^{2} a^{2}}{\omega^{3}}\left(\frac{a^{\prime \prime}}{a}-\frac{a^{\prime 2}}{a^{2}}\right)\right. \\
& +\frac{3 m^{2} a^{2}}{4 \omega^{5}}\left(-\frac{2 a^{\prime \prime \prime \prime}}{a}+\frac{2 a^{\prime \prime \prime} a^{\prime}}{a^{2}}+\frac{4 a^{\prime \prime} a^{\prime 2}}{a^{3}}-\frac{a^{\prime \prime 2}}{a^{2}}-\frac{3 a^{\prime 4}}{a^{4}}\right)-\frac{9 m^{4} a^{4}}{\omega^{5}} \frac{a^{\prime 2}}{a^{2}} \\
& +\frac{15 m^{4} a^{4}}{8 \omega^{7}}\left(\frac{8 a^{\prime \prime \prime} a^{\prime}}{a^{2}}+\frac{6 a^{\prime \prime 2}}{a^{2}}+\frac{12 a^{\prime \prime} a^{\prime 2}}{a^{3}}-\frac{5 a^{\prime 4}}{a^{4}}\right) \\
& \left.-\frac{105 m^{6} a^{6}}{2 \omega^{9}}\left(\frac{2 a^{\prime \prime} a^{\prime 2}}{a^{3}}+\frac{a^{\prime 4}}{a^{4}}\right)+\frac{945 m^{8} a^{8}}{8 \omega^{11}} \frac{a^{\prime 4}}{a^{4}}\right)
\end{aligned}
$$




$$
\begin{aligned}
& +\left(\xi-\frac{1}{6}\right)^{2}\left(\frac{9}{2 \omega^{3}}\left(\frac{2 a^{\prime \prime \prime \prime}}{a}-\frac{10 a^{\prime \prime \prime} a^{\prime}}{a^{2}}-\frac{5 a^{\prime \prime 2}}{a^{2}}+\frac{16 a^{\prime \prime} a^{\prime 2}}{a^{3}}\right)\right. \\
& \left.\left.-\frac{27 m^{2} a^{2}}{2 \omega^{5}}\left(\frac{4 a^{\prime \prime \prime} a^{\prime}}{a^{2}}+\frac{3 a^{\prime \prime 2}}{a^{2}}-\frac{8 a^{\prime \prime} a^{\prime 2}}{a^{3}}\right)+\frac{135 m^{4} a^{4}}{\omega^{7}} \frac{a^{\prime \prime} a^{2}}{a^{3}}\right)\right] .
\end{aligned}
$$

They reduce to (C.4) (C.5) when the fourth order time derivatives of $a(\tau)$ are dropped. The subtraction term of the spectral trace is given by

$$
\begin{aligned}
\left\langle T_{\mu}^{\mu}\right\rangle_{k A 4}= & \rho_{k A 4}-3 p_{k A 4} \\
= & \frac{k^{3}}{4 \pi^{2} a^{4}}\left[\frac{m^{2} a^{2}}{\omega}+\frac{m^{4} a^{4}}{4 \omega^{5}}\left(\frac{a^{\prime \prime}}{a}+\frac{a^{\prime 2}}{a^{2}}\right)-\frac{5 m^{6} a^{6} a^{\prime 2}}{8 \omega^{7}} \frac{m^{4} a^{4}}{a^{2}}\left(\frac{a^{\prime \prime \prime \prime}}{a}+4 \frac{a^{\prime \prime \prime} a^{\prime}}{a^{2}}+\frac{3 a^{\prime \prime}{ }^{2}}{a^{2}}\right)\right. \\
& +\frac{m^{6} a^{6}}{32 \omega^{9}}\left(\frac{28 a^{\prime \prime \prime} a^{\prime}}{a^{2}}+\frac{126 a^{\prime \prime} a^{\prime 2}}{a^{3}}+21 \frac{a^{\prime \prime 2}}{a^{2}}+21 \frac{a^{\prime 4}}{a^{4}}\right) \\
& -\frac{231 m^{8} a^{8}}{32 \omega^{11}}\left(\frac{a^{\prime \prime} a^{\prime 2}}{a^{3}}+\frac{a^{\prime 4}}{a^{4}}\right)+\frac{1155 m^{10} a^{10}}{128 \omega^{13}} \frac{a^{\prime 4}}{a^{4}} \\
& +\left(\xi-\frac{1}{6}\right)\left[-\frac{6}{\omega}\left(\frac{a^{\prime \prime}}{a}-\frac{a^{\prime 2}}{a^{2}}\right)-\frac{3 m^{2} a^{2}}{\omega^{3}}\left(\frac{2 a^{\prime \prime}}{a}-\frac{a^{\prime 2}}{a^{2}}\right)\right. \\
& +\frac{9 m^{4} a^{4} a^{\prime 2}}{\omega^{5}}+\frac{3 m^{2} a^{2}}{2 \omega^{5}}\left(\frac{a^{\prime \prime \prime \prime}}{a}-\frac{2 a^{\prime \prime} a^{\prime 2}}{a^{3}}+\frac{a^{\prime 4}}{a^{4}}\right) \\
& -\frac{15 m^{4} a^{4}}{4 \omega^{7}}\left(\frac{4 a^{\prime \prime \prime} a^{\prime}}{a^{2}}+\frac{3 a^{\prime \prime}}{a^{2}}+\frac{8 a^{\prime \prime} a^{\prime 2}}{a^{3}}-\frac{a^{\prime 4}}{a^{4}}\right) \\
& \left.+\frac{105 m^{6} a^{6}}{8 \omega^{9}}\left(\frac{8 a^{\prime \prime} a^{\prime 2}}{a^{3}}+\frac{5 a^{\prime 4}}{a^{4}}\right)-\frac{945 m^{8} a^{8}}{8 \omega^{\prime 11}} \frac{a^{\prime 4}}{a^{4}}\right] \\
& +\left(\xi-\frac{1}{6}\right)^{2}\left[-\frac{9}{\omega^{3}}\left(\frac{a^{\prime \prime \prime \prime}}{a}-\frac{4 a^{\prime \prime \prime} a^{\prime}}{a^{2}}-\frac{3 a^{\prime \prime}}{a^{2}}+\frac{6 a^{\prime \prime} a^{\prime 2}}{a^{3}}\right)\right. \\
& \left.\left.+\frac{27 m^{2} a^{2}}{2 \omega^{5}}\left(\frac{4 a^{\prime \prime \prime} a^{\prime}}{a^{2}}+\frac{3 a^{\prime \prime 2}}{a^{2}}-\frac{6 a^{\prime \prime} a^{\prime 2}}{a^{3}}\right)-\frac{135 m^{4} a^{4}}{\omega^{7}} \frac{a^{\prime \prime} a^{\prime 2}}{a^{3}}\right]\right] .
\end{aligned}
$$

The formulae (C.1)-(C.8) are valid for a general flat RW spacetime.

In de Sitter space,

$$
\begin{gathered}
\left(\frac{2 a^{\prime \prime \prime} a^{\prime}}{a^{2}}-\frac{a^{\prime \prime 2}}{a^{2}}-\frac{4 a^{\prime \prime} a^{\prime 2}}{a^{3}}\right)=0, \\
\left(\frac{2 a^{\prime \prime \prime \prime}}{a}-\frac{10 a^{\prime \prime \prime} a^{\prime}}{a^{2}}-\frac{5 a^{\prime \prime 2}}{a^{2}}+\frac{16 a^{\prime \prime} a^{\prime 2}}{a^{3}}\right)=0, \\
\left(\frac{a^{\prime \prime \prime \prime}}{a}-\frac{4 a^{\prime \prime \prime} a^{\prime}}{a^{2}}-\frac{3 a^{\prime \prime 2}}{a^{2}}+\frac{6 a^{\prime \prime} a^{\prime 2}}{a^{3}}\right)=0,
\end{gathered}
$$

so that the $\left(\xi-\frac{1}{6}\right)^{2} / \omega^{3}$ terms in $\rho_{k A 4}, p_{k A 4}$ and $\left\langle T^{\mu}{ }_{\mu}\right\rangle_{k A 4}$ vanish. This will simplify calculations in Sections 3, 4, 5 to some extent.

In the following we check that the four-divergence of the subtraction terms is zero, at each adiabatic order. This is actually implied by the construction of WKB mode $v_{k}^{(n)}$ in (B.1) which satisfies the equation (B.2), ie, $v_{k}^{(n)}$ satisfies the field equation (5) to the $n$th order. We still give explicit calculations as a checking in the following. By (C.1) (C.2), the four-divergence of the 0th-order subtraction terms is zero,

$$
\begin{aligned}
\left\langle T_{; \nu}^{0 \nu}\right\rangle_{k A 0} & =\rho_{k A 0}^{\prime}+3 \frac{a^{\prime}}{a}\left(\rho_{k A 0}+p_{k A 0}\right) \\
& =\frac{k^{3}}{4 \pi^{2}}\left(-\frac{a^{\prime}\left(3 m^{2} a^{2}+4 k^{2}\right)}{a^{5} \omega}+\frac{a^{\prime}\left(3 m^{2} a^{2}+4 k^{2}\right)}{a^{5} \omega}\right)=0 .
\end{aligned}
$$


Thus, the 0th-order regularized spectral stress tensor respects the covariant conservation

$$
\left\langle T_{; \nu}^{\mu \nu(0)}\right\rangle_{k r e g}=\left\langle T_{; \nu}^{\mu \nu}\right\rangle_{k}-\left\langle T_{; \nu}^{\mu \nu}\right\rangle_{k A 0}=0
$$

which holds for a general coupling $\xi$ and a mass $m$. For the scalar field considered in this paper, the $k$-modes of stress tensor are independent of each other, so, after $k$-integration of the spectral stress tensor, the regularized stress tensor also respects the covariant conservation.

Similarly, from (C.4) (C.5) for the 2nd-order, by explicit calculation,

$$
\begin{aligned}
\rho_{k A 2}^{\prime}= & \frac{k^{3}}{4 \pi^{2} a^{4}}\left[\left(\frac{m^{2} a a^{\prime}}{\omega}+\frac{m^{4} a^{2} a^{\prime} a^{\prime \prime}+m^{4} a\left(a^{\prime}\right)^{3}}{4 \omega^{5 / 2}}-\frac{5 m^{6} a^{3}\left(a^{\prime}\right)^{3}}{8 \omega^{7 / 2}}\right)\right. \\
& +\left(\xi-\frac{1}{6}\right)\left(\frac{6 a^{\prime 3}-6 a a^{\prime} a^{\prime \prime}}{a^{3} \omega}+\frac{3 m^{2}\left(a^{\prime}\right)^{3}-6 m^{2} a a^{\prime} a^{\prime \prime}}{a \omega^{3 / 2}}+\frac{9 m^{4} a\left(a^{\prime}\right)^{3}}{\omega^{5 / 2}}\right) \\
& \left.-\frac{4 a^{\prime}}{a}\left(\omega-\left(\xi-\frac{1}{6}\right)\left(\frac{3 a^{\prime 2}}{\omega a^{2}}+\frac{3 m^{2} a^{\prime 2}}{\omega^{3 / 2}}\right)+\frac{m^{4} a^{2} a^{\prime 2}}{8 \omega^{5 / 2}}\right)\right], \\
& 3 \frac{a^{\prime}}{a}\left(\rho_{k A 2}+p_{k, A 2}\right)=- \text { r.h.s. of }(C .14),
\end{aligned}
$$

so the four divergence of the 2 nd order subtraction term is zero,

$$
\rho_{k A 2}^{\prime}+3 \frac{a^{\prime}}{a}\left(\rho_{k A 2}+p_{k, A 2}\right)=0
$$

and the covariant conservation is respected by the 2nd-order regularized stress tensor,

$$
\left\langle T_{; \nu}^{\mu \nu(2)}\right\rangle_{r e g}=\left\langle T_{; \nu}^{\mu \nu}\right\rangle-\left\langle T_{; \nu}^{\mu \nu}\right\rangle_{A 2}=0 .
$$

Finally, from (C.6) and (C.7) it is checked that the four-divergence of the 4th order subtraction term is zero,

$$
\rho_{k A 4}^{\prime}+3 \frac{a^{\prime}}{a}\left(\rho_{k A 4}+p_{k, A 4}\right)=0
$$

(the explicit expressions of the above two terms are lengthy and we do not list here,) so the covariant conservation is respected by the 4th-order regularized stress tensor,

$$
\left\langle T_{; \nu}^{\mu \nu(4)}\right\rangle_{r e g}=\left\langle T_{; \nu}^{\mu \nu}\right\rangle-\left\langle T_{; \nu}^{\mu \nu}\right\rangle_{A 4}=0 .
$$

The covariant conservations (C.13) (C.16) (C.18) are valid for a general flat RW spacetime. 